\title{
The Inverse Problem in Classical Statistical Mechanics
}

\author{
J. T. Chayes ${ }^{1, \star, \dagger}$, L. Chayes ${ }^{1, \star \star, \dagger}$, and Elliott H. Lieb ${ }^{2, \star \star}$ \\ 1 Department of Physics, Princeton University, Princeton, NJ 08544, USA \\ 2 Departments of Mathematics and Physics, Princeton University, Princeton, NJ 08544, USA
}

\begin{abstract}
We address the problem of whether there exists an external potential corresponding to a given equilibrium single particle density of a classical system. Results are established for both the canonical and grand canonical distributions. It is shown that for essentially all systems without hard core interactions, there is a unique external potential which produces any given density. The external potential is shown to be a continuous function of the density and, in certain cases, it is shown to be differentiable. As a consequence of the differentiability of the inverse map (which is established without reference to the hard core structure in the grand canonical ensemble), we prove the existence of the Ornstein-Zernike direct correlation function. A set of necessary, but not sufficient conditions for the solution of the inverse problem in systems with hard core interactions is derived.
\end{abstract}

\section{Introduction}

The inverse problem in classical statistical mechanics concerns the relationship between classical systems and their equilibrium single particle densities. Consider a finite temperature classical system of $N$ particles characterized by an interaction $W\left(x_{1}, \ldots, x_{N}\right)$, where $x_{i}$ denotes all the coordinates (e.g. space, spin, etc.) of the $i^{\text {th }}$ particle. $W$ is henceforth regarded as a fixed, given function. It need not be symmetric. If an external potential, $U(x)$, is applied to the system, the density of the $i^{\text {th }}$ particle in the canonical distribution is

$$
\varrho_{i}\left(U ; x_{i}\right)=Z_{U}^{-1} \int \exp \left[-W\left(x_{1}, \ldots, x_{N}\right)-\sum_{j=1}^{N} U\left(x_{j}\right)\right] d x_{1} \ldots \widehat{d x}_{i} \ldots d x_{N},
$$

where $\widehat{d x}_{i}$ indicates that there is no integration over $x_{i}$, and

* Work partially supported by NSF grant PHY-8117463

$\star \star$ Work partially supported by NSF grant PHY-8116101 A01

${ }^{\dagger}$ Address beginning September 1983: Department of Physics, Harvard University, Cambridge, MA 02138, USA 


$$
Z_{U}=\int \exp \left[-W\left(x_{1}, \ldots, x_{N}\right)-\sum_{i=1}^{N} U\left(x_{i}\right)\right] d x_{1} \ldots d x_{N}
$$

is the canonical partition function for the external potential $U$. For convenience, we have taken inverse temperature $\beta=1$. The equilibrium single particle density of the system is then simply

$$
\varrho(U ; x)=\sum_{i=1}^{N} \varrho_{i}(U ; x) .
$$

Here we address the inverse problem: Given a function $\varrho(x) \geqq 0$ with $\int \varrho(x) d x=N$, does there exist a single particle external potential, $U(x)$, such that $\varrho(x)$ is the equilibrium density of the system with potential $U(x)$ as given by (1.1)-(1.3)?

One of us [1] (E.H.L.) has studied an analogous quantum mechanical problem in which $\varrho(x)$ is the one particle reduction of a pure ground state $\Psi\left(x_{1}, \ldots, x_{N}\right)$. There it was shown that there exist densities $\varrho(x)$ which do not correspond to the pure ground state of a system with potential $W+\Sigma U$ for any choice of $U$. Levy [2] reached the same conclusion. Englisch and Englisch [3] proved that even if mixed ground states are allowed, one can construct a $\varrho$ which does not come from any $U$. A similar problem for quantum lattice systems has recently been investigated by Hugenholtz [4]. However, rigorous results on the inverse conjecture for classical statistical mechanics have been limited. Nevertheless, the classical conjecture is implicit in many theories of non-uniform fluids; in particular, certain models of the liquid-vapor interface depend crucially on the validity of the conjecture for specific systems (see Evans [5] and references therein). In this context, several authors have derived formal expressions for the potential for the case of hard core interactions in one dimension [6-8].

In this paper, we provide a proof of the inverse conjecture for a large class of systems in both the canonical and the grand canonical distributions. For a single species of particles in the canonical distribution, we prove that if

$$
\int W_{+}\left(x_{1}, \ldots, x_{N}\right) \prod_{i=1}^{N} \varrho\left(x_{i}\right) d^{N} x<\infty,
$$

and

$$
\int \exp \left[-W\left(x_{1}, \ldots, x_{N}\right)\right] \prod_{i=1}^{N} \varrho\left(x_{i}\right) d^{N} x<\infty,
$$

then there exists a unique potential $U(x)$ corresponding to the density $\varrho(x)$. In expression (1.4), $W_{+}$denotes the positive part of the interaction potential $W$, namely $W_{+}\left(x_{1}, \ldots, x_{N}\right)=\max \left\{W\left(x_{1}, \ldots, x_{N}\right), 0\right\}$.

It is worth pointing out that condition (1.4) is by no means superfluous. Indeed, there are numerous examples of systems that violate (1.4) in which the inverse conjecture fails. For instance, if $W$ is a sum of two-body hard core potentials of radius $R$, and $\varrho(x)$ is an integrable function supported entirely within a region of radius $R$, then clearly there does not exist any well defined physical potential $U(x)$ such that $\varrho(x)=\varrho(U ; x)$. Condition (1.4) excludes systems of this sort, but it allows 
$W$ to have integrable singularities. Condition (1.5) essentially excludes systems that would have infinite free energy if the single particle density were $\varrho(x)$. The physical reasoning underlying this condition is explained in more detail in Sect. 2.

We shall always assume that the coordinates, $x_{i}$, of the $i^{\text {th }}$ particle lie in some $\sigma$-finite measure space $\langle\Lambda, d x\rangle$. The assumption of $\sigma$-finiteness is technically necessary to guarantee that the product space $\left\langle\Lambda^{N}, d^{N} x\right\rangle$ is well defined and obeys the standard product space theorems (e.g., Fubini's theorem). All spaces of physical interest are $\sigma$-finite. We emphasize that we place no other restrictions on the measure space $\langle\Lambda, d x\rangle$. The generality of the measure space $\langle\Lambda, d x\rangle$ is not simply a mathematical nicety, for it allows our results to be applied to a very large class of physical systems. In particular, $\langle\Lambda, d x\rangle$ may represent a single particle space for a system in any dimension. The space $\langle\Lambda, d x\rangle$ need not be $\mathbb{R}^{n}$; it may, for example, be the surface of a sphere or a torus, or even a classical lattice. Moreover, $\Lambda$ need not correspond exclusively to spatial variables - the results apply equally well to momentum space, phase space or any space of internal variables (e.g., intrinsic spin).

Among the continuous systems to which our results apply, one of notable physical interest is the Coulomb gas in two dimensions (at temperatures for which the system exists). Regarding $\langle\Lambda, d x\rangle$ as a space of spin variables, one may of course apply these results to Ising or Heisenberg type magnets. Finally, we remark that if one wishes to consider densities defined on some subset of the natural space of a system, it is possible to simply integrate out the "unnecessary" degrees of freedom and take $\langle\Lambda, d x\rangle$ to be the subspace on which the densities are defined.

We also stress that no specific assumptions have been made on the form of the interaction $W\left(x_{1}, \ldots, x_{N}\right)$. In general, $W$ will be a sum of one-body through $N$-body interactions, none of which are endowed with any special symmetry.

Existence and uniqueness of the potential $U$ corresponding to a density $\varrho$ are proved in Sect. 2. Next we examine some properties of the inverse map $U(\varrho)$. In Sect. 3 , it is shown that the inverse map is continuous in a sense which is natural for the system. Differentiability of the inverse map is discussed in Sect. 4. There we prove that if $W$ is a bounded interaction, then the inverse map possesses a Fréchet derivative and we show that this derivative provides some local information on the structure of $U$.

Natural generalizations of the inverse problem are discussed in Sects. 5 and 6. The inverse problem for systems with several species of particles is investigated in Sect. 5 , in which it is shown that there exist unique families of potentials that produce a given family of densities of various particle species. In Sect. 6, we study systems with massive or charged coupling. For these systems, we prove that there exists a unique gravitational or electric potential corresponding to a given mass or charge density.

The inverse problem may also be formulated within the context of the grand canonical ensemble. This is done in Sect. 8. The system is characterized by an infinite family, $\left(W_{N}\right), N=1,2, \ldots$, of interaction potentials. Suppose that we are given a density function $\varrho(x) \geqq 0$ with $\int \varrho(x) d x=n$, where $n$ is the expected particle number of the system. We assume that a stability condition analogous to Eq. (1.5) is satisfied and that a few of the $W_{N}$ satisfy (1.4). Under these assumptions, it is shown (Subsect. 8ii) that there exists a unique external potential $U(x)$ such that 
$\varrho(x)$ is the single particle density of a system with total potentials $\left(W_{N}\left(x_{1}, \ldots, x_{N}\right)\right.$ $\left.+\sum_{i=1}^{N} U\left(x_{i}\right)\right)$. It is worth noting that only one interaction, $W_{\tilde{N}}$ for $\tilde{N}>n$, is required to obey condition (1.4). Hence, these results are applicable to systems in which the grand canonical partition function has been truncated by taking $W_{N} \equiv \infty$ for all $N$ greater than some fixed $N^{\prime} \geqq \tilde{N}$.

In Subsects. 8(iii) and 8(iv), we prove continuity and differentiability of the inverse map $U(\varrho)$ for the grand canonical ensemble. The methods of Subsect. 8(iv) also provide us with a local existence result for hard core interactions. The inverse problem for several species in the grand canonical ensemble is discussed in Subsect. 8(v). In 8(vi), systems with massive or charged coupling are investigated. Within the context of the grand canonical ensemble, there are various forms of the inverse problem for these systems. If one may only adjust the overall chemical potential, it is shown that there exists an electric potential which produces a given charge density and total particle number. However, the ability to adjust the chemical potentials of particles of different charge independently implies that we can also find an electric potential and a set of fugacities corresponding to a given charge density with a preprescribed set of expected particle numbers.

We have already remarked that the inverse conjecture fails for some systems with hard core potentials. A more detailed discussion of restrictions in the hard core case appears in Sect. 9.

Some technical theorems on the convergence of product functions, used in proving many of the aforementioned results, have been relegated to an appendix.

\section{Existence and Uniqueness}

Let $\langle\Lambda, d x\rangle$ be a measure space and let $\left\langle\Lambda^{N}, d^{N} x\right\rangle$ denote the $N$-fold cartesian product measure space. Let $X=\left(x_{1}, \ldots, x_{N}\right)$ denote an element of $\Lambda^{N}$. Given the function

$$
\varrho: \Lambda \rightarrow \mathbb{R}, \varrho(x) \geqq 0, \int_{\Lambda} \varrho(x) d x=N,
$$

it is convenient to introduce the normalized measure

$$
d \mu=\frac{1}{N} \varrho(x) d x
$$

on $\Lambda$ and the corresponding product measure

$$
d^{N} \mu=\prod_{i=1}^{N}\left[\varrho\left(x_{i}\right) / N\right] d^{N} x
$$

on $\Lambda^{N}$.

Notation. A function $f: M \rightarrow \mathbb{R}$ defined on a measure space $\langle M, d m\rangle$ is said to be in $L^{p}(d m)$ if $\left[\int|f(m)|^{p} d m\right]^{1 / p} \equiv\|f\|_{p(d m)}$ is finite. $\|f\|_{\infty} \equiv \operatorname{ess} \sup |f(m)|$.

Let $W(X)$, the interaction, be a given function which satisfies

$$
W: \Lambda^{N} \rightarrow \mathbb{R}, W_{+} \in L^{1}\left(d^{N} \mu\right), e^{-W} \in L^{1}\left(d^{N} \mu\right) .
$$


Then we can introduce yet another finite measure

$$
d \lambda=e^{-W} d^{N} \mu
$$

on $\Lambda^{N}$. The condition $e^{-W} \in L^{1}\left(d^{N} \mu\right)$ insures that $d \lambda$ is absolutely continuous ${ }^{1}$ with respect to $d^{N} \mu$, while $W_{+} \in L^{1}\left(d^{N} \mu\right)$ implies that $d^{N} \mu$ is absolutely continuous with respect to $d \lambda$. Thus $d^{N} \mu$ and $d \lambda$ are equivalent ${ }^{1}$ measures on $\Lambda^{N}$.

We should like to determine whether there exists a function $U: \Lambda \rightarrow \mathbb{R}$ such that $\varrho=\varrho(U)$ a.e. $[d x]$, where $\varrho(U)$ is given by Eq. (1.3). Actually, we note $U$ only appears in the definition of $\varrho(U)$ in the form $e^{-U}$. Since it may be the case that $e^{-U}=0$ (which would correspond to $U=\infty$ ) on sets of nonzero measure, let us instead determine whether there exists a function $e^{-U}: \Lambda \rightarrow \mathbb{R}^{+}$such that $\varrho=\varrho(U)$ a.e. $[d x]$.

Alternatively, let us consider the canonical density of a system at external potential

$$
U=-\log (\varrho / N)+V,
$$

where $\varrho$ is the given function in Eq. (2.1). In terms of the function $V$, the canonical density of the $i^{\text {th }}$ particle is

$$
\begin{aligned}
\varrho_{V, i}\left(x_{i}\right)= & {[Z(V)]^{-1} \int \exp \left[-W\left(x_{1}, \ldots, x_{N}\right)-\sum_{j=1}^{N} V\left(x_{j}\right)\right] } \\
& \cdot \prod_{j=1}^{N}\left[\varrho\left(x_{j}\right) / N\right] d x_{1} \ldots \widehat{d x}_{i} \ldots d x_{N},
\end{aligned}
$$

where

$$
Z(V)=\int \exp \left[-W\left(x_{1}, \ldots, x_{N}\right)-\sum_{i=1}^{N} V\left(x_{i}\right)\right] d^{N} \mu .
$$

Then the single particle density as a function of $V$ is

$$
\varrho_{V}(x)=\sum_{i=1}^{N} \varrho_{V, i}(x) \text {. }
$$

The inverse problem in terms of the potential $U$ is clearly equivalent to the problem of finding a function $V: \Lambda \rightarrow \mathbb{R}$, or $e^{-V}: \Lambda \rightarrow \mathbb{R}^{+}$, such that $\varrho=\varrho_{V}$ a.e. [dx].

Note. We emphasize that $U$ is the physical external potential. $V$ is a useful mathematical construct which we shall call a potential in the hope that such terminology will not be confusing to the reader.

Remark. The decomposition in Eq. (2.6) is useful in the sense that it divides out the ideal gas $(W \equiv 0)$ behavior. Whenever $W \equiv 0$, the inverse problem is trivial; the solution is simply $V=0$. Moreover, in the general case, $\varrho$ need not have support on all of $\Lambda$. Hence the external potential, $U$, may be required to provide "walls" which cut-off the density in part of the system. The piece of the potential which accomplishes this is $-\log (\varrho / N)$, whereas $V$ only alters the density on the support of $\varrho$.

1 Refer to the appendix for definitions of absolute continuity and equivalence of measures 
The existence of a $V$ such that $\varrho=\varrho_{V}$ is established by means of a variational technique. For fixed $W$ and $\varrho$, consider the functional

$$
\mathfrak{F}(V)=[Z(V)]^{-1} \exp \left[-N \int V d \mu\right] \text {. }
$$

To insure that the numerator and denominator of $\mathfrak{F}$ are separately well defined, we restrict attention to the set of functions $\mathscr{V}$ defined by

$$
\mathscr{V}=\left\{V: \Lambda \rightarrow \mathbb{R} \mid V \in L^{1}(d \mu), Z(V)<\infty\right\} .
$$

Remark. (i) The set $\mathscr{V}$ is not empty. In particular $0 \in \mathscr{V}$ since $0 \in L^{1}(d \mu)$, and $e^{-W} \in L^{1}\left(d^{N} \mu\right) \Rightarrow Z(0)<\infty$.

(ii) The condition $e^{-W} \in L^{1}\left(d^{N} \mu\right)$ can be replaced by the condition $\mathscr{V} \neq \emptyset$, since if there exists $\tilde{V} \in \mathscr{V}$, then by taking $\tilde{W}=W+\sum_{i=1}^{N} \tilde{V}\left(x_{i}\right)$, we have an equivalent problem for the system characterized by interaction $\tilde{W}$.

(iii) We observe that $Z(V)>0$ for all $V$ in $\mathscr{V}$ since, by Jensen's inequality,

$$
Z(V) \geqq \exp \left[-\left\|W_{+}\right\|_{1\left(d^{N} \mu\right)}-N \int V d \mu\right]>0 .
$$

Formally, the variational derivative of $\log \mathfrak{F}$ is given by

$$
\frac{\delta \log \mathfrak{F}}{\delta V(x)}=\varrho_{V}(x)-\varrho(x) .
$$

Thus the problem of finding a potential corresponding to $\varrho$ may be reduced to that of showing the existence of an extremum (in $\mathscr{V}$ ) of $\mathfrak{F}$.

From (2.10) and (2.12), we have:

Proposition 2.1. Suppose $W_{+} \in L^{1}\left(d^{N} \mu\right)$ and $e^{-W} \in L^{1}\left(d^{N} \mu\right)$. Then:

(a) $\mathfrak{F}$ is (uniformly) bounded above in $\mathscr{V}$.

$$
\mathfrak{F}(V) \leqq \exp \left[\left\|W_{+}\right\|_{1\left(d^{N} \mu\right)}\right],
$$

(b) For all $C \in \mathbb{R}$ and $V \in \mathscr{V}, \mathfrak{F}(V+C)=\mathfrak{F}(V)$.

Let us define

$$
R=\sup _{V \in \mathscr{V}} \mathfrak{F}(V)<\infty
$$

Theorem 2.2. Under the assumptions of Proposition 2.1, there exists $V \in \mathscr{V}$ which maximizes $\mathfrak{F}$.

Proof. Let $\left(V_{n}\right)$ be a maximizing sequence in $\mathscr{V}$ for $\mathfrak{F}$, i.e. $\lim _{n \rightarrow \infty} \mathfrak{F}\left(V_{n}\right)=R$. By Proposition 2.1(b), without loss of generality we may add a constant to each $V_{n}$ so that $Z\left(V_{n}\right)=1$.

With this normalization, the sequence $\left(F_{n}\right)$ of functions defined by

$$
F_{n}=\prod_{i=1}^{N} \exp \left[-V_{n}\left(x_{i}\right) / 2\right]
$$

satisfies $F_{n} \in L^{2}(d \lambda)$ with $\left\|F_{n}\right\|_{2(d \lambda)}=1$. Thus, by the Banach-Alaoglu theorem, there is a subsequence [also denoted by $\left.\left(F_{n}\right)\right]$ which converges weakly in $L^{2}(d \lambda)$, i.e. 
$F_{n} \rightarrow F$ in $L^{2}(d \lambda)$. Since $d \lambda$ is equivalent to the product measure $d^{N} \mu$ on $\Lambda^{N}$, it follows from Theorem A.3 that $F$ is a product of identical, $[d \mu]$ measurable functions. Hence there exists $V: \Lambda \rightarrow \mathbb{R}$ such that

$$
F=\prod_{i=1}^{N} \exp \left[-V\left(x_{i}\right) / 2\right] .
$$

We shall show that $V \in \mathscr{V}$ and that $V$ maximizes $\mathfrak{F}$.

First we consider the denominator of $\mathfrak{F}$. Since the denominator is weakly lower semicontinuous in $F_{n}$,

$$
1=\lim _{n \rightarrow \infty} Z\left(V_{n}\right)=\lim _{n \rightarrow \infty} \int d \lambda F_{n}^{2} \geqq \int d \lambda F^{2}=Z(V) .
$$

Thus $Z(V)$ is finite. In order to establish that $V \in \mathscr{V}$, it suffices to show that $V \in L^{1}(d \mu)$.

We first show that $V_{-} \in L^{1}(d \mu)$, where $V_{-}$denotes the negative part of the function $V=V_{+}-V_{-}$. Let $T=\left\{x \in \Lambda \mid V_{-}(x)>0\right\}$. If $\mu(T)=0$, then clearly $V_{-} \in L^{1}(d \mu)$. Suppose $\mu(T)>0$. Then [since $\left.\int e^{h} d m<\infty \Rightarrow h_{+} \in L^{1}(d m)\right]$, the finiteness of $Z(V)$ implies that $\left(-W-\sum V\right)_{+} \in L^{1}\left(d^{N} \mu\right)$. But

$$
\left(-W-\sum V\right)_{+} \geqq\left(-\sum V\right)_{+}-W_{+},
$$

and $W_{+} \in L^{1}\left(d^{N} \mu\right)$. Hence $\left(-\sum V\right)_{+} \in L^{1}\left(d^{N} \mu\right)$. Moreover

$$
\left[-\sum_{i=1}^{N} V\left(x_{i}\right)\right]_{+} \geqq \prod_{i=1}^{N} \chi_{T}\left(x_{i}\right) \sum_{i=1}^{N} V_{-}\left(x_{i}\right)
$$

where $\chi_{T}$ is the characteristic function of the set $T$. Then, since $\int \chi_{T} d \mu=\mu(T)>0$, it is clear that $V_{-} \in L^{1}(d \mu)$.

In order to show that $V_{+} \in L^{1}(d \mu)$, let us define the truncated functions

$$
W^{m}(X)=\left\{\begin{array}{ll}
W(X) & W(X) \leqq m \\
m & W(X)>m
\end{array},\right.
$$

and

$$
V^{k}(x)= \begin{cases}V(x) & V(x) \leqq k \\ k & V(x)>k\end{cases}
$$

where $m$ and $k$ are positive integers. $V_{-} \in L^{1}(d \mu) \Rightarrow V^{k} \in L^{1}(d \mu)$. By Jensen's inequality

$$
\begin{aligned}
& \exp \left[-\frac{N}{2} \int\left(V_{n}-V^{k}\right) d \mu-\int\left(W-W^{m}\right) d^{N} \mu\right] \\
& \quad \leqq \int\left[\prod^{N} \exp \left(-V_{n} / 2\right) \exp \left(+V^{k} / 2\right)\right] \exp \left(W^{m}\right) d \lambda .
\end{aligned}
$$

We note that $\exp \left(W^{m}\right) \in L^{\infty}(d \lambda)$ and $\prod \exp \left(V^{k} / 2\right) \in L^{\infty}(d \lambda)$, and that $L^{\infty}(d \lambda) \subset L^{2}(d \lambda)$, since the measure $d \lambda$ is finite. However, by the convergence of $\left(F_{n}\right)$, and the fact $\int V_{n} d \mu \rightarrow \frac{1}{N} \log \frac{1}{R}$, we have, for all $m$ and $k$, 


$$
\begin{aligned}
& \lim _{n \rightarrow \infty} \exp \left[-\frac{N}{2} \int\left(V_{n}-V^{k}\right) d \mu-\int\left(W-W^{m}\right) d^{N} \mu\right] \\
& \quad \leqq \int\left[\prod^{N} \exp \left[-\left(V-V^{k}\right) / 2\right]\right] \exp \left[-\left(W-W^{m}\right)\right] d^{N} \mu \leqq 1 .
\end{aligned}
$$

The second inequality follows from the fact that $V^{k} \leqq V$ and $W^{m} \leqq W$. By dominated convergence, we may remove the truncation on $W$, i.e.

$$
\lim _{m \rightarrow \infty} \int\left(W-W^{m}\right) d^{N} \mu=0 .
$$

Then, recalling that $V_{-} \in L^{1}(d \mu)$, we obtain from (2.24)

$$
\lim _{n \rightarrow \infty} \exp \left(-N \int V_{n} d \mu\right) \leqq \exp \left[N\left(\int V_{-} d \mu-\int V_{+}^{k} d \mu\right)\right],
$$

where

$$
V_{+}^{k}(x)=\left\{\begin{array}{ll}
V_{+}(x) & V_{+}(x) \leqq k \\
k & V_{+}(x)>k
\end{array} .\right.
$$

By monotone convergence of $V_{+}^{k}$,

$$
\lim _{n \rightarrow \infty} \exp \left(-N \int V_{n} d \mu\right) \leqq \exp \left[N\left(\int V_{-} d \mu-\int V_{+} d \mu\right)\right]
$$

Recall that for all $n, Z\left(V_{n}\right)=1$ and hence $\mathfrak{F}\left(V_{n}\right)=\exp \left(-N \int V_{n} d \mu\right)$. Then by Eq. (2.28)

$$
\exp \left[N\left(\int V_{-} d \mu-\int V_{+} d \mu\right)\right] \geqq \lim _{n \rightarrow \infty} \mathfrak{F}\left(V_{n}\right)=R>0 .
$$

Evidently $V_{+} \in L^{1}(d \mu)$, and hence $V \in \mathscr{V}$.

Finally, to see that $V$ maximizes $\mathfrak{F}$, recall that by Eq. $(2.18) Z(V) \leqq 1$. Combining this with Eq. (2.29), we find

$$
\mathfrak{F}(V) \geqq R .
$$

Noting that $R=\sup _{s \in \mathscr{V}} \mathfrak{F}(s)$ and $V \in \mathscr{V}$, we obtain the desired result.

Corollary. If $\left(V_{n} \in \mathscr{V}\right)$ is a maximizing sequence for $\mathfrak{F}$ normalized so that $Z\left(V_{n}\right)=1$, then some subsequence of the sequence $\left(F_{n}\right)$, defined by Eq. (2.16), converges strongly in $L^{2}(d \lambda)$.

Proof. We recall that if $Z\left(V_{n}\right)=1$, then some subsequence of $\left(F_{n}\right)$ converges weakly in $L^{2}(d \lambda)$. Thus, in order to establish strong convergence, it suffices to show that the norms converge, i.e. $Z\left(V_{n}\right) \rightarrow Z(V)$. By Eq. (2.18), $\lim _{n \rightarrow \infty} Z\left(V_{n}\right) \geqq Z(V)$. Suppose $\lim _{n \rightarrow \infty} Z\left(V_{n}\right)>Z(V)$. Then it follows from Eq. (2.28) that

$$
\mathfrak{F}(V)=[Z(V)]^{-1} \exp \left[-N \int V d \mu\right]>\lim _{n \rightarrow \infty} \mathfrak{F}\left(V_{n}\right)=R,
$$

a contradiction. Thus $F_{n} \rightarrow F$ strongly in $L^{2}(d \lambda)$.

Remark. It should be pointed out that the convergence of $\left(F_{n}\right)$ to a product function could have been established by relying on specific properties of the 
maximizing sequence (cf. proof of Theorem 6.2), rather than by invoking Theorem A.3. However, for the proof of Theorem 2.2, these methods would have been unnecessarily cumbersome.

Remark. Had the maximizing $V$ satisfied $e^{-V} \in L^{1}(d \mu)$, the proof of Theorem 2.2 could have been simplified. One could have taken a maximizing sequence which converged in a single particle space and considerations about the convergence of product functions would have been unnecessary. Theorem 2.2 demonstrates that the maximizing $V$ is in $\mathscr{V}$, and hence it is natural to ask whether it is also in the space

$$
\tilde{\mathscr{V}}=\left\{V: \Lambda \rightarrow \mathbb{R} \mid V \in L^{1}(d \mu), e^{-V} \in L^{1}(d \mu)\right\} .
$$

As the following example demonstrates, generically $V \notin \tilde{\mathscr{V}}$. Evidently, it is the fully interacting measure, $d \lambda$, and not the single particle space measure, $d \mu$, which is of physical relevance.

Example. Consider a two particle system in one dimension on the unit interval, $\Lambda=(0,1)$, with interaction potential $W(x, y)=(x y)^{-1 / 2}$. A single particle density of the form

$$
\varrho(x)=Z^{-1} \frac{2}{x} \int_{0}^{x} \exp [-1 / \sqrt{\xi}] \frac{1}{\xi} \mathrm{d} \xi
$$

with

$$
Z=\int \exp [-1 / \sqrt{x y}] \frac{1}{x y} d x d y,
$$

may be produced by applying an external potential $U(x)=\log x$. It is easy to verify that $\varrho \in L^{1}(d x), W_{+} \in L^{1}\left(d^{2} \mu\right)$, and $e^{-W} \in L^{1}\left(d^{2} \mu\right)$. Thus $\varrho$ is an admissible density for this system. Hence by Theorem 2.2 there exists a $V \in \mathscr{V}$ which maximizes $\mathfrak{F}$. Indeed, $V$ is given by $V=\log (x \varrho / 2)$.

Clearly $Z(V)=Z<\infty$. Moreover, we easily verify that $\int_{0}^{1}|V| \varrho d x<\infty$. Thus $V \in L^{1}(d \mu)$, so that $V \in \mathscr{V}$. However, $e^{-V} \notin L^{1}(d \mu)$, and hence $V \notin \tilde{\mathscr{V}}$ since

$$
\int_{0}^{1} e^{-V} d \mu=\int_{0}^{1} \frac{1}{x} d x=\infty
$$

Theorem 2.3. Under the assumptions of Proposition 2.1, there exists $V \in \mathscr{V}$ such that $\varrho(x)=\varrho_{V}(x)$ for all $x \in \Lambda$.

Remark. Below we shall prove that there exists a $V \in \mathscr{V}$ such that $\varrho=\varrho_{V}$ a.e. $[d x]$ on $\Lambda$. However, by the definition of $\varrho_{V}$ [Eq. (2.7)-(2.9)], it is clear that if we find a $V \in \mathscr{V}$ such that $\varrho=\varrho_{V}$ a.e. $[d x]$, then we can modify $V$ on a set of zero measure to obtain a $\tilde{V} \in \mathscr{V}$ such that $\varrho(x)=\varrho_{\tilde{V}}(x)$ for every $x \in \Lambda$.

Proof. By Theorem 2.2, there exists a $V \in \mathscr{V}$ such that $\mathfrak{F}(V)=R$. For this $V$, calculate $\varrho_{V}$ according to Eqs. (2.7)-(2.9) and define

$$
\eta(x)=\left\{\begin{array}{ll}
+1 & \varrho_{V}(x) \geqq \varrho(x) \\
-1 & \varrho_{V}(x)<\varrho(x)
\end{array} .\right.
$$


Note that $\eta \in L^{\infty}$, and hence for every $\varepsilon>0,(V+\varepsilon \eta) \in \mathscr{V} \Rightarrow \mathfrak{F}(V) \geqq \mathfrak{F}(V+\varepsilon \eta)$. Also

$$
\mathfrak{F}(V+\varepsilon \eta)=\mathfrak{F}(V)\left[1-\varepsilon \int\left(\varrho-\varrho_{V}\right) \eta d x\right]+O\left(\varepsilon^{2}\right) .
$$

Thus

$$
0 \leqq \lim _{\varepsilon \rightarrow 0} \varepsilon^{-1}[\mathfrak{F}(V)-\mathfrak{F}(V+\varepsilon \eta)]=\mathfrak{F}(V) \int\left(\varrho-\varrho_{V}\right) \eta d x=-\mathfrak{F}(V) \int \varrho-\varrho_{V} \mid d x
$$

Evidently $\varrho=\varrho_{V}$ a.e. $[d x]$.

Theorem 2.4. Under the assumptions of Proposition 2.1, if there exists a measurable function $V: \Lambda \rightarrow \mathbb{R}$ such that $\varrho=\varrho_{V}$ a.e. $[d x]$, then

(a) $V \in \mathscr{V}$.

(b) Up to a constant, $V$ is unique a.e. $[d \mu]$.

Proof. (a) If $\varrho=\varrho_{V}$ a.e. $[d x]$, then by definition $0<Z(V)<\infty$. Hence it suffices to show that $V \in L^{1}(d \mu)$. By a proof identical to that in Theorem 2.2 [cf. Eqs. (2.19) and (2.20)], $Z(V)<\infty \Rightarrow V_{-} \in L^{1}(d \mu)$.

Next we show that $V_{+} \in L^{1}(d \mu)$. Since $Z(V)>0$,

$$
\infty>Z(0) / Z(V)=[Z(V)]^{-1} \int e^{-W} \prod^{N} e^{-V} e^{+V} d^{N} \mu=\int \exp \left(\sum V\right) d \sigma_{V},
$$

where

$$
d \sigma_{V}=[Z(V)]^{-1}\left[e^{-W} \prod^{N} e^{-V} d^{N} \mu\right]
$$

is a unit measure. Thus $\left(\sum V\right)_{+} \in L^{1}\left(d \sigma_{V}\right)$. But

$$
\left(\sum V\right)_{+} \geqq \sum V=\sum V_{+}-\sum V_{-} .
$$

Moreover, since $V_{-} \in L^{1}(d \mu)$ and $\varrho_{V}=\varrho$,

$$
\infty>\int V_{-} \varrho d x=\int V_{-} \varrho_{V} d x=\int \sum V_{-} d \sigma_{V}
$$

$\Rightarrow \sum V_{-} \in L^{1}\left(d \sigma_{V}\right)$. Thus $\sum V_{+} \in L^{1}\left(d \sigma_{V}\right)$. Then

$$
\infty>\int \sum V_{+} d \sigma_{V}=\int V_{+} \varrho_{V} d x=\int V_{+} \varrho d x .
$$

(b) Suppose there exist $V_{1}$ and $V_{2}$ such that $\varrho_{V_{1}}=\varrho_{V_{2}}=\varrho$. By part (a), $V_{1}, V_{2} \in \mathscr{V}$. Then by Jensen's inequality

$$
Z\left(V_{1}\right) / Z\left(V_{2}\right) \geqq \exp \left[-\int \sum\left(V_{1}-V_{2}\right) d \sigma_{V_{2}}\right]=\exp \left[-N \int\left(V_{1}-V_{2}\right) d \mu\right],
$$

while

$$
Z\left(V_{2}\right) / Z\left(V_{1}\right) \geqq \exp \left[-\int \sum\left(V_{2}-V_{1}\right) d \sigma_{V_{1}}\right]=\exp \left[-N \int\left(V_{2}-V_{1}\right) d \mu\right] .
$$

Therefore

$$
Z\left(V_{1}\right) / Z\left(V_{2}\right)=\exp \left[-N \int\left(V_{1}-V_{2}\right) d \mu\right]
$$

Evidently, Jensen's inequality is saturated, implying that the integrand $\sum^{N}\left(V_{1}-V_{2}\right)$ is a constant, $c$, a.e. [ $\left.d \sigma_{V_{2}}\right]$. But, since $V_{2} \in \mathscr{V}, d \sigma_{V_{2}}$ is equivalent to $d \lambda$, which is in turn equivalent to $d^{N} \mu$. Hence the measures $d \sigma_{V_{2}}$ and $d^{N} \mu$ are equivalent. (This is a 
crucial point. Equivalence of the measures fails for the hard core case!) Thus $\sum^{N}\left(V_{1}-V_{2}\right)=c$ a.e. $\left[d^{N} \mu\right] \Rightarrow\left(V_{1}-V_{2}\right)=c / N$ a.e. $[d \mu]$.

Corollary. If $\left(V_{n}\right)$ is a maximizing sequence for $\mathfrak{F}$, normalized so that $Z\left(V_{n}\right)=1$, then

$$
\prod^{N} \exp \left[-V_{n}\left(x_{i}\right) / 2\right] \rightarrow \prod^{N} \exp \left[-V\left(x_{i}\right) / 2\right]
$$

strongly in $L^{2}(d \lambda)$.

Proof. This follows immediately from the corollary to Theorem 2.2 and uniqueness of $V$.

Summary. Thus far, the following has been proved. Under the assumption that $W_{+}$ and $e^{-W}$ are in $L^{1}\left(d^{N} \mu\right)=L^{1}\left(\prod^{N}\left[\varrho\left(x_{i}\right) / N\right] d^{N} x\right)$, (thereby excluding the hard core case):

(i) There is precisely one $V=U+\log (\varrho / N)$ such that $\varrho=\varrho_{V}$.

(ii) This $V$ is also the unique $V$ that maximizes $\mathfrak{F}(V)$.

In the next section, we turn to a discussion of some properties of the map $\varrho \rightarrow U$. In Sects. 5 and 6, we shall return to generalizations of the results of this section.

\section{Continuity of the Inverse Map}

Consider an $N$ particle system with fixed interaction $W$ and a sequence of densities $\left(\varrho_{n}\right)$, each of which is admissible in the sense of Eq. (2.4), i.e.

$$
W_{+} \in L^{1}\left(d^{N} \mu_{n}\right) \text { and } e^{-W} \in L^{1}\left(d^{N} \mu_{n}\right)
$$

where

$$
d \mu_{n}=\left(\varrho_{n} / N\right) d x .
$$

Then by the results of Sect. 2, there exists a sequence of potentials $\left(U_{n}\right)$ such that $\varrho_{n}=\varrho\left(U_{n}\right)$. If the sequence of densities converges, in some sense, to an admissible density $\varrho$, then we shall show that the potentials converge to a potential $U$ such that $\varrho=\varrho(U)$, and hence that the inverse map $U(\varrho)$ is (sequentially) continuous.

First we define the topology in which the densities are taken to converge. For fixed $W$, the set of admissible densities is given by

$$
\begin{aligned}
& \mathscr{D}=\left\{\varrho: \Lambda \rightarrow \mathbb{R}^{+}\left\lfloor\varrho \in L^{1}(d x)\left(\|\varrho\|_{1(d x)}=N\right), \prod^{N} \varrho \in L^{1}\left(e^{-W} d^{N} x\right),\right.\right. \\
& \left.\prod^{N} \varrho \in L^{1}\left(W_{+} d^{N} x\right)\right\} .
\end{aligned}
$$

Since the conditions imposed on admissible densities involve product functions on $\Lambda^{N}$, it is natural to define our topology on the product space

$$
\mathscr{D}^{N}=\left\{\prod^{N} \varrho: \Lambda^{N} \rightarrow \mathbb{R}^{+}\lfloor\varrho \in \mathscr{D}\} .\right.
$$

We shall say that a sequence $\left(\prod^{N} \varrho_{n} \in \mathscr{D}^{N}\right)$ converges to $\prod^{N} \varrho \in \mathscr{D}^{N}$ if 


$$
\prod^{N} \varrho_{n} \rightarrow \prod^{N} \varrho \quad \text { strongly in } L^{1}\left(e^{-W} d^{N} x\right)
$$

and

$$
\prod_{n}^{N} \varrho_{n} \rightarrow \prod^{N} \varrho \quad \text { weakly in } \quad L^{1}\left(d^{N} x\right)
$$

and

$$
\int W_{+} \prod^{N} \varrho_{n} d^{N} x \rightarrow \int W_{+} \prod^{N} \varrho d^{N} x
$$

This type of convergence shall be denoted by $\varrho_{n} \rightarrow \varrho$ in $\mathscr{D}^{N}$. Note that $\varrho_{n} \rightarrow \varrho$ in $\mathscr{D}^{N}$ implies that $\varrho$ is admissible. Observe also that condition (3.6) is clearly equivalent to

$$
\varrho_{n} \rightarrow \varrho \quad \text { weakly in } L^{1}(d x) .
$$

Next we specify an appropriate topology for convergence of the external potentials. Recall that the maximizing potential $V$ for some $\varrho \in \mathscr{D}$ satisfies $Z(V)<\infty$, and hence the function $e^{-U}=(\varrho / N) e^{-V}$ satisfies $\prod^{N} e^{-U} \in L^{1}\left(e^{-W} d^{N} x\right)$. Thus we define the space of potentials

$$
\mathscr{U}=\left\{e^{-U}: \Lambda \rightarrow \mathbb{R}^{+} \mid \prod^{N} e^{-U} \in L^{1}\left(e^{-W} d^{N} x\right)\right\},
$$

with the usual $L^{1}\left(e^{-W} d^{N} x\right)$ norm on $\mathscr{U}^{N}$.

The principal result of this section is that the inverse map is continuous with respect to the topologies defined above; namely, if $\varrho_{n} \rightarrow \varrho$ in $\mathscr{D}^{N}$, then $\prod \exp \left(-U_{n}\right) \rightarrow \prod \exp (-U)$ in $\mathscr{U}^{N}$.

Remark. The measure,

$$
d \sigma=e^{-W} d^{N} x
$$

which appears in the definitions of the spaces $\mathscr{D}$ and $\mathscr{U}$ is not, in general, equivalent to $d^{N} x$ on $\Lambda^{N}$. However, if $\left(\varrho_{n}\right)$ is any sequence of densities in $\mathscr{D}$, then $d \sigma$ and $d^{N} x$ are clearly equivalent on $A^{N} \subset \Lambda^{N}$ where $A=\bigcup_{n} \operatorname{supp}\left(\varrho_{n}\right)$ (see definition below). It is only subsets of $\Lambda^{N}$ of this form which are relevant for convergence of sequences in $\mathscr{D}^{N}$ and $\mathscr{U}^{N}$.

Definition. If $\varrho$ is a nonnegative function on a set $\Lambda$, the support of $\varrho$, denoted by $\operatorname{supp}(\varrho)$, is the set $\{x \in \Lambda \mid \varrho(x)>0\}$.

Notation. Let $\left(\varrho_{n}\right)$ be a sequence of densities in $\mathscr{D}$. Then we may construct corresponding sequences of partition functions $\left(Z_{n}\right)$ and functionals $\left(\mathfrak{F}_{n}\right)$ defined on sets of potentials $\left(\mathscr{V}_{n}\right)$, where $Z_{n}, \mathfrak{F}_{n}$, and $\mathscr{V}_{n}$ are given by Eqs. (2.8), (2.10), and (2.11) with $\varrho$ replaced by $\varrho_{n}$. If $\varrho_{n} \rightarrow \varrho$ in $\mathscr{D}^{N}$, then we construct the analogous quantities $Z, \mathfrak{F}$, and $\mathscr{V}$ for the limit function $\varrho$.

We may also define the quantities $R_{n}=\sup _{S \in \mathscr{V}_{n}} \mathfrak{F}_{n}(S)$, as in Eq. (2.15), and the analogous quantity $R$ for the limit function $\varrho$.

$V=V(\varrho)$ will refer to the maximizing potential for the limit functional $\mathfrak{F}$ and $U=U(\varrho)=-\log (\varrho / N)+V(\varrho)$ will refer to the corresponding external potential. 
The following lemma demonstrates that $R(\varrho)$, which is finite by Proposition 2.1(a), is sequentially lower semicontinuous in $\varrho$.

Lemma 3.1. Suppose $\varrho_{n} \rightarrow \varrho$ in $\mathscr{D}^{N}$. Then

$$
\liminf _{n \rightarrow \infty} R_{n} \geqq R .
$$

Proof. Let $\left(S_{m}\right)$ be a maximizing sequence for $\mathfrak{F}$ in $\mathscr{V}$. It is clear that without loss of generality we may take $S_{m} \in L^{\infty}$. Then, for all $m$ and $n, S_{m} \in \mathscr{V}_{n}$. Hence

$$
R_{n}=\sup _{S \in \mathscr{V}_{n}} \mathfrak{F}_{n}(S) \geqq \mathfrak{F}_{n}\left(S_{m}\right)
$$

By hypothesis, Eqs. (3.5) and (3.6a) are satisfied. Thus $\mathfrak{F}_{n}\left(S_{m}\right) \rightarrow \mathfrak{F}\left(S_{m}\right)$. Hence

$$
\liminf _{n \rightarrow \infty} R_{n} \geqq \lim _{n \rightarrow \infty} \mathfrak{F}_{n}\left(S_{m}\right)=\mathfrak{F}\left(S_{m}\right) .
$$

Equation (3.10) follows by taking the limit $m \rightarrow \infty$.

Given a $\mathscr{D}^{N}$ convergent sequence of densities $\left(\varrho_{n}\right)$, Theorem 2.2 insures that there exist corresponding maximizing potentials $\left(V_{n} \in \mathscr{V}_{n}\right)$ from which we may construct the sequence $\left(\left(\varrho_{n} / N\right) e^{-V_{n}} \in \mathscr{U}\right)$. The next proposition establishes that the latter sequence does indeed converge and that the limit function defines a potential $\hat{V}$. Theorem 3.3 shows that the $\hat{V}$ so defined maximizes $\mathfrak{F}$, and hence that the inverse map is continuous.

Proposition 3.2. Suppose $\left(\varrho_{n}\right)$ converges to $\varrho$ in $\mathscr{D}^{N}$. Let $\left(V_{n} \in \mathscr{V}_{n}\right)$ be the corresponding sequence of maximizing potentials, normalized so that $Z_{n}\left(V_{n}\right)=1$. Let $U_{n}=-\log \left(\varrho_{n} / N\right)+V_{n}$, and construct the sequence $\left(F_{n}\right)$ of functions $F_{n}=\prod \exp \left(-U_{n} / 2\right)$. Then, for some subsequence,

$$
F_{n} \rightarrow F \quad \text { weakly in } L^{2}(d \sigma) .
$$

Moreover, there exists a function $\hat{U}: \Lambda \rightarrow \mathbb{R}$ such that

$$
F=\prod^{N} \exp (-\hat{U} / 2) \text {. }
$$

Proof. $F_{n}$ is norm bounded in $L^{2}(d \sigma)$ [since $Z_{n}\left(V_{n}\right)=1$ ], and hence a convergent subsequence exists by the Banach-Alaoglu theorem. Using Theorem A.3, the form of the weak limit follows from an earlier remark on the equivalence of the measure $d \sigma$ to the underlying product measure $d^{N} x$.

Remark. We may define the potential $\hat{V}$ by setting

$$
e^{-\hat{U}}=(\varrho / N) e^{-\hat{V}} \quad \text { whenever } \quad \varrho(x)>0 .
$$

When $\varrho(x)=0$, the definition of $\hat{V}(x)$ is arbitrary; we set $\hat{V}(x)=0$ when $\varrho(x)=0$. Then

$$
e^{-\hat{U}} \geqq(\varrho / N) e^{-\hat{V}} \text { a.e. }\left[d^{N} x\right] .
$$

Corollary. $\prod^{N} \varrho_{n} \exp \left(-V_{n} / 2\right) \rightarrow \prod^{N} \varrho \exp (-\hat{V} / 2)$ weakly in $L^{1}(d \sigma)$. 
Proof. By hypothesis, $\prod \varrho_{n} \rightarrow \prod \varrho$ strongly in $L^{1}(d \sigma)$, and thus $\prod \varrho_{n}^{1 / 2} \rightarrow \prod \varrho^{1 / 2}$ strongly in $L^{2}(d \sigma)$ (because $|a-b|^{2} \leqq\left|a^{2}-b^{2}\right|$ for $a, b \geqq 0$ ). By the proposition, $F_{n} \rightarrow \prod \exp (-\hat{U} / 2)$ weakly in $L^{2}(d \sigma)$. It is easy to verify that the product of an $L^{2}$ strongly convergent sequence and an $L^{2}$ weakly convergent sequence is an $L^{1}$ weakly convergent sequence, and that the limit is the product of the limits of the two factor sequences. Thus $\prod \varrho_{n} \exp \left(-V_{n} / 2\right) \rightarrow \prod(\varrho N)^{1 / 2} \exp [-\hat{U} / 2]$ $=\prod \varrho \exp [-\hat{V} / 2]$ weakly in $L^{1}(d \sigma)$. The last equality follows from the previous remark.

Remark. Note that although $V_{n} \in \mathscr{V}_{n}$, it is not necessarily the case that $V_{n} \in \mathscr{V}$. Hence $\left(V_{n}\right)$ is not in general a maximizing sequence for $\mathfrak{F}$. Also, it is not yet clear that $\hat{V} \in \mathscr{V}$. In Theorem 3.3, we shall prove that in fact $\hat{V}=V$, the maximizing potential for $\mathfrak{F}$.

Theorem 3.3. Under the assumptions of Proposition 3.2, the function $\hat{V}$ defined by Eq. (3.15) maximizes $\mathfrak{F}$. Furthermore

$$
\prod^{N} \varrho_{n}^{1 / 2} \exp \left(-V_{n} / 2\right) \rightarrow \prod^{N} \varrho^{1 / 2} \exp (-\hat{V} / 2) \text { strongly in } L^{2}(d \sigma) .
$$

Proof. Since $Z_{n}\left(V_{n}\right)=1$ and $Z$ is weakly lower semicontinuous in $F$,

$$
\begin{aligned}
1 & =\lim _{n \rightarrow \infty} Z_{n}\left(V_{n}\right)=\lim _{n \rightarrow \infty}\left\|\prod\left(\varrho_{n} / N\right)^{1 / 2} \exp \left(-V_{n} / 2\right)\right\|_{2(d \sigma)} \\
& \geqq\left\|\prod \exp (-\hat{U} / 2)\right\|_{2(d \sigma)} \geqq\left\|\prod(\varrho / N)^{1 / 2} \exp (-\hat{V} / 2)\right\|_{2(d \sigma)}=Z(\hat{V}) .
\end{aligned}
$$

The second inequality in (3.19) follows from the (pointwise) inequality in Eq. (3.16).

We claim that $\hat{V} \in \mathscr{V}$. According to Eq. (3.19), $Z(\hat{V})$ is finite. Thus it suffices to show that $\hat{V} \in L^{1}(d \mu)$. As in the proof of Theorem 2.2, we decompose $\hat{V}$ into positive and negative pieces, $\hat{V}_{+}$and $\hat{V}_{-}$, with $\hat{V}=\hat{V}_{+}-\hat{V}_{-}$. By reasoning analogous to the proof of Theorem 2.2 [cf. Eqs. (2.19) and (2.20)], $Z(\hat{V})$ $<\infty \Rightarrow \hat{V}_{-} \in L^{1}(d \mu)$.

To establish that $\hat{V}_{+} \in L^{1}(d \mu)$, we shall now show that

$$
\exp \left(-\int \hat{V} \varrho d x\right) \geqq \limsup _{n \rightarrow \infty} \exp \left(-\int V_{n} \varrho_{n} d x\right) .
$$

Define the truncated functions

and

$$
\hat{V}_{+}^{k}(x)= \begin{cases}\hat{V}_{+}(x) & \hat{V}_{+}(x) \leqq k \\ k & \hat{V}_{+}(x)>k\end{cases}
$$

and

$$
\hat{V}_{-}^{m}(x)=\left\{\begin{array}{ll}
\hat{V}_{-}(x) & \hat{V}_{-}(x) \leqq m \\
m & \hat{V}_{-}(x)>m
\end{array},\right.
$$

$$
W^{p}(X)= \begin{cases}W(X) & W(X) \leqq p \\ p & W(X)>p\end{cases}
$$

where $k, m$, and $p$ are positive integers. Then by Jensen's inequality

$$
\begin{aligned}
& \exp \left[-\frac{1}{2} \sum^{N} \int\left[V_{n}-\left(\hat{V}_{+}^{k}-\hat{V}_{-}^{m}\right)\right] d \mu_{n}-\int\left(W-W^{p}\right) d^{N} \mu_{n}\right] \\
& \quad \leqq \int \prod^{N}\left[\frac{1}{N} \varrho_{n} \exp \left(-V_{n} / 2\right) \exp \left(+\hat{V}_{+}^{k} / 2\right) \exp \left(-\hat{V}_{-}^{m} / 2\right)\right] \exp \left(W^{p}\right) d \sigma .
\end{aligned}
$$


Noting that $\prod\left[\exp \left(+\hat{V}_{+}^{k} / 2\right) \exp \left(-\hat{V}_{-}^{m} / 2\right)\right] \exp \left(W^{p}\right) \in L^{\infty}(d \sigma)$ and using the corollary to Proposition 3.2, we have that as $n \rightarrow \infty$, the right-hand side of Eq. (3.24) becomes

$$
\int \prod^{N}\left[\frac{1}{N} \varrho \exp (-\hat{V} / 2) \exp \left(+\hat{V}_{+}^{k} / 2\right) \exp \left(-\hat{V}_{-}^{m} / 2\right)\right] \exp \left(W^{p}\right) d \sigma .
$$

Let us consider the limiting form of the terms on the left-hand side of Eq. (3.24). First, since $\hat{V}_{+}^{k}, \hat{V}_{-}^{m} \in L^{\infty}(d x)$ and $\varrho_{n} \rightarrow \varrho$ weakly in $L^{1}(d x)$,

$$
\lim _{n \rightarrow \infty} \int\left(\hat{V}_{+}^{k}-\hat{V}_{-}^{m}\right) \varrho_{n} d x=\int\left(\hat{V}_{+}^{k}-\hat{V}_{-}^{m}\right) \varrho d x .
$$

By the definition of $W^{p}$, for all $n$,

$$
\int\left(W-W^{p}\right) \prod \varrho_{n} d^{N} x=\int\left(W_{+}-W_{+}^{p}\right) \prod \varrho_{n} d^{N} x,
$$

where $W_{+}$and $W_{+}^{p}$ are the positive parts of $W$ and $W^{p}$. Since $W_{+}^{p} \in L^{\infty}\left(d^{N} x\right)$ and $\prod \varrho_{n} \rightarrow \prod \varrho$ weakly in $L^{1}\left(d^{N} x\right)$,

$$
\lim _{n \rightarrow \infty} \int W_{+}^{p} \prod \varrho_{n} d^{N} x=\int W_{+}^{p} \prod \varrho d^{N} x .
$$

Thus in the limit as $n \rightarrow \infty$, Eq. (3.24) becomes

$$
\begin{aligned}
& {\left[\limsup _{n \rightarrow \infty} \exp \left(-\frac{N}{2} \int V_{n} \varrho_{n} d x\right)\right] \exp \left[+\frac{N}{2} \int\left(\hat{V}_{+}^{k}-\hat{V}_{-}^{m}\right) \varrho d x\right] \exp \left[-\int\left(W-W^{p}\right) d^{N} \mu\right]} \\
& \quad \leqq \int \prod^{N}\left[\exp (-\hat{V} / 2) \exp \left(+\hat{V}_{+}^{k} / 2\right) \exp \left(-\hat{V}_{-}^{m} / 2\right)\right] \exp \left(W^{p}\right) d \lambda
\end{aligned}
$$

where $d \lambda=\prod^{N}(\varrho / N) e^{-W} d^{N} x$ is the finite measure introduced in Eq. (2.5).

Note that for all $k$ and $p$

$$
\begin{aligned}
& \prod\left[\exp (-\hat{V} / 2) \exp \left(+\hat{V}_{+}^{k} / 2\right) \exp \left(-\hat{V}_{-}^{m} / 2\right)\right] \exp \left(W^{p}\right) \\
& \quad \leqq \prod\left[\exp (-\hat{V} / 2) \exp \left(+\hat{V}_{+}^{k} / 2\right)\right] \exp \left(W^{p}\right) \\
& \quad \leqq\left\|\prod \exp \left(+\hat{V}^{k} / 2\right)\right\|_{\infty}\left\|\exp \left(W^{p}\right)\right\|_{\infty} \prod \exp (-\hat{V} / 2) .
\end{aligned}
$$

Since $Z(\hat{V})<\infty$, the function on the right-hand side of $(3.31)$ is in $L^{2}(d \lambda)$, and hence (since $d \lambda$ is a finite measure) in $L^{1}(d \lambda)$. Thus we may use dominated convergence to remove the truncation on $\exp \left(-\hat{V}_{-}^{m} / 2\right)$ on the right-hand side of (3.29). Since $\hat{V}_{-}^{m}$ is an increasing sequence, we use monotone convergence of $\hat{V}_{-}^{m}$ to $\hat{V}_{-}$on the lefthand side of (3.29). Then, in the limit $m \rightarrow \infty$,

$$
\begin{gathered}
{\left[\limsup _{n \rightarrow \infty} \exp \left(-\frac{N}{2} \int V_{n} \varrho_{n} d x\right)\right] \exp \left[+\frac{N}{2} \int\left(\hat{V}_{+}^{k}-\hat{V}_{-}\right) \varrho d x\right]} \\
\cdot \exp \left[-\int\left(W-W^{p}\right) d^{N} \mu\right] \leqq \int \prod^{N}\left[\exp \left[\left(\hat{V}_{+}^{k}-\hat{V}_{+}\right) / 2\right]\right] \exp \left[-\left(W-W^{p}\right)\right] d^{N} \mu \leqq 1 .
\end{gathered}
$$

The second inequality follows from the observation that, for all $k$ and $p, \hat{V}_{+} \geqq \hat{V}_{+}^{k}$ and $W \geqq W^{p}$. Finally, by dominated convergence of $\left(W-W^{p}\right)$ and monotone convergence of $\hat{V}_{+}^{k}$, in the limit as $p \rightarrow \infty$ and $k \rightarrow \infty$, Eq. (3.31) becomes

$$
\limsup _{n \rightarrow \infty} \exp \left(-\int V_{n} \varrho_{n} d x\right) \leqq \exp \left(-\int \hat{V}_{+} \varrho d x\right) \exp \left(+\int \hat{V}_{-} \varrho d x\right) .
$$


Recall that, for all $n, Z_{n}\left(V_{n}\right)=1$, and hence $\mathfrak{F}_{n}\left(V_{n}\right)=\exp \left(-\int V_{n} \varrho_{n} d x\right)$. Then by (3.32) and Lemma 3.1

$$
\begin{aligned}
& \exp \left(-\int \hat{V}_{+} \varrho d x\right) \exp \left(+\int \hat{V}_{-} \varrho d x\right) \geqq \limsup _{n \rightarrow \infty} \mathfrak{F}_{n}\left(V_{n}\right) \\
& \geqq \liminf _{n \rightarrow \infty} \mathfrak{F}_{n}\left(V_{n}\right)=\liminf _{n \rightarrow \infty} R_{n} \geqq R>0 .
\end{aligned}
$$

Since $\hat{V}_{-} \in L^{1}(d \mu)$, this implies that $\hat{V}_{+} \in L^{1}(d \mu)$. Hence $\hat{V} \in \mathscr{V}$.

Finally, we show that $\hat{V}$ maximizes $\mathfrak{F}$. Rewrite (3.32) in the form

$$
\exp \left(-\int \hat{V} \varrho d x\right) \geqq \limsup _{n \rightarrow \infty} \exp \left(-\int V_{n} \varrho_{n} d x\right)
$$

and recall from Eq. (3.19) that

$$
Z(\hat{V}) \leqq \lim _{n \rightarrow \infty} Z_{n}\left(V_{n}\right)
$$

Then, by (3.34), (3.35), and (3.33)

$$
\mathfrak{F}(\hat{V}) \geqq \limsup _{n \rightarrow \infty} \mathfrak{F}_{n}\left(V_{n}\right) \geqq R .
$$

Since $\hat{V} \in \mathscr{V}$, evidently the inequality saturates; i.e. $\hat{V}$ maximizes $\mathfrak{F}$.

The saturation of Eq. (3.36) implies that (3.35) must also be saturated. However, recalling the derivation of (3.35) [cf. Eq. (3.19)], this in turn implies that two other inequalities must be saturated. The first yields the equality

$$
\lim _{n \rightarrow \infty}\left\|\prod\left(\varrho_{n} / N\right)^{1 / 2} \exp \left(-V_{n} / 2\right)\right\|_{2(d \sigma)}=\left\|\prod \exp (-\hat{U} / 2)\right\|_{2(d \sigma)} .
$$

Combining the above norm convergence with the weak convergence in Eq. (3.13), we conclude that the subsequence converges strongly in $L^{2}(d \sigma)$,

$$
\prod\left(\varrho_{n} / N\right)^{1 / 2} \exp \left(-V_{n} / 2\right) \rightarrow \prod \exp (-\hat{U} / 2) .
$$

Saturation of the second inequality in Eq. (3.19) implies that

$$
\left\|\prod \exp (-\hat{U} / 2)\right\|_{2(d \sigma)}=\left\|\prod(\varrho / N)^{1 / 2} \exp (-\hat{V} / 2)\right\|_{2(d \sigma)} .
$$

Then, by the pointwise inequality in Eq. (3.16) and the equivalence of the measures $d \sigma$ and $d^{N} x$, this implies

$$
\prod \exp (-\hat{U} / 2)=\prod(\varrho / N)^{1 / 2} \exp (-\hat{V} / 2) \text { a.e. }[d \sigma]
$$

Hence for the subsequence under consideration

$$
\prod \varrho_{n}^{1 / 2} \exp \left(-V_{n} / 2\right) \rightarrow \prod \varrho^{1 / 2} \exp (-\hat{V} / 2) \text { strongly in } L^{2}(d \sigma) .
$$

Since $\hat{V}$ maximizes $\mathfrak{F}$, by Theorem $2.3, \hat{V}$ satisfies $\varrho=\varrho_{\hat{V}}$ and hence, by Theorem 2.4, $\hat{V}$ is unique up to a constant a.e. $[d \mu]$. Here this constant is fixed by the saturation of Eq. (3.19) which yields $Z(\hat{V})=1$. By uniqueness, the entire sequence converges in the sense of Eq. (3.41), which is the desired result.

Corollary. The inverse map is sequentially continuous in the sense that if $\varrho_{n} \rightarrow \varrho$ in $\mathscr{D}^{N}$, and if $\left(U_{n}\right)$ is the corresponding sequence of external potentials defined by 
$\varrho_{n}=\varrho\left(U_{n}\right)$, then

$$
\prod^{N} \exp \left(-U_{n}\right) \rightarrow \prod^{N} \exp (-U) \text { strongly in } L^{1}(d \sigma),
$$

where $U$ is the potential such that $\varrho=\varrho(U)$.

Moreover, there is a subsequence such that

$$
U_{n}(x) \rightarrow U(x) \text { a.e. }[d x] .
$$

Proof. (3.42) follows immediately from the theorem. Then by strong convergence, there is a subsequence such that $\sum_{i=1}^{N} U_{n}\left(x_{i}\right) \rightarrow \sum_{i=1}^{N} U\left(x_{i}\right)$ a.e. $[d \sigma]$. Thus, (3.43) follows from equivalence of the measures $d \sigma$ and $d^{N} x$.

Remark. Equation (3.42) gives us convergence of $\exp \left(-\sum U_{n}\left(x_{i}\right)\right)$ to $\exp \left(-\sum U\left(x_{i}\right)\right)$ in a strong sense; however, it is convergence on the product space $\left\langle\Lambda^{N}, d \sigma\right\rangle$. Although (3.42) implies (3.43), it does not give us convergence of the entire sequence on the single particle space $\langle\Lambda, d x\rangle$. The reason for this difficulty is that typically $V_{n} \notin \mathscr{V}$ and thus, although $\hat{V}$ maximizes $\mathfrak{F},\left(V_{n}\right)$ is not a maximizing sequence for $\mathfrak{F}$. If $W \in L^{\infty}, \mathscr{D}$ may be replaced by $L^{1}(d x)$ (cf. Proposition 4.1) and it is easy to show that $\left(V_{n}\right)$ is a maximizing sequence. The proof of continuity then follows from the corollary to Theorem 2.4 and we find that $\exp [-U(\varrho)]$ is a continuous map from $L^{1}(d x)$ to $L^{1}(d x)$.

The special features of the case $W \in L^{\infty}$ will be discussed in some detail in the next section on differentiability.

\section{Differentiability of the Inverse Map for Bounded $W$}

In this section we prove the existence of a Fréchet derivative of the inverse map for $W \in L^{\infty}$. In the general case, $W_{+} \in L^{1}\left(d^{N} \mu\right)$, there are intrinsic difficulties with the question of differentiability which we shall not pursue here. We emphasize that although we take $W \in L^{\infty}$, we only require that $\varrho \in L^{1}(d x)$. This of course allows the density to be quite singular.

When $W \in L^{\infty}$, it will be shown below that all positive $L^{1}$ functions of norm $N$ are admissible densities and the maximizing potentials, $V$, are always in $L^{\infty}$. These properties enable us to make use of the Banach space implicit function theorem to show the existence of a Fréchet derivative. Definition. To say that $V(\varrho)$ has a Fréchet derivative at $\varrho$ with kernel $\frac{\delta V}{\delta \varrho}(\varrho ; x, y)$
means that if $\varrho \in L^{1}(d x)$ and $\delta \varrho \in L^{1}(d x)$, then

$$
V(\varrho+\delta \varrho ; x)=V(\varrho ; x)+\int \frac{\delta V}{\delta \varrho}(\varrho ; x, y) \delta \varrho(y) d y+o(\delta \varrho)(x),
$$

where $o(\delta \varrho)$ is a function with the property that $\|o(\delta \varrho)\|_{1(d x)} /\|\delta \varrho\|_{1(d x)} \rightarrow 0$ as $\|\delta \varrho\|_{1(d x)} \rightarrow 0$. More precisely, this latter condition means that there exists a function $\phi(t), t>0$ such that $\phi(t) / t \rightarrow 0$ as $t \rightarrow 0$ and $\|o(\delta \varrho)\|_{1(d x)} \leqq \phi\left(\|\delta \varrho\|_{1(d x)}\right)$.

Remark. Without loss of generality, $e^{-W}$ may always be replaced by $\left(e^{-W}\right)_{\text {sym }}$, the symmetric part of $e^{-W}$, since the definition of $Z(V)$ in Eq. (2.8) remains unchanged 
and the variational derivative of $\mathfrak{F}$ in Eq. (2.13) is unaffected. This amounts to replacing $W$ by $-\log \left[\left(e^{-W}\right)_{\text {sym }}\right]$. The new $W$ is clearly still in $L^{\infty}$. Henceforth we shall drop the subscript sym and assume that $e^{-W}$ has been symmetrized. The definition of $\varrho_{V}$, Eqs. (2.7)-(2.9), becomes

$$
\varrho_{V}=N[Z(V)]^{-1} \int e^{-W} \prod^{N}(\varrho / N) e^{-V} d^{N-1} x .
$$

Proposition 4.1. If $W \in L^{\infty}\left(d^{N} x\right)$, then [see (3.3)]:

(a)

$$
\mathscr{D}=\left\{\varrho: \Lambda \rightarrow \mathbb{R}^{+} \mid \varrho \in L^{1}(d x),\|\varrho\|_{1(d x)}=N\right\} .
$$

(b) For every $\varrho \in \mathscr{D}, V(\varrho) \in L^{\infty}(d \mu)$.

Proof. (a) By definition, if $\varrho \in \mathscr{D}$ as given by (3.3), then $\varrho \in \mathscr{D}$ as given by (4.3).

If $W \in L^{\infty}\left(d^{N} x\right)$ and $\varrho \in \mathscr{D}$ as given by (4.3), then

$$
\int e^{-W} \prod^{N} \varrho d^{N} x \leqq N^{N} \exp \left(\|W\|_{\infty}\right)
$$

and

$$
\int W_{+} \prod^{N} \varrho d^{N} x \leqq N^{N}\left\|W_{+}\right\|_{\infty}
$$

which implies that $\varrho \in \mathscr{D}$ as given by (3.3).

(b) Since $\varrho \in \mathscr{D}$, by Theorems $2.2-2.4$, there exists a unique $V \in \mathscr{V}$ defined a.e. $[d \mu]$ satisfying:

$$
Z(V)=1
$$

and

$$
e^{+V(x)}=\int e^{-W} \prod^{N-1}(\varrho / N) e^{-V} d^{N-1} x .
$$

Condition (i) fixes the normalization of $V$, while condition (ii) implies that $\varrho=\varrho_{V}$. Now by (i)

$$
\left[\exp \left(-\left\|W_{-}\right\|_{\infty}\right)\right]^{1 / N} \leqq \int e^{-V} d \mu \leqq\left[\exp \left(+\left\|W_{+}\right\|_{\infty}\right)\right]^{1 / N},
$$

while by (ii)

$$
\exp \left(-\left\|W_{+}\right\|_{\infty}\right)\left(\int e^{-V} d \mu\right)^{N-1} \leqq e^{+V(x)} \leqq \exp \left(+\left\|W_{-}\right\|_{\infty}\right)\left(\int e^{-V} d \mu\right)^{N-1} .
$$

Equations (4.8) and (4.9) provide a pointwise bound on $V(x)$.

Remark. When $\varrho(x)>0$, the equation satisfied by $V(x)$ is

$$
e^{+V(x)}=\int e^{-W} \prod^{N-1}(\varrho / N) e^{-V} d^{N-1} x .
$$

This equation uniquely extends to the subset of $\Lambda$ on which $\varrho(x)=0$. Since $W \in L^{\infty}\left(d^{N} x\right)$, the $V$ defined by this extension is in $L^{\infty}(d x)$. Therefore, we shall regard (4.10) as defining $V(x)$ for all $x$, not just $\{x \mid \varrho(x)>0\}$.

Lemma 4.2. Suppose $W \in L^{\infty}\left(d^{N} x\right)$. Let $Q: L^{1}(d x) \times L^{\infty}(d x) \rightarrow L^{\infty}(d x)$ be defined by

$$
Q(\varrho, V)=e^{-V} \int e^{-W} \prod^{N-1}(\varrho / N) e^{-V} d^{N-1} x .
$$


Then:

(a) If $\varrho_{0} \in \mathscr{D}$, then there exists a unique $V_{0} \in L^{\infty}(d x)$ such that $Q\left(\varrho_{0}, V_{0}\right)=1$.

(b) $Q(\varrho, V)$ is (norm) continuous in $\varrho$ and $V$.

(c) $\left.\frac{\delta Q}{\delta V}\right|_{\varrho, V}$ exists and is (norm) continuous in $\varrho$ and $V$.

(d) $\left.\frac{\delta Q}{\delta \varrho}\right|_{\varrho, V}$ exists and is (norm) continuous in $\varrho$ and $V$.

(e) $\left[\frac{\delta Q}{\delta V}\right]_{\varrho_{0}, V_{0}}^{-1}$ exists.

Proof. (a) This is an immediate consequence of Proposition 4.1 and the remark following it.

(b), (c), (d) These are straightforward computations. For example,

$$
\begin{aligned}
& \left.\frac{-\delta Q}{\delta V}\right|_{\varrho, V}(h ; y)=h(y) \exp (-V(y)) \int d^{N-1} x e^{-W} \prod_{i=1}^{N-1}\left(\varrho\left(x_{i}\right) / N\right) \exp \left(-V\left(x_{i}\right)\right) \\
& \quad+(N-1) \exp (-V(y)) \int d x h(x)(\varrho(x) / N) \exp (-V(x)) \int d^{N-2} x e^{-W} \\
& \quad \cdot \prod_{i=1}^{N-2}\left(\varrho\left(x_{i}\right) / N\right) \exp \left(-V\left(x_{i}\right)\right)
\end{aligned}
$$

which is jointly $L^{1}$ norm continuous in $\varrho$ and $L^{\infty}$ norm continuous in $V$.

(e) Evaluating (4.12) at $\left(\varrho_{0}, V_{0}\right)$, we obtain

where

$$
\left.\frac{-\delta Q}{\delta V}\right|_{\varrho_{0}, V_{0}}(h ; y)=h(y)+(N-1) \int K(x, y) h(x) d \mu(x),
$$

$K(x, y)=\exp \left(-V_{0}(x)\right) \exp \left(-V_{0}(y)\right) \int d^{N-2} x e^{-W} \prod_{i=1}^{N-2}\left(\varrho_{0}\left(x_{i}\right) / N\right) \exp \left(-V_{0}\left(x_{i}\right)\right)$,

and

$$
d \mu(x)=\left[\varrho_{0}(x) / N\right] d x .
$$

Note that $K(x, y) \in L^{\infty}(d x d y)$.

We claim that the map

$$
T=-\left.\frac{\delta Q}{\delta V}\right|_{\varrho_{0}, V_{0}}: L^{\infty}(d x) \rightarrow L^{\infty}(d x)
$$

has a bounded inverse. However, in order to employ the standard theorems on invertibility of linear operators, it is convenient to view $T$ as an operator on an appropriately chosen Hilbert space. Hence let $h \in L^{2}(d \mu)$. Then

$$
|T h(y)-h(y)| \leqq(N-1)\|K\|_{\infty}\|h\|_{2(d \mu)} .
$$

Since $d \mu$ is a finite measure, Eq. (4.17) implies that $T h \in L^{2}(d \mu)$. Thus $T$ is a linear operator on the Hilbert space $\mathscr{H}=L^{2}(\Lambda, d \mu)$.

We show that $T: L^{2}(d \mu) \rightarrow L^{2}(d \mu)$ is invertible. Since $d \mu$ is finite, $L^{\infty}(d \mu(x) d \mu(y)) \subset L^{2}(d \mu(x) d \mu(y)) \Rightarrow K(x, y) \in L^{2}(d \mu(x) d \mu(y))$. It follows that the operator $A$ defined by

$$
(A h)(y)=-(N-1) \int K(x, y) h(x) d \mu(x)
$$


is Hilbert-Schmidt and hence compact on $\mathscr{H}=L^{2}(\Lambda, d \mu)$. Thus in order to prove that $T=I-A$ is invertible, by the Fredholm alternative, it suffices to show that the equation $A h=h$ possesses only the trivial solution $h=0$. Assume $A h=h$ for some $h \in L^{2}(d \mu)$, i.e.

$$
0=h(y)+(N-1) \int K(x, y) h(x) d \mu(x) .
$$

Multiplying by $\int h(y) d \mu(y)$ and recalling (4.11), (4.14), and $Q\left(\varrho_{0}, V_{0}\right)=1$, a little algebra yields

$$
\begin{aligned}
0 & =\int h^{2}(y) d \mu(y)+(N-1) \int K(x, y) h(x) h(y) d \mu(x) d \mu(y) \\
& =\frac{1}{N} \int e^{-W} \prod^{N} e^{-V_{0}}\left[\sum_{i=1}^{N} h\left(x_{i}\right)\right]^{2} d^{N} \mu .
\end{aligned}
$$

By the equivalence of the measures $e^{-W} \prod^{N} e^{-V_{0}} d^{N} \mu$ and $d^{N} \mu$, Eq. (4.20) implies that $\sum^{N} h\left(x_{i}\right)=0$ a.e. $\left[d^{N} \mu\right]$ and hence $h(x)=0$ a.e. $[d \mu]$. Thus $T: L^{2}(d \mu) \rightarrow L^{2}(d \mu)$ is invertible.

It follows that if $H \in L^{2}(d \mu)$, then there exists a unique $h \in L^{2}(d \mu)$ satisfying $T h=H$, i.e. on the set $\Gamma \equiv \operatorname{supp}\left(\varrho_{0}\right)$,

$$
h(y)=H(y)-(N-1) \int K(x, y) h(x) d \mu(x) .
$$

Now let $H \in L^{\infty}(d x) \subset L^{2}(d \mu)$. By (4.17), it is clear that the unique $h \in L^{2}(d \mu)$ satisfying (4.21) is in $L^{\infty}(d x)$ on $\Gamma$. Moreover, we claim that (4.21) defines a unique $h \in L^{\infty}(d x)$ on all of $\Gamma$. To see this, write $h$ in the form $h(x)=h_{\Gamma}(x)+h_{\Gamma^{c}}(x)$, where $h_{\Gamma}$ is supported on $\Gamma$ and $h_{\Gamma^{c}}$ is supported on $\Gamma^{c}=\Lambda \backslash \Gamma$. By the above reasoning $h_{\Gamma} \in L^{\infty}(d x)$ is unique. The function $h_{\Gamma^{c}} \in L^{\infty}(d x)$ is uniquely determined by the equation

$$
h_{\Gamma^{c}}(y)=\chi_{\Gamma^{c}}\left[H(y)-(N-1) \int K(x, y) h_{\Gamma}(x) d \mu(x)\right] .
$$

Evidently, $H \in L^{\infty}(d x)$ implies that there exists $h \in L^{\infty}(d x)$ such that $T h=H$, i.e. $T: L^{\infty}(d x) \rightarrow L^{\infty}(d x)$ has a bounded inverse.

Corollary. If $W \in L^{\infty}\left(d^{N} x\right)$, then the inverse map $V(\varrho)$ possesses a Fréchet derivative.

Proof. Conditions (a)-(e) of Lemma 4.2 are sufficient, by the implicit function theorem for Banach spaces, to insure that $V(\varrho)$ has a Fréchet derivative. In particular, if $\varrho_{0} \in \mathscr{D}$, the Fréchet derivative of $V(\varrho)$ at $\varrho_{0}$ is given by

$$
\left.\frac{\delta V}{\delta \varrho}\right|_{\varrho_{0}, V\left(\varrho_{0}\right)}=\left[\left.\frac{\delta Q}{\delta V}\right|_{\varrho_{0}, V\left(\varrho_{0}\right)}\right]^{-1}\left[\left.\frac{\delta Q}{\delta \varrho}\right|_{\varrho_{0}, V\left(\varrho_{0}\right)}\right] .
$$

Remarks. (i) According to standard theory, the right side of Eq. (4.23) may be constructed in terms of eigenfunctions of the operator $A$ in Eq. (4.18). Clearly, $A$ is self-adjoint as a map from $L^{2}(d \mu)$ to $L^{2}(d \mu)$. In applying the results of Fredholm theory, it is important to restrict the kernel $K$ to the set $\Gamma \times \Gamma$, where $\Gamma=\operatorname{supp}\left(\varrho_{0}\right)$, as in the proof of Lemma 4.2e. Thus only the function $h_{\Gamma}$ should be expanded in eigenfunctions of $A$. The function $h_{\Gamma^{c}}$ is then uniquely determined by Eq. (4.22). 
(ii) The kernel $\frac{\delta V}{\delta \varrho}(\varrho ; x, y)$ is of independent physical significance. As shown in [5], this kernel is precisely the Ornstein-Zernike direct correlation function. Thus Lemma 4.2 constitutes a proof of the existence of the direct correlation function of a non-uniform fluid.

\section{The Inverse Problem for Several Species}

In this section, we generalize the original inverse problem to include the case of several species of particles. As before, we do not assume that the interaction $W$ has any particular symmetry. Hence the distinction between different species is on the basis of their response to the external field(s).

There are two distinct problems which may be regarded as the inverse problem for several species of particles. The distinction between these two cases is perhaps best understood in terms of an example.

Examples. Consider a system consisting of two nucleons. The single particle space is taken to be the cartesian product $A=X \times\{p, n\}$ where $X \subset \mathbb{R}^{3}$ and $\{p, n\}$ is the nucleon (iso-spin) state space. Then the external potential and the density are functions of the variable $x=(\mathbf{x}, I) \in \Lambda$, i.e. $U(\mathbf{x}, I)=\left(U_{p}(\mathbf{x}), U_{n}(\mathbf{x})\right)$ and $\varrho(\mathbf{x}, I)$ $=\left(\varrho_{p}(\mathbf{x}), \varrho_{n}(\mathbf{x})\right)$. Hence one applies two different potentials and obtains two distinct densities.

The results of Sect. 2 provide conditions under which the application of a given pair of potentials $\left(U_{p}, U_{n}\right)$ produces preprescribed densities $\left(\varrho_{p}, \varrho_{n}\right)$. Notice, however, that as a consequence of condition (1.4), the individual densities include contributions from several terms. For example, $\varrho_{p}(x)$ is an average of the proton densities in three states: the two proton state, the proton-neutron state and the neutron-proton state.

On the other hand, suppose we know that there is exactly one nucleon of each type in the system. Physically, this is not the same as the situation above, because here the proton and neutron are regarded as different species. We may again ask whether it is possible to apply potentials $U_{p}(\mathbf{x})$ and $U_{n}(\mathbf{x})$ to obtain a given pair of densities. However, in this case, the densities $\varrho_{p}(\mathbf{x})$ and $\varrho_{n}(\mathbf{x})$ will only receive a contribution from a state in which one nucleon of each type is always present.

The solution to the inverse problem for the first example is provided by Sect. 2 . The new problem, which we shall now investigate, is the inverse problem for the case of several species, in the sense of the second example above. Specifically, assume that we have a system of $N$ particles interacting via a potential $W$. Suppose that we are able to apply $A \leqq N$ distinct external potentials, $U_{1}, \ldots, U_{A}$, such that each particle couples linearly to one and only one of the $A$ potentials. This effectively partitions the system into $A$ distinct sectors, each consisting of an integral number $n_{a} \leqq N$ of particles (with $\left.\sum_{a=1}^{A} n_{a}=N\right)$, characterized by the property that the $n_{a}$ particles in the $a^{\text {th }}$ sector are acted upon by the potential $U_{a}$ and are unaffected by $U_{b}$ for $b \neq a$. In light of the obvious physical interpretation, we shall refer to particles in the same sector as members of the same species. We note, however, that this division specifically pertains to the action of the particles in response to an external potential and need not reflect any additional symmetry 
in the system. In particular, $W$ need not have any symmetry, so that particles of the "same species" may be distinguishable.

If $\mathbf{U}=\left(U_{a}: \Lambda \rightarrow \mathbb{R} \mid a=1, \ldots, A\right)$ is a collection of external potentials of the form described above, the partition function of the system is

$$
Z_{\mathbf{U}}=\int \exp \left[-W-\sum_{a=1}^{A} \sum_{a_{i}=1}^{n_{a}} U_{a}\left(x_{a_{\mathrm{t}}}\right)\right] d^{N} x .
$$

Provided that $0<Z_{\mathbf{U}}<\infty$, we may construct individual particle densities

$$
\varrho_{a_{i}}\left(\mathbf{U} ; x_{a_{i}}\right)=Z_{\mathbf{U}}^{-1} \int \exp \left[-W-\sum_{a=1}^{A} \sum_{a_{j}=1}^{n_{a}} U_{a}\left(x_{a_{j}}\right)\right] d x_{1} \ldots d \hat{x}_{a_{i}} \ldots d x_{N}
$$

so that the density of the $a^{\text {th }}$ species is

$$
\varrho_{a}(\mathbf{U} ; x)=\sum_{a_{i}=1}^{n_{a}} \varrho_{a_{\imath}}(\mathbf{U} ; x)
$$

Alternatively, assume that we are given a family of functions $\left(\varrho_{a}: \Lambda \rightarrow \mathbb{R}^{+} \mid a=1, \ldots, A\right)$ such that $\varrho_{a} \in L^{1}(d x)$ with $\left\|\varrho_{a}\right\|_{1}=n_{a}$. The natural extension of the inverse problem is to determine whether there exists a family $\mathbf{U}=\left(U_{a}\right)$ such that $\varrho_{a}(\mathbf{U})=\varrho_{a}$ for every $a$.

Obviously, this form of the inverse conjecture may be applied to systems of particles which are subject to physically distinct forces (e.g., strong and weak). However, as the following example demonstrates, this form is also useful for systems which are not normally regarded as consisting of several species.

Example. Consider a magnet of $N<\infty$ Ising spins interacting via a potential $W\left(\sigma_{1}, \ldots, \sigma_{N}\right), \jmath_{i} \in \Lambda=\{-1,1\}$. In accord with conditions (1.4) and (1.5), we assume $\left|W\left(\diamond_{1}, \ldots, \triangleleft_{N}\right)\right|<\infty$ for all $\jmath_{1}, \ldots, \sigma_{N}$.

Let us first describe how the results of Sect. 2 apply to this system. The density $\varrho(\jmath), \triangleleft= \pm 1$, is simply the average number of spins in the plus or minus state. Expressed in terms of the magnetization $M$,

$$
\varrho(\sigma)=(1 / 2)[(M / g) \sigma+N],
$$

where $g$ is the gyromagnetic ratio of the spins. Theorems $2.2-2.4$ indicate that is possible to find a uniform external magnetic field $h$ which produces any given magnetization satisfying $|M / g| \leqq N$.

Now suppose that the system is partitioned into $A \leqq N$ distinct regions, with $n_{a}$ spins in the $a^{\text {th }}$ region. We may regard each region as a separate species. The density of each species is $\varrho_{a}(\delta)=(1 / 2)\left[\left(M_{a} / g_{a}\right)_{\sigma}+n_{a}\right]$, where $g_{a}$ is the gyromagnetic ratio, assumed to be constant throughout the region. The results of this section, as applied to Ising systems, show that one may obtain any given value $\left|M_{a} / g_{a}\right| \leqq n_{a}$ of the magnetization in each region by proper choice of the magnetic field for that region. In particular, by applying a magnetic field which differs from spin to spin, it is possible to produce any preprescribed set of single site magnetizations satisfying $\left|M_{i} / g_{i}\right| \leqq 1$.

The scheme of the proof is analogous to the single species case. First construct the finite induced measures

$$
d \mu_{a}(x)=\left[\varrho_{a}(x) / n_{a}\right] d x,
$$


and

$$
d^{N} \mu=\prod_{a=1}^{A} \prod_{a_{\imath}=1}^{n_{a}} d \mu_{a}\left(x_{a_{\imath}}\right) .
$$

Again, it is convenient to divide out the ideal gas behavior by considering a family of potentials $\mathbf{V}=\left(V_{a}: \Lambda \rightarrow \mathbb{R} \mid a=1, \ldots, A\right)$ defined by $e^{-U_{a}}=\left(\varrho_{a} / n_{a}\right) e^{-V_{a}}$. By analogy to Eqs. (2.7)-(2.9), we express the densities, $\varrho_{\mathbf{V}, a}(x)$, and the partition function, $Z(\mathbf{V})$, as functions of $\mathbf{V}$. The relevant functional for the multispecies case is

$$
\mathfrak{F}_{A}(\mathbf{V})=[Z(\mathbf{V})]^{-1} \exp \left[-\sum_{a=1}^{A} n_{a} \int V_{a} d \mu_{a}\right],
$$

which is defined on the set of families

$$
\mathscr{V}_{A}=\left\{\mathbf{V}=\left(V_{a} \mid a=1, \ldots, A\right) \mid \forall a V_{a} \in L^{1}\left(d \mu_{a}\right), Z(\mathbf{V})<\infty\right\} .
$$

Our assumptions are analogs of those for the single species case; in particular, we take $W_{+} \in L^{1}\left(d^{N} \mu\right)$ and assume $\mathscr{V}_{A} \neq \emptyset$. As remarked previously, the condition $\mathscr{V}_{A} \neq \emptyset$ may be replaced by $e^{-W} \in L^{1}\left(d^{N} \mu\right)$. We also note that the assumptions imply that the measure $d \lambda=e^{-W} d^{N} \mu$ is equivalent to $d^{N} \mu$ and that $\mathbf{V} \in \mathscr{V}_{A} \Rightarrow Z(\mathbf{V})>0$.

Theorem 5.1 is the multispecies version of the results of Sect. 2. We shall omit those details of the proof which strictly parallel the single species case.

Theorem 5.1. Suppose $W_{+} \in L^{1}\left(d^{N} \mu\right)$ and $\mathscr{V}_{A} \neq \emptyset$. Then there exists $\mathbf{V}=\left(V_{a}\right) \in \mathscr{V}_{A}$ such that $\varrho_{\mathbf{V}, a}(x)=\varrho_{a}(x)$ for each $a=1, \ldots$, A. Moreover, each $V_{a}$ is unique up to a constant a.e. $\left[d \mu_{a}\right]$.

Proof. By Jensen's inequality, $\mathfrak{F}_{A}$ is bounded in $\mathscr{V}_{A}$ [cf. Proposition 2.1(a)], and hence we may construct a maximizing sequence $\left(\mathbf{V}_{k} \in \mathscr{V}_{A}\right)$, where $\mathbf{V}_{k}$ denotes the family of $A$ potentials $\left(V_{a}^{k} \mid a=1, \ldots, A\right)$. Exploiting the translation invariance of $\mathfrak{F}_{A}$ [cf. Proposition 2.1(b)], we take $Z\left(\mathbf{V}_{k}\right)=1$ for all $k$. This insures that we may extract a weak limit in $L^{2}(d \lambda)$ from the sequence $\left(F_{k}\right)$ defined by

$$
F_{k}=\prod_{a=1}^{A} \prod_{a_{i}=1}^{n_{a}} \exp \left[-V_{a}^{k}\left(x_{a_{\mathrm{r}}}\right) / 2\right] .
$$

By Theorem A.3 and obvious symmetry considerations, the weak limit is seen to be of the form

$$
F=\prod_{a=1}^{A} \prod_{a_{\imath}=1}^{n_{a}} \exp \left[-V_{a}\left(x_{a_{\imath}}\right) / 2\right] .
$$

We now show that $\mathbf{V} \equiv\left(V_{a} \mid a=1, \ldots, N\right) \in \mathscr{V}_{A}$. Clearly $Z(\mathbf{V}) \leqq 1$, and hence it suffices to establish that each $V_{a} \in L^{1}\left(d \mu_{a}\right)$. We first prove that $\left[V_{a}\right]_{-} \in L^{1}\left(d \mu_{a}\right)$ for all $a$, where $\left[V_{a}\right]_{-}$denotes the negative part of $V_{a}$. Consider the case $Z(\mathbf{V})=0$. Since $Z(\mathbf{V})=\|F\|_{2(d \lambda)}^{2}$, we must have $F \equiv 0$ a.e. $[d \lambda]$. However, if $F \equiv 0$ we may without loss of generality take $\left[V_{a}\right]_{+} \equiv \infty$ and $\left[V_{a}\right]_{-} \equiv 0$ a.e. $\left[d \mu_{a}\right]$ for all $a$, so that clearly $\left[V_{a}\right]_{-} \in L^{1}\left(d \mu_{a}\right)$. Now assume $Z(\mathbf{V})>0$. Then $F \neq 0$ a.e. $[d \lambda]$. Hence, for each $a$, there exists a set $S_{a} \subset \Lambda$ with $\mu_{a}\left(S_{a}\right)>0$ and a constant $C_{a}<\infty$ such that $\left[V_{a}\right]_{+}<C_{a}$ a.e. $\left[d \mu_{a}\right]$ on $S_{a}$. Now let $\tilde{V}_{a}=V_{a}-C_{a}$, and let $T_{a}=\left\{x \in \Lambda \mid\left[\tilde{V}_{a}\right]_{-}(x)>0\right\}$. Then $\mu_{a}\left(T_{a}\right) \geqq \mu_{a}\left(S_{a}\right)>0 . \quad$ Note $\quad$ that $\quad Z(\mathbf{V}) \leqq 1 \Rightarrow Z(\tilde{\mathbf{V}}) \leqq \exp \left[\sum_{a=1}^{A} C_{a}\right]<\infty$, where 
$\tilde{\mathbf{V}}=\left(\tilde{V}_{a} \mid a=1, \ldots, A\right)$. Following the derivation of Eq. (2.20), we find

$$
\left[-\sum_{a=1}^{A} \sum_{a_{t}=1}^{n_{a}} \tilde{V}_{a}\left(x_{a_{\imath}}\right)\right]_{+} \in L^{1}\left(d^{N} \mu\right)
$$

and

$$
\left[-\sum_{a=1}^{A} \sum_{a_{i}=1}^{n_{a}} \tilde{V}_{a}\left(x_{a_{1}}\right)\right]_{+} \geqq\left(\prod_{a=1}^{A} \prod_{a_{l}=1}^{n_{a}} \chi_{T_{a}}\left(x_{a_{\imath}}\right)\right)\left(\sum_{a=1}^{A} \sum_{a_{l}=1}^{n_{a}}\left[\tilde{V}_{a}\right]_{-}\left(x_{a_{i}}\right)\right)
$$

where $\chi_{T_{a}}$ is the characteristic function of the set $T_{a}$. Then, since $\int \chi_{T_{a}} d \mu_{a}$ $=\mu_{a}\left(T_{a}\right)>0$, it is clear that $\left[\tilde{V}_{a}\right]_{-} \in L^{1}\left(d \mu_{a}\right) \Rightarrow\left[V_{a}\right]_{-} \in L^{1}\left(d \mu_{a}\right)$ for all $a$.

Truncating the functions $V_{a}$ and proceeding in a manner entirely analogous to the proof of inequality (2.28), we obtain

$$
\lim _{k \rightarrow \infty} \exp \left(-\sum_{a=1}^{A} n_{a} \int V_{a}^{k} d \mu_{a}\right) \leqq \exp \left(-\sum_{a=1}^{A} n_{a} \int V_{a} d \mu_{a}\right) .
$$

This inequality simultaneously completes the proof that, for all $a$, $V_{a} \in L^{1}\left(d \mu_{a}\right) \Rightarrow \mathbf{V} \in \mathscr{V}_{A}$, and establishes that $\mathbf{V}$ indeed maximizes $\mathfrak{F}_{A}$.

A standard variational argument along the lines of Theorem 2.3 demonstrates that $\varrho_{\mathbf{v}, a}=\varrho_{a}$ for all $a$. Finally, a simple application of Jensen's inequality (cf. Theorem 2.4), shows that each $V_{a}$ is unique up to a constant a.e. $\left[d \mu_{a}\right]$.

Remark. It is obvious that natural extensions of Sects. 3 and 4 hold for this case; the inverse map is sequentially continuous in the sense of Sect. 3 and, if $W \in L^{\infty}$, there exist (partial) functional derivatives $\frac{\delta V_{a}}{\delta \varrho_{b}}$.

\section{Systems with Massive or Charged Coupling}

The systems that we have already considered are those in which either all particles couple to the external field in exactly the same manner (Sects. 2-4) or those in which distinct particles couple independently to different external fields (Sect. 5). In problems of physical interest, it is often the case that different particles couple to the same external field in fashions characteristic of the particular particles. A prototype of this behavior is a system of charged particles in an electric field. Alternatively, consider (different) massive particles under the influence of a gravitational field. Specifically, we shall examine an $N$ particle system with interaction $W\left(x_{1}, \ldots, x_{N}\right)$ such that the potential energy of the particles in the presence of an external potential, $U(x)$, is of the form

$$
W\left(x_{1}, \ldots, x_{N}\right)+\sum_{i=1}^{N} q_{i} U\left(x_{i}\right)
$$

with $q_{i} \in \mathbb{R}$, the "charges" (or "masses" if $q_{i} \equiv m_{i}>0$ for all $i$ ).

For this system, the partition function $Z(U)$ and the individual particle densities $\varrho_{i}(U ; x)$ are given by the analogs of Eqs. (1.2) and (1.1) with the total potential $W+\sum U$ replaced by the potential (6.1). 
As in previous sections, we may construct the particle density $\varrho(U ; x)$ $=\sum_{i=1}^{N} \varrho_{i}(U ; x)$. However, for systems with charged coupling, the particle density may not be the natural function to consider, in the sense that it does not uniquely characterize the system. For example, consider a charge symmetric Hamiltonian describing a neutral system under the influence of an electric potential $U(x)$. It is clear that the particle density remains unchanged if we replace $U(x)$ by $-U(x)$.

Alternatively, let us examine the "charge" density

$$
C_{U}(x)=\sum_{i=1}^{N} q_{i} \varrho_{i}(U ; x) .
$$

[For the above example, we see that $C_{U}(x)$ changes sign under $U \rightarrow-U$.] The principal result of this section is that, under suitable hypotheses, a given function $C(x): \Lambda \rightarrow \mathbb{R}$ satisfying

$$
\int_{\Lambda} C(x) d x=\sum_{i=1}^{N} q_{i},
$$

is the charge density of the system at some external potential $U$. In other words, for a large class of $L^{1}(d x)$ functions, $C(x)$, there exists a unique external potential $U(x)$ such that $C(x)=C_{U}(x)$.

It is an open problem - which we have not solved with our methods - whether a given particle density $\varrho(x)$ can be produced by some $U(x)$ with the charge coupling (6.1). It is not even clear under which conditions this $U$, if it exists, is unique (the previous example of a neutral system shows that uniqueness does not always hold).

Remark. It is important to distinguish the problems posed in this section and in the previous one. In Sect. 5, the particles couple to different potentials, while here the particles all couple to the same external potential. It is only the coefficients of the coupling (which are given a-priori), that differ from one particle to the next. In Sect. 5, the ability to adjust the different potentials was used to obtain distinct particle densities. Here, we may only adjust a single field in an attempt to reproduce the overall charge density.

Remark. In the previous sections, the ideal gas $(W \equiv 0)$ behavior was isolated by considering a variational problem for the potential $V$ defined by $e^{-U}=(\varrho / N) e^{-V}$. Unfortunately, systems with massive or charged coupling are not tractable in terms of a substitution of the form $e^{-U}=|C| e^{-V}$, and hence we attempt to find a maximizing $U$ directly. The difference between the variational problems for $U$ and $V$ manifests itself in two ways. First, we find that we must require slightly stronger integrability conditions for the given density. The second point concerns specification of the support of the given density, which is treated separately for the massive and charged cases. These two points are discussed below.

(i) For the variational problem in terms of $V$, it was found (cf. Theorem 2.2) that the maximizing $V$ always satisfies $V \in L^{1}(\varrho d x)$. Thus for those cases in which the corresponding $U=-\log (\varrho / N)+V$ also happens to satisfy $U \in L^{1}(\varrho d x)$, it follows that $\int \varrho|\log \varrho|<\infty$. Since, in systems with massive or charged coupling, we 
shall attempt to find a maximizing $U$ directly, we shall impose analogs of the condition $\int \varrho|\log \varrho|<\infty$.

(ii) In addition to dividing out the ideal gas behavior, the decomposition $e^{-U}=\varrho e^{-V}$ served to introduce "walls" into the system which cut off the density if the given $\varrho$ was not supported on all of $\Lambda$. For massive particles (i.e., $q_{i} \equiv m_{i}>0$ for all $i$ ), the given density $C(x)$ may still be cut off by setting $e^{-U}=\chi_{\Lambda^{\prime}}, e^{-\tilde{U}}$, where $\chi_{\Lambda^{\prime}}$ is the characteristic function of the set $\Lambda^{\prime}=\{x \in \Lambda \mid C(x)>0\}$. We may then do a variational problem in terms of the potential $\tilde{U}$ on the space $\left\langle\Lambda^{\prime}, d x\right\rangle$ with the constraint that $C>0$. Once we have found the maximizing potential $\tilde{U}: \Lambda^{\prime} \rightarrow \mathbb{R}$, the solution to the original problem is given by

$$
U(x)= \begin{cases}\tilde{U}(x) & x \in \Lambda^{\prime} \\ \infty & x \in \Lambda \backslash \Lambda^{\prime}\end{cases}
$$

For the general case of a charged plasma, there is no simple analog of the above decomposition. In particular, the presence of negative charges would make a substitution of the form (6.4) meaningless.

It is important to note that charge densities may vanish for either of two reasons. It may be the case that no particles are physically present in a given region, or it may be that particles are present, but the region is (pointwise) charge neutral. Thus our inability to cut off the density via a decomposition of the form described above is not a serious difficulty for the solution of the inverse problem, since $C_{U}(x)$ may be made to vanish by arranging charge cancellation. However, if one wishes to produce a charge density in which particles are physically confined to some region of space $\Lambda^{\prime} \subset \Lambda\left[\Rightarrow C(x)=0\right.$ on $\left.\Lambda \backslash \Lambda^{\prime}\right]$, then one must have $e^{-W}=0$ on $\Lambda^{N} \backslash \Lambda^{\prime N}$. This reflects the fact that, while massive particles may be confined to any region of space by the action of a pure gravitational field, no pure electric field can confine a plasma.

In subsequent analysis, we shall restrict attention to $\Lambda^{\prime} \subset \Lambda$ and impose conditions which insure that $e^{-W}>0$ a.e. on $A^{\prime N}$. Then, if the given charge density $C(x)$ vanishes on some subset of $\Lambda^{\prime}$, we shall produce a $U$ such that $C_{U}(x)$ is the canonical density of a system with charge cancellation in that region.

Within this framework, the relevant functional for both the massive and charged cases is

$$
\mathfrak{F}_{C}(U)=\exp \left(-\int C(x) U(x) d x\right) / \int \exp \left[-W-\sum_{i=1}^{N} q_{i} U\left(x_{i}\right)\right] d^{N} x .
$$

Here the integrals in both the numerator and the denominator are over $\Lambda^{\prime}$.

Since $\Lambda^{\prime}$ is the relevant space for the proof of all theorems in this section, we shall take the underlying single particle space to be $\left\langle\Lambda^{\prime}, d x\right\rangle$ (thus in the massive case $C>0$ a.e.). For notational convenience, we henceforth omit the prime.

Let $q_{1}, \ldots, q_{N}$ be the charges of the system. Without loss of generality, we may assume that all charges are nonzero (i.e., integrate out all charge neutral coordinates and redefine $W$ accordingly). Let

$$
A=\left\{i \mid q_{i}>0\right\}, \quad B=\left\{i \mid q_{i}<0\right\}
$$

be the indices of the positive and negative charges, respectively. Define 


$$
Q_{+}=\sum_{a \in A} q_{a}>0 \quad \text { and } \quad Q_{-}=-\sum_{b \in B} q_{b} \geqq 0 .
$$

By convention, we take $Q_{+} \geqq Q_{-}$. Let $C_{+}(x)$ and $C_{-}(x)$ denote the positive and negative parts of $C(x)$, respectively, e.g. $C_{+}(x)=\max (C(x), 0)$. We shall assume that the given charge density satisfies

$$
Q_{-}>\int C_{-} d x \text { unless } Q_{-}=0 .
$$

Clearly, this implies that $Q_{+} \geqq \int C_{+} d x$ with equality only in the massive case. The physical reason for such an assumption is obvious; no electric field can cause (particle) densities of positive and negative charges to have disjoint supports. Thus condition (6.8) is necessary.

Due to charge cancellation, the positive and negative pieces of $C(x)$ do not represent the charge densities of particles of a particular sign; $C_{+}(x)$ and $C_{-}(x)$ are simply excess charge densities. Thus, it is useful to construct functions which simulate the actual densities of positively and negatively charged particles. Such a decomposition is given below.

Definition. Let $Q_{+}$and $Q_{-}$denote the total positive and negative charges of the system. Suppose $C: \Lambda \rightarrow \mathbb{R}$ is an $L^{1}(d x)$ function satisfying $\int C d x=Q_{+}-Q_{-}$. Let $\gamma: \Lambda \rightarrow \mathbb{R}$ be a strictly positive $L^{1}(d x)$ function with $\|\gamma\|_{1(d x)}=1$, and $\Delta \in \mathbb{R}$ a nonnegative constant. Then the functions $\alpha, \beta: \Lambda \rightarrow \mathbb{R}$ defined by

$$
\begin{aligned}
& \alpha(x)=C_{+}(x)+\Delta \gamma(x), \\
& \beta(x)=C_{-}(x)+\Delta \gamma(x),
\end{aligned}
$$

with $\Delta$ chosen such that

$$
\int \alpha d x / Q_{+}=\int \beta d x / Q_{-} \equiv \lambda,
$$

are called the $\gamma$-decomposition of the density $C$.

Remark. (i) We note that $\alpha$ and $\beta$ mimic the properties of actual particle densities. In particular, since $C \in L^{1}(d x), \alpha, \beta \in L^{1}(d x)$, and $C(x)=\alpha(x)-\beta(x)$. If $\Delta>0$, then $\alpha$ and $\beta$ have support a.e. on $A$. Moreover, the ratio of $\int \alpha$ to $\int \beta$ is precisely the ratio one expects for physical densities.

(ii) For the massive case, this formalism is unnecessary. We simply take $\Delta=0$ so that $\alpha(x)=C(x)>0$ and $\beta(x)=0$. The ratio $\int \beta d x / Q_{-}=0 / 0$ should be interpreted as 1 .

If we consider a charged system (and by this we mean $Q_{+}>\int C_{+}$and $Q_{-}>\int C_{-}$), we must have $\Delta>0$. It is easy to verify that if $Q_{+}>Q_{-}$, then the only consistent choice is

$$
Q_{+}-\int C_{+}=Q_{-}-\int C_{-}=\Delta,
$$

which implies that $\lambda=1$. When $Q_{+}=Q_{-}, \Delta$ is not unique and we may take any $\lambda>0$. This flexibility will be crucial for the proof of the charge neutral case.

The conditions that we impose on the system are as follows. We assume that there exists a $\gamma$-decomposition of the density $C \in L^{1}(d x)$ such that

$$
\alpha \log \alpha \in L^{1}(d x), \quad \beta \log \beta \in L^{1}(d x),
$$


and

$$
W_{+} \in L^{1}\left(\prod_{a \in A} \alpha\left(x_{a}\right) \prod_{b \in B} \beta\left(x_{b}\right) d^{N} x\right) .
$$

We also suppose that the corresponding set of potentials

$$
\mathscr{U}_{\gamma}=\left\{U: \Lambda \rightarrow \mathbb{R} \mid U \in L^{1}(\alpha d x) \cap L^{1}(\beta d x), Z(U)<\infty\right\}
$$

is nonvoid. As before, this last assumption may be replaced by

$$
e^{-W} \in L^{1}\left(\prod_{a \in A} \alpha\left(x_{a}\right) \prod_{b \in B} \beta\left(x_{b}\right) d^{N} x\right) .
$$

We also note that for every $U \in \mathscr{U}_{\gamma}, Z(U)>0$ by Jensen's inequality.

Remark. (i) For massive systems, it was mentioned that the only possible $\gamma$-decomposition is the trivial one: $\alpha(x)=C(x)>0$ and $\beta(x)=0$. In this case, the presence of $b, B$, and $\beta$ in all subsequent expressions is superfluous; they should be replaced by $\emptyset, 0$ or 1 as circumstances dictate. In particular, the requirements on the massive system reduce to $C \in L^{1}(d x)$,

$$
\begin{gathered}
C \log C \in L^{1}(d x), W_{+} \in L^{1}\left(\prod_{i=1}^{N} C\left(x_{i}\right) d^{N} x\right) \text { and } \mathscr{U}_{C}=\left\{U: \Lambda \rightarrow \mathbb{R} \mid U \in L^{1}(C d x),\right. \\
Z(U)<\infty\} \neq \emptyset .
\end{gathered}
$$

(ii) For the charged case, if $C \in L^{1}(d x), \quad|C| \log |C| \in L^{1}(d x)$ and $W_{+} \in L^{1}\left(\prod_{i=1}^{N}\left|C\left(x_{i}\right)\right| d^{N} x\right)<\infty$ a.e., then there always exists a $\gamma$-decomposition of $C$ which satisfies (6.12)-(6.14). Hence the above are sufficient, but not necessary, conditions.

For $U \in \mathscr{U}_{\gamma}$, the numerator of the functional $\mathfrak{F}_{C}(U)$ in Eq. (6.5) may be written as $\exp \left[-\int[\alpha(x)-\beta(x)] U(x) d x\right]$.

It is convenient to define the unit measures

and

$$
\begin{aligned}
& d \mu_{\alpha}(x)=\alpha(x) d x / \lambda Q_{+}, \\
& d \mu_{\beta}(x)=\beta(x) d x / \lambda Q_{-},
\end{aligned}
$$

$$
d^{N} \mu_{\gamma}=\prod_{a \in A} d \mu_{\alpha}\left(x_{a}\right) \prod_{b \in B} d \mu_{\beta}\left(x_{b}\right) .
$$

Remark. It will become clear that the above measures perform some of the roles played by $d \mu$ and $d^{N} \mu$ in previous sections. Except in the massive case, the system is not equipped with a natural positive measure. Furthermore, with our methods, it is not useful to employ $C d x$ as a signed measure. Instead, the (non-unique) $\gamma$-decomposition of $C$ enables us to regard the indefinite sign of $C$ as a manifestation of the nature of the coupling. For example, the integral appearing in the numerator of $\mathfrak{F}_{C}(U)$ may be rewritten as

$$
\begin{aligned}
\int C(x) U(x) d x & =\lambda Q_{+} \int U(x) d \mu_{\alpha}(x)-\lambda Q_{-} \int U(x) d \mu_{\beta}(x) \\
& =\lambda \sum_{i \in A} q_{i} \int U(x) d \mu_{\alpha}(x)+\lambda \sum_{i \in B} q_{i} \int U(x) d \mu_{\beta}(x) \\
& =\lambda \sum_{i=1}^{N} q_{i} \int U\left(x_{i}\right) d^{N} \mu_{\gamma} .
\end{aligned}
$$


Remark. By construction, the measures $d \mu_{\alpha}(x)$ and $d \mu_{\beta}(x)$ are equivalent to $d x$ on $\Lambda$. Thus the conditions $W_{+} \in L^{1}\left(d^{N} \mu_{\gamma}\right)$ and $\mathscr{U}_{\gamma} \neq \emptyset$ imply that $0<e^{-W}<\infty$ a.e. on $\Lambda^{N}$, and hence the measure

$$
d \sigma=e^{-W} d^{N} x
$$

is equivalent to $d^{N} x$ on $\Lambda^{N}$.

The scheme of the existence proof is similar to that of Sects. 2 and 5. We first establish the boundedness of $\mathfrak{F}_{C}$.

Proposition 6.1. If there exists a $\gamma$-decomposition of $C \in L^{1}(d x)$ such that $\alpha \log \alpha \in L^{1}(d x), \beta \log \beta \in L^{1}(d x), W_{+} \in L^{1}\left(d^{N} \mu_{\gamma}\right)$ and $\mathscr{U}_{\gamma} \neq \emptyset$, and if either $Q_{-}=0$ or $Q_{-}>\int C_{-} d x$, then $\mathfrak{F}_{C}(\cdot)$ is (uniformly) bounded above in $\mathscr{U}_{\gamma}$.

Proof. If $U \in \mathscr{U}_{\gamma}$, then the numerator of $\mathfrak{F}_{C}(U)$ may be expressed as in Eq. (6.18). We may also express $Z(U)$ in terms of the measure $d^{N} \mu_{\gamma}$ :

$$
Z(U)=\lambda^{N} Q_{+}^{n_{+}} Q_{-}^{n_{-}} \int \exp \left[-\tilde{W}-\sum_{i=1}^{N} q_{i} U\left(x_{i}\right)\right] d^{N} \mu_{\gamma},
$$

where

$$
\tilde{W}\left(x_{1}, \ldots, x_{N}\right)=W\left(x_{1}, \ldots, x_{N}\right)+\sum_{a \in A} \log \alpha\left(x_{a}\right)+\sum_{b \in B} \log \beta\left(x_{b}\right),
$$

and $n_{+}$and $n_{-}$denote the total number of positive and negative charges, respectively. It follows from the hypotheses that $\tilde{W} \in L^{1}\left(d^{N} \mu_{y}\right)$.

By Jensen's inequality

$$
\mathfrak{F}_{C}(U) \leqq\left[\exp \left(\lambda \int \tilde{W} d^{N} \mu_{\gamma}\right) / \lambda^{N} Q_{+}^{n_{+}} Q_{--}^{n_{-}}\right][Z(U)]^{\lambda-1} .
$$

If $Q_{+}>Q_{-}$, then $\lambda=1$. If $Q_{+}=Q_{-}$, we may take $\lambda=1$ (for the purposes of this proof). In both cases, we see that the above bound is independent of $U$.

Remark. As in previous sections, we shall find the maximizing potential via the limit of sequences of functions of the form $F_{n}=\prod_{i=1}^{N} \exp \left[-q_{i} U_{n}\left(x_{i}\right) / 2\right]$. Even if we could show that these converged weakly, and hence by Theorem A.3 that the weak limit is a product function, there would be no reason to assume that this limit is a product of the same factors raised to various powers. Indeed, it is easy to construct counterexamples to such a conjecture in general. However, Theorem A.4 provides conditions under which the limit function is of the desired form. In the next theorem, we exploit specific properties of our maximizing sequence to demonstrate that there is a subsequence satisfying the conditions of Theorem A.4.

Theorem 6.2. Under the assumptions of Proposition 6.1, there exists a maximizing sequence $\left(U_{n} \in \mathscr{U}_{\gamma}\right)$ such that the sequence of functions $\left(F_{n}\right)$ defined by

$$
F_{n}=\prod_{i=1}^{N} \exp \left[-q_{i} U_{n}\left(x_{i}\right) / 2\right]
$$

has a subsequence which converges strongly in $L^{2}(d \sigma)$

$$
F_{n} \rightarrow F \text {. }
$$


Furthermore, the limit function is of the form

$$
F=\prod_{i=1}^{N} \exp \left[-q_{i} U\left(x_{i}\right) / 2\right]
$$

Proof. By Proposition 6.1, $\mathfrak{F}_{C}(\cdot)$ is bounded above in $\mathscr{U}_{\gamma}$ and thus there exists a maximizing sequence $\left(U_{n} \in \mathscr{U}_{\gamma}\right)$ so that

$$
R=\lim _{n \rightarrow \infty} \mathfrak{F}_{C}\left(U_{n}\right)=\sup _{U \in \mathscr{U}_{\gamma}} \mathfrak{F}_{C}(U)<\infty .
$$

Since $\mathscr{U}_{\gamma} \neq \emptyset, R>0$ and without loss of generality, we may take

$$
\mathfrak{F}_{C}\left(U_{n}\right)>R / 2
$$

for all $n$. If $Q_{+}>Q_{-}$, then by adding constants, the $U_{n}$ may be chosen so that $Z\left(U_{n}\right)=1$. This obviously cannot be accomplished in the charge neutral case since here $Z(U)$ is invariant if $U$ is shifted a constant. However, by Eq. (6.22), we see that for all $\lambda>0$ and all $U \in \mathscr{U}_{\gamma}$

$$
\mathfrak{F}_{C}(U) \leqq \ell(\lambda)[Z(U)]^{\lambda-1},
$$

where $\ell(\lambda)$ is independent of $U$. Thus, by Eq. (6.27), all $U_{n}$ in the maximizing sequence satisfy

$$
R / 2<\ell(\lambda)\left[Z\left(U_{n}\right)\right]^{\lambda-1}
$$

Then by choosing $\lambda>1$ and $\lambda<1$ (as we may do if $Q_{+}=Q_{-}$), it is seen that $Z\left(U_{n}\right)$ is uniformly bounded away from zero and infinity. Let us extract a subsequence of $\left(U_{n}\right)$ [also denoted by $\left.\left(U_{n}\right)\right]$ such that $Z\left(U_{n}\right)$ converges and define

$$
Z_{0}=\lim _{n \rightarrow \infty} Z\left(U_{n}\right)
$$

In the nonneutral case $Z_{0}=1$; however, in general, $0<Z_{0}<\infty$.

Consider the sequence $\left(F_{n}\right)$ defined by

$$
F_{n}=\prod_{i=1}^{N} \exp \left[-q_{i} U\left(x_{i}\right) / 2\right] .
$$

Since $Z\left(U_{n}\right)=\left\|F_{n}\right\|_{2(d \sigma)}^{2}$, the $F_{n}$ are norm bounded in $L^{2}(d \sigma)$, and hence we may extract a further subsequence of $\left(U_{n}\right)$ [again denoted by $\left(U_{n}\right)$ ] such that the corresponding $F_{n}$ converge weakly in $L^{2}(d \sigma)$

$$
F_{n} \rightarrow F \text {. }
$$

By weak convergence, we have

$$
Z_{0}=\lim _{n \rightarrow \infty}\left\|F_{n}\right\|_{2(d \sigma)}^{2} \geqq\|F\|_{2(d \sigma)}^{2} .
$$

We now show that the $F_{n}$ actually converge strongly to $F$. Since weak convergence has already been established, it suffices to show norm convergence, i.e. that $Z_{0}=\|F\|_{2(d \sigma)}^{2}$. Let $\varepsilon>0$. For $n_{0}$ sufficiently large,

$$
n>n_{0} \Rightarrow \mathfrak{F}_{C}\left(U_{n}\right)>R(1-\varepsilon),
$$


and

$$
Z\left(U_{n}\right)>Z_{0}(1-\varepsilon)
$$

Clearly, the subsequence $\left(F_{n} \mid n>n_{0}\right)$ converges weakly to $F$. Thus by Mazur's theorem [9], there exist coefficiently $c_{k}^{(n)} \geqq 0$, with $c_{k}^{(n)}=0$ for $k>n$ and $\sum_{k>n_{0}}^{n} c_{k}^{(n)}=1$, such that the sequence $\left(G_{n} \mid n>n_{0}\right)$ of convex combinations, defined by

$$
G_{n}=\sum_{k>n_{0}}^{n} c_{k}^{(n)} F_{k}
$$

converges strongly in $L^{2}(d \sigma)$

$$
G_{n} \rightarrow F
$$

Hence $\lim _{n \rightarrow \infty}\left\|G_{n}\right\|_{2(d \sigma)}^{2}=\|F\|_{2(d \sigma)}^{2}$. Combining this with Eq. (6.33), we obtain

$$
Z_{0}=\lim _{n \rightarrow \infty}\left\|F_{n}\right\|_{2(d \sigma)}^{2} \geqq\|F\|_{2(d \sigma)}^{2}=\lim _{n \rightarrow \infty}\left\|G_{n}\right\|_{2(d \sigma)}^{2} .
$$

Note that $\left\|G_{n}\right\|_{2(d \sigma)}^{2}$ is given by

$$
\left\|G_{n}\right\|_{2(d \sigma)}^{2}=\sum_{j, k>n_{0}}^{n} c_{j}^{(n)} c_{k}^{(n)} \int F_{j} F_{k} d \sigma
$$

Let $n>n_{0}$. For this $n$, choose $j_{n}$ and $k_{n}$ such that (i) $c_{j_{n}}^{(n)} \neq 0$ and $c_{k_{n}}^{(n)} \neq 0$, and (ii) for all $m$ and $\ell$ with $c_{m}^{(n)} \neq 0$ and $c_{\ell}^{(n)} \neq 0$,

$$
\int F_{m} F_{\ell} d \sigma \geqq \int F_{j_{n}} F_{k_{n}} d \sigma .
$$

Hence $j_{n}$ and $k_{n}$ are chosen to correspond to the minimum integral (with nonzero coefficient) in the sum on the right-hand side of (6.39). Now let us define the function $Y_{n}: \Lambda^{N} \rightarrow \mathbb{R}$ by

$$
Y_{n}^{2}=F_{j_{n}} F_{k_{n}}
$$

and the function $y_{n}: \Lambda \rightarrow \mathbb{R}$ by

$$
y_{n}=\frac{1}{2}\left(U_{j_{n}}+U_{k_{n}}\right)
$$

By construction

$$
\left\|G_{n}\right\|_{2(d \sigma)}^{2} \geqq\left\|Y_{n}\right\|_{2(d \sigma)}^{2}=Z\left(y_{n}\right) .
$$

Clearly $Z\left(y_{n}\right)<\infty$. Moreover, $U_{j_{n}}, \quad U_{k_{n}} \in L^{1}\left(d \mu_{\alpha}\right) \cap L^{1}\left(d \mu_{\beta}\right), \quad$ and hence $y_{n} \in L^{1}\left(d \mu_{\alpha}\right) \cap L^{1}\left(d \mu_{\beta}\right)$. Therefore $y_{n} \in \mathscr{U}_{\gamma}^{\prime}$; in particular, $Z\left(y_{n}\right)>0$ by the remark following (6.15). We have

$$
\begin{aligned}
R= & \sup _{U \in \mathscr{U}_{\gamma}} \mathfrak{F}_{C}(U) \geqq \mathfrak{F}_{C}\left(y_{n}\right) \\
= & \exp \left[-\frac{1}{2} \int C\left(U_{j_{n}}+U_{k_{n}}\right) d x\right] / Z\left(y_{n}\right) \\
= & \left(\mathfrak{F}_{C}\left(U_{j_{n}}\right) Z\left(U_{j_{n}}\right)\right)^{1 / 2}\left(\mathfrak{F}_{C}\left(U_{k_{n}}\right) Z\left(U_{k_{n}}\right)\right)^{1 / 2} / Z\left(y_{n}\right) \\
& >R Z_{0}(1-\varepsilon)^{2} / Z\left(y_{n}\right) .
\end{aligned}
$$


The final inequality follows from the bounds in Eqs. (6.34) and (6.35). Combining (6.43) and (6.44), we obtain, for $n>n_{0}$,

$$
\left\|G_{n}\right\|_{2(d \sigma)}^{2} \geqq Z\left(y_{n}\right)>Z_{0}(1-\varepsilon)^{2} .
$$

Taking the limit as $n \rightarrow \infty$ and comparing this with Eq. (6.38), we see that

$$
Z_{0} \geqq\|F\|_{2(d \sigma)}^{2}>Z_{0}(1-\varepsilon)^{2}
$$

for all $\varepsilon>0$. This establishes strong convergence of the sequence $\left(F_{n}\right)$.

Next we shall show that the limit function $F$ is of the desired form. Since the subsequence $\left(F_{n}\right)$ converges strongly, there is a further subsequence which converges pointwise a.e. on $\Lambda^{N}$. Then, by Theorem A.4, it suffices to show that there is some set $T \subset \Lambda$ of positive measure such that $F>0$ a.e. on $T^{N}$. In fact, we shall show that $F>0$ a.e. on the full space $\Lambda^{N}$.

Suppose $F=0$ on some set $S \subset \Lambda$ with $\sigma(S)>0$. By equivalence of the measures $d^{N} \mu_{\gamma}$ and $d \sigma$, it follows that $\mu_{\gamma}(S)>0$. Let $S^{c}=\Lambda \backslash S$.

Let us write the numerator of $\mathfrak{F}_{C}(U)$ in the form

$$
\begin{aligned}
I(U) & =\exp \left[-\lambda \sum_{i=1}^{N} q_{i} \int_{S} U_{n}\left(x_{i}\right) d^{N} \mu_{\gamma}\right] \exp \left[-\lambda \sum_{i=1}^{N} q_{i} \int_{S^{c}} U_{n}\left(x_{i}\right) d^{N} \mu_{\gamma}\right] \\
& \equiv I_{S}(U) I_{S^{c}}(U) .
\end{aligned}
$$

Since $F_{n} \rightarrow 0$ strongly in $L^{2}(d \sigma)$ on $S$ and since $\mu_{\gamma}(S)>0$, it is clear that

$$
I_{S}\left(U_{n}\right) \rightarrow 0
$$

By Eq. (6.27), for all $n$

$$
0<R / 2<I\left(U_{n}\right) / Z\left(U_{n}\right) .
$$

Since $Z\left(U_{n}\right)$ is uniformly bounded above, it follows that $I\left(U_{n}\right)$ is uniformly bounded below. Hence it must be the case that

$$
I_{S^{c}}\left(U_{n}\right) \rightarrow \infty \text {. }
$$

But, by Jensen's inequality

$$
\left[I_{S^{c}}\left(U_{n}\right)\right]^{\left[1 / \mu_{\gamma}\left(S^{c}\right)\right]} \leqq \xi\left[Z\left(U_{n}\right)\right]^{\lambda}
$$

where $\xi<\infty$ is independent of $U_{n}$, and where $Z\left(U_{n}\right)$ has been obtained by integrating over the larger set $S \cup S^{c}=\Lambda^{N}$. This implies $Z\left(U_{n}\right) \rightarrow \infty$, a contradiction. Hence $F>0$ a.e. on $\Lambda^{N}$.

Remark. It should be pointed out that the equivalence of the measures $d^{N} \mu_{\gamma}$ and $d^{N} x$ is only necessary to show that $F>0$ a.e. on $\Lambda^{N}$. In fact, if we relax the condition that the function $\gamma$ be supported on all of $A$, and merely require that $\gamma>0$ on some set $\Gamma \subset A$ of positive $[d x]$ measure, then we find that $d^{N} \mu_{\gamma}$ is supported on $\Gamma^{N}$. This guarantees that $F>0$ a.e. on $\Gamma^{N}$, which is all that is needed to apply Theorem A.4 and hence prove Theorem 6.2. Thus, one may obtain slightly more flexibility in the choice of the $\gamma$-decomposition of $C$. However, this does not provide any real generalization of the result.

Theorem 6.3. Under the hypotheses of Proposition 6.1, there exists $U$ which maximizes $\mathfrak{\mho}_{C}(\cdot)$ in $\mathscr{U}_{\gamma}$. 
Proof. Let $U: \Lambda \rightarrow \mathbb{R}$ be the function defined by the strong limit in Eq. (6.25). We shall show that $U \in \mathscr{U}_{\gamma}$ and that $\mathfrak{F}_{C}(U)=\sup _{Y \in \mathscr{U}_{\gamma}} \mathfrak{F}_{C}(Y)$. This follows from reasoning which is almost identical to that used in the proofs of Theorems 2.2 and 5.1.

First, we use that fact that $0<Z(U)<\infty$ to show that $U_{-} \in L^{1}\left(d \mu_{\alpha}\right)$ and $U_{+} \in L^{1}\left(d \mu_{\beta}\right)$. This is established by a derivation along the lines of Eq. (5.11), in which one again may have to use regulating constants to guarantee that the relevant sets are of nonzero measure.

Next, we truncate from above each of the functions $q_{i} U$ and the function $\tilde{W}$, defined in Eq. (6.21). Following the derivation of Eq. (2.28), we obtain a numerator inequality of the form

$$
R Z_{0}=\lim _{n \rightarrow \infty} \exp \left[-\int \sum_{i=1}^{N} q_{i} U_{n}\left(x_{i}\right) d^{N} \mu_{\gamma}\right] \leqq \exp \left[-\int \sum_{i=1}^{N} q_{i} U\left(x_{i}\right) d^{N} \mu_{\gamma}\right] .
$$

Since $R Z_{0}>0$, and since $U_{-} \in L^{1}\left(d \mu_{\alpha}\right)$ and $U_{+} \in L^{1}\left(d \mu_{\beta}\right)$, the above inequality establishes that $U \in L^{1}\left(d \mu_{\alpha}\right) \cap L^{1}\left(d \mu_{\beta}\right)$. Thus $U \in \mathscr{U}_{\gamma}$.

Moreover, since $\lim _{n \rightarrow \infty} Z(U)=Z_{0}$, Eq. (6.52) implies that

$$
\mathfrak{F}_{C}(U)=\lim _{n \rightarrow \infty} \mathfrak{F}_{C}\left(U_{n}\right)=\sup _{Y \in \mathscr{U}_{\gamma}} \mathfrak{F}_{C}(Y),
$$

and hence that $U$ maximizes $\mathfrak{\mho}_{C}(\cdot)$ in $\mathscr{U}_{\gamma}$.

Remark. Let $\mathscr{U}_{C}$ be a set of potentials defined by

$$
\mathscr{U}_{C}=\left\{U: \Lambda \rightarrow \mathbb{R} \mid U \in L^{1}(|C| d x), Z(U)<\infty\right\} .
$$

Since $\alpha(x) \geqq C_{+}(x)$ and $\beta(x) \geqq C_{-}(x)$, it is clear that for every $\gamma$-decomposition of $C$, $\mathscr{U}_{\gamma} \subset \mathscr{U}_{C}$, and hence $\sup _{U \in \mathscr{U}_{\gamma}} \mathfrak{F}_{C}(U) \leqq \sup _{U \in \mathscr{U}_{C}} \mathfrak{F}_{C}(U)$. In fact, this inequality is saturated for any $\gamma$-decomposition. This is seen to follow, for example, from the fact that a maximizing sequence for $\mathfrak{F}_{C}(\cdot)$ in either class may be taken in $L^{\infty}$. In particular, this shows that Theorem 6.3 established the existence of a maximum of $\mathfrak{F}_{C}(\cdot)$ in $\mathscr{U}_{C}$.

Next we demonstrate uniqueness of the potential. In contrast to Sect. 2, we shall use the Schwarz, rather than the Jensen inequality.

Proposition 6.4. If there exists $U \in \mathscr{U}_{C}$ which maximizes $\mathfrak{F}_{C}(\cdot)$, then up to a constant $U$ is unique a.e. $[d x]$.

Proof. Suppose there exists $U_{1}, U_{2} \in \mathscr{U}_{C}$ such that $\mathfrak{\mho}_{C}\left(U_{1}\right)=\mathfrak{\mho}_{C}\left(U_{2}\right)=\sup _{U \in \mathscr{U}_{C}} \mathfrak{\mho}_{C}(U)$. Then $(1 / 2)\left(U_{1}+U_{2}\right) \in \mathscr{U}_{C}, \quad$ since clearly $(1 / 2)\left(U_{1}+U_{2}\right) \in L^{1}(|C| d x)$, and $Z\left[(1 / 2)\left(U_{1}+U_{2}\right)\right] \leqq \sqrt{Z\left(U_{1}\right)} \sqrt{Z\left(U_{2}\right)}$ by Schwarz's inequality. Then

$$
\begin{aligned}
\sup _{U \in \mathscr{U}_{C}} \mathfrak{F}_{C}(U) & \geqq \mathfrak{F}_{C}\left[(1 / 2)\left(U_{1}+U_{2}\right)\right] \\
& =\exp \left(-\frac{1}{2} \int C U_{1} d x\right) \exp \left(-\frac{1}{2} \int C U_{2} d x\right) / Z\left[(1 / 2)\left(U_{1}+U_{2}\right)\right] \\
& \geqq\left[\exp \left(-\int C U_{1} d x\right) / Z\left(U_{1}\right)\right]^{1 / 2}\left[\exp \left(-\int C U_{2} d x\right) / Z\left(U_{2}\right)\right]^{1 / 2} \\
& =\sup _{U \in \mathscr{U}_{C}} \mathfrak{F}_{C}(U) .
\end{aligned}
$$


Evidently Schwarz's inequality is saturated. Thus

$$
\prod_{i=1}^{N} \exp \left[-q_{i} U_{1}\left(x_{i}\right)\right]=(\mathrm{const}) \prod_{i=1}^{N} \exp \left[-q_{i} U_{2}\left(x_{i}\right)\right] \text { a.e. }[d \sigma] \text {. }
$$

By the equivalence of the measures $d \sigma$ and $d^{N} x$,

$$
U_{1}(x)=U_{2}(x)+\text { const a.e. }[d x] .
$$

Theorem 6.5. Under the hypotheses of Proposition 6.1, up to a constant there is a unique $U \in \mathscr{U}_{C}$ such that $C_{U}=C$.

Proof. By the standard variational arguments (cf. Theorem 2.3), any $U \in \mathscr{U}_{C}$ which maximizes $\mathfrak{F}_{C}$ satisfies $C_{U}=C$ a.e. By Theorem 6.3 and the subsequent remark, such a $U$ exists and, by Proposition 6.4, it is unique up to a constant. This demonstrates that $\mathfrak{F}_{C}(\cdot)$ has a unique global maximum.

In order to show that $\mathfrak{F}_{C}(\cdot)$ has no other extrema, and hence that there are no other $U \in \mathscr{U}_{C}$ satisfying $C_{U}=C$, it suffices to demonstrate that $\mathfrak{F}_{C}(\cdot)$ is strictly $\log$ concave. We first observe that the numerator, $\exp \left(-\int C U d x\right)$, is log linear. Next let $U_{1}, U_{2} \in \mathscr{U}_{C}$ and $\lambda \in(0,1)$. Then by Hölder's inequality

$$
\log Z\left[\lambda U_{1}+(1-\lambda) U_{2}\right] \leqq \lambda \log Z\left(U_{1}\right)+(1-\lambda) \log Z\left(U_{2}\right) .
$$

The inequality is strict unless $U_{1}=U_{2}+$ const, demonstrating that $Z$ is strictly $\log$ convex and thus that $\mathfrak{F}_{C}(\cdot)$ is strictly log concave.

Remark. An exact analog of Theorem 2.4a applies here, so that no measurable function $U$ in the complement of $\mathscr{U}_{C}$ satisfies $C_{U}=C$.

\section{Summary of Results for the Canonical Ensemble}

In Sects. 2 through 6, we have treated various aspects of the inverse problem for the canonical ensemble. In Sect. 8, we shall examine the analogous problems for the grand canonical ensemble. Here we provide a summary of the results of Sects. 2 through 6 , so that the corresponding theorems for the two ensembles may be easily contrasted.

All results quoted in this section refer to systems of $N<\infty$ classical particles, with coordinates in some $(\sigma$-finite) measure space $\langle\Lambda, d x\rangle$, which interact via a potential $W\left(x_{1}, \ldots, x_{N}\right)$.

In Sect. 2, it was shown that if $W_{+}$and $e^{-W}$ are in $L^{1}\left[\prod_{i=1}^{N} \varrho\left(x_{i}\right) d x_{i}\right]$, then, up to a constant, there is precisely one $V: \Lambda \rightarrow \mathbb{R}$ in the class of all [@dx]-measurable functions such that $\varrho(x)=\varrho_{V}(x)$ (Theorems 2.3 and 2.4). This $V$ satisfies $V \in L^{1}(\varrho d x)$ and, up to a constant, is the unique $V$ which maximizes the functional $\mathfrak{F}(V)=\exp \left(-\int V \varrho d x\right) / Z(V)$ (Theorem 2.2). The function $U=-\log (\varrho / N)+V$ is the external potential which produces the density $\varrho$, i.e. $\varrho(x)=\varrho(U ; x)$.

The continuity of the inverse map $U(\varrho)$ was investigated in Sect. 3. There it was shown (corollary to Theorem 3.3) that the inverse map is sequentially continuous, in the sense that if $\left(\varrho_{n}\right)$ is a sequence of densities satisfying $\prod^{N} \varrho_{n} \rightarrow \prod^{N} \varrho$ strongly in 
$L^{1}\left(e^{-W} d^{N} x\right)$ and weakly in $L^{1}\left(d^{N} x\right)$, and $\int W_{+} \prod^{N} \varrho_{n} d^{N} x \rightarrow \int W_{+} \prod^{N} \varrho d^{N} x$, then the corresponding sequence $\left(U_{n}\right)$ of external potentials, defined by $\varrho_{n}=\varrho\left(U_{n}\right)$, satisfies $\prod^{N} \exp \left(-U_{n}\right) \rightarrow \prod^{N} \exp (-U)$ strongly $L^{1}\left(e^{-W} d^{N} x\right)$, where $U$ is the potential such that $\varrho=\varrho(U)$.

In Sect. 4, it was shown (corollary to Lemma 4.2) that for a bounded interaction, $W \in L^{\infty}\left(d^{N} x\right)$, the inverse map $V(\varrho)$ possesses a Fréchet derivative. This derivative, at the density $\varrho_{0}$, is given by

$$
\left.\frac{\delta V}{\delta \varrho}\right|_{\varrho_{0}, V\left(\varrho_{0}\right)}=\left[\left.\frac{\delta Q}{\delta V}\right|_{\varrho_{0}, V\left(\varrho_{0}\right)}\right]^{-1}\left[\left.\frac{\delta Q}{\delta \varrho}\right|_{\varrho_{0}, V\left(\varrho_{0}\right)}\right],
$$

where

$$
Q(\varrho, V)=e^{-V} \int\left(e^{-W}\right)_{\text {sym }} \prod^{N-1}(\varrho / N) e^{-V} d^{N-1} x
$$

The inverse problem for several species of particles was treated in Sect. 5. Given $A \leqq N$ particle species with $n_{a}$ particles of each type $\left(\sum_{a=1}^{A} n_{a}=N\right)$, and an interaction $W$ such that $W_{+}$and $e^{-W}$ are in $L^{1}\left[\prod_{a=1}^{A} \prod_{a_{l}=1}^{n_{a}} \varrho_{a}\left(x_{a_{l}}\right) d x_{a_{l}}\right]$, it was shown (Theorem 5.1) that, up to constants, there exists a unique family of functions $\mathbf{V}=\left(V_{a}: \Lambda \rightarrow \mathbb{R} \mid a=1, \ldots, A\right)$ such that, for every $a, \varrho_{a}(x)=\varrho_{\mathbf{v}, a}(x)$. Moreover, each $V_{a}$ satisfies $V_{a} \in L^{1}\left(\varrho_{a} d x\right)$ and, modulo constants, $\mathbf{V}=\left(V_{a}\right)$ is the unique family which maximizes the functional $\mathfrak{F}_{A}(\mathbf{V})=\exp \left(\sum_{a=1}^{A} \int V_{a} \varrho_{a} d x\right) / Z(\mathbf{V})$. The external potentials $\mathbf{U}=\left(U_{a}\right)$ such that $\varrho_{a}(x)=\varrho_{\mathbf{U}, a}(x)$ are given by $U_{a}=-\log \left(\varrho_{a} / n_{a}\right)+V_{a}$.

The inverse problem for charge densities in systems with charged coupling was investigated in Sect. 6. These systems are characterized by an interaction $W\left(x_{1}, \ldots, x_{N}\right)$ and a fixed set of charges $q_{1}, \ldots, q_{N}$. A necessary condition for the solution of the inverse problem in the systems under consideration is the absence of total charge separation. Hence we require $Q_{-}>\int C_{-}(x) d x$ unless $Q_{-}=0$, where $Q_{-}$is the magnitude of the sum of the negative charges and $C_{-}(x)$ is the negative part of the given charge density $C(x)$.

Given a $C \in L^{1}(d x)$ with $\int C(x) d x=\sum^{N} q_{i}$ which satisfies the above condition, it is shown that, under two additional (rather technical) assumptions, the inverse problem possesses a unique solution. Here, for simplicity, we state conditions that are somewhat stronger than those required in Sect. 6. For example, if $\int|C(x)| \log |C(x)| d x<\infty$ and $W_{+} \in L^{1}\left(\prod_{i=1}^{N}\left|C\left(x_{i}\right)\right| d x_{i}\right)$ then, up to a constant, there is a unique $U$ in the class of all $[d x]$-measurable functions such that $C(x)=C_{U}(x)$ (Theorem 6.5). Furthermore, this $U$ satisfies $U \in L^{1}(|C| d x)$ and is, modulo a constant, the unique $U$ which maximizes the functional $\mathfrak{F}_{C}(U)$ $=\exp \left(-\int C \varrho d x\right) / Z(U)$ (Theorems 6.3 and 6.4). 


\section{The Inverse Problem for the Grand Canonical Ensemble}

(i) Statement of the Problem. For a given system, it is often the case that states with different numbers (and types) of particles are physically accessible. Such systems are described within the framework of the grand canonical distribution. Here we formulate the inverse problem for these systems.

The coordinates of a single particle again are taken to lie in some ( $\sigma$-finite) measure space $\langle\Lambda, d x\rangle$. For each particle number, $N=1,2, \ldots$, an interaction $W_{N}: \Lambda^{N} \rightarrow \mathbb{R}$ is specified. The generalization to several particle species is straightforward and is postponed until a later subsection.

We make no specific assumptions about the symmetry of the $W_{N}$. Moreover, for $N^{\prime} \neq N, W_{N}$ and $W_{N^{\prime}}$ need not be related in any way; each $W_{N}$ may be specified independently. (In particular, we do not assume, as is often done, that the interactions are exclusively two-body, for which it is required that $W_{N}=\sum_{i<j}^{N} W_{2}\left(x_{i}, x_{j}\right)$

If an external potential $U: \Lambda \rightarrow \mathbb{R}$ is applied to the system, the $N$-particle (canonical) partition functions $Z_{N, U}$ are given by Eq. (1.2) with $W$ replaced by $W_{N}$. The grand canonical partition function of the system is

$$
\Xi_{U}=\sum_{N=0}^{\infty} \frac{1}{N !} Z_{N, U}
$$

Remarks. (1) The coefficient of $Z_{N, U}$ often includes a factor of $e^{N \mu}$, where $\mu$ is the chemical potential. For notational convenience, we absorb this factor into the definition of $W_{N}$.

(2) The first term in the series, $Z_{0, U} \equiv Z_{0}$, is the zero-particle partition function, which is of course a constant. Here we shall assume that our systems admit the zero-particle state $\left(\Rightarrow Z_{0}>0\right)$, in which case it is traditional to take $Z_{0}=1$. We make this assumption for clarity of exposition in our subsequent proofs. However, if one wishes to consider systems which do not admit the zeroparticle state, this assumption may easily be relaxed provided that the desired expected particle number is strictly greater than one and that for some $N>n, W_{N}$

satisfies condition (1.4).
The (canonical) density for the $N$-particle state is $\varrho_{N}(U ; x)=\sum_{i=1}^{N} \varrho_{N, i}(U ; x)$, where $\varrho_{N, i}(U ; x)$ is given by Eqs. (1.1) and (1.2), with $W$ replaced by $W_{N}$. The particle density in the grand canonical ensemble is defined by

where

$$
\varrho(U ; x)=\sum_{N=1}^{\infty} \xi_{N, U} \varrho_{N}(U ; x),
$$

$$
\xi_{N, U}=\Xi_{U}^{-1}\left(Z_{N, U} / N !\right)
$$

is the probability of the $N$-particle state. The expected number of particles in the system is

$$
\int \varrho(U ; x) d x=\sum_{N=1}^{\infty} \xi_{N, U} N \equiv n .
$$

In (8.1)-(8.4), we have tacitly assumed that all expressions are well defined. 
Given an $L^{1}$ function $\varrho(x)$, the inverse problem for the grand canonical ensemble is to determine whether there exists a unique external potential, $U(x)$, such that $\varrho(x)=\varrho(U ; x)$ with $\varrho(U ; x)$ given by $(8.3)$. This problem is treated in subsection (ii). In later subsections, we examine the grand canonical analogs of the problems treated in Sects. 3-6.

The conditions under which the inverse problem for the canonical ensemble possesses a solution are summarized in Sect. 7. For the single species case, the stability condition which we imposed was finiteness of the partition function at $V=0$, i.e. $Z(0)=\int e^{-W} \prod^{N} \varrho<\infty$.

We encounter a new feature in the grand canonical ensemble. In general, an infinite number of terms contribute to the partition function $\Xi_{U}$. Thus, merely requiring that each $N$-particle state exists does not guarantee overall stability of the system. Indeed, as illustrated by the following example, there are sequences of perfectly well-behaved potentials $W_{N}$ which characterize catastrophically unstable systems in the grand canonical distribution.

Example. Suppose $\exp \left(-W_{N}\right)=N ! / N^{3}$. (Note that $W_{N}$ is constant and hence such a problem is trivial in the context of the canonical ensemble!) It is easy to see that any admissible potential $U(x)$ satisfies $e^{-U} \in L^{1}(d x)$. However, note that if $\left\|e^{-U}\right\|_{1(d x)}>1$, then the expected number of particles is infinite, while if $\left\|e^{-U}\right\|_{1(d x)} \leqq 1$, the expected number of particles is bounded by $n_{\max }=\zeta(2) /[1+\zeta(3)] \approx 0.747$. Here $\zeta$ is the Riemann zeta function. Thus, under the action of any external potential, it is impossible to have an average of as much as a single particle in this system.

In order to exclude the type of pathology illustrated above, it is clear that we must impose some condition which guarantees that our system is stable at expected particle number $n$. This condition is most easily formulated in terms of the "potential" $V$, defined by

$$
U=-\log (\varrho / n)+V
$$

where $U$ is the true external potential. Let us denote by $Z_{N}(V), \Xi(V)$, and $\varrho_{V}(x)$ the $N$-particle canonical partition function, grand canonical partition function and grand canonical density for the external potential (8.5).

The stability condition which we shall impose is

$$
\Xi(c)<\infty \text { for every } c \in \mathbb{R} \text {. }
$$

Here, $\Xi(c)$ is shorthand notation for $\Xi(V)$ with $V=$ const $\equiv c$.

Condition (8.6) is in some sense the analog of stability condition (1.5) for the canonical ensemble, since the latter is equivalent to $Z(c)<\infty$ for every $c \in \mathbb{R}$. Here, of course, $\Xi(0)<\infty$ is a much weaker condition than (8.6) and admits counterexamples of the type given above.

It is worth noting that condition (8.6) is directly implied by the statement that $Z_{N}(0) \leqq e^{N F}$ for some constant $F \in \mathbb{R}$. The latter (and stronger) condition is of course satisfied by any system which has a thermodynamic limit.

The second condition which we impose to prove existence of the inverse map is in fact significantly weaker than the analogous condition for the canonical 
ensemble. We assume that there exists a single $\tilde{N}>n$ such that $W_{\tilde{N}}$ satisfies condition (1.4). We emphasize that this condition does not exclude systems for which, if $N>\tilde{N}$, (i) $W_{N}=\infty$ on sets of positive measure (hard core stability), or (ii) $W_{N} \equiv \infty$ (truncation of the partition function $\Xi$ ). We note, however, that sufficient conditions for the true hard core case (i.e., when $W_{N}$ is hard core for every $N \geqq 2$ ) remains an open question.

(ii) Existence and Uniqueness. In this subsection, we establish that the (single species) inverse problem possesses a unique solution.

Given a non-negative function $\varrho: \Lambda \rightarrow \mathbb{R}$ with $\int_{\Lambda} \varrho(x) d x=n<\infty$, we introduce the unit measure

$$
d \mu=[\varrho(x) / n] d x
$$

on $\Lambda$, and, for each $N$, the product measure $d^{N} \mu$ on $\Lambda^{N}$.

We again use a variational technique. The relevant functional for the grand canonical ensemble is

$$
\mathfrak{G}(V)=[\Xi(V)]^{-1} \exp \left(-n \int V d \mu\right) .
$$

Formally, at least, it is easy to see that the variational derivative $\delta(\mathfrak{b} / \delta V$ vanishes if there is a $V$ such that $\varrho(x)=\varrho_{V}(x)$.

We shall consider the class of functions $\mathscr{V}_{\mathscr{G}}$ defined by

$$
\mathscr{V}_{\mathscr{G}}=\left\{V: \Lambda \rightarrow \mathbb{R} \mid V \in L^{1}(d \mu), \quad \Xi(V)<\infty\right\} .
$$

This is precisely the class of functions for which $\mathfrak{5}$ is well-defined.

We shall show that $(55$ possesses a unique maximum under the following two conditions:

(a) $\mathscr{V}_{\mathfrak{G}} \neq \emptyset$.

(b) There exists an $\tilde{N}>n$ such that $\left(W_{\tilde{N}}\right)_{+} \in L^{1}\left(d^{\tilde{N}} \mu\right)$.

Remarks. (1) As mentioned previously, we are using the convention $Z_{0}=1$. If one wishes to extend the definition of the grand canonical distribution to systems which do not admit the zero particle state, the results of this section are correct provided that $n>1$ and that the system satisfies the additional condition:

(c) There exists an $\tilde{N}^{\prime}<n$ such that $\left(W_{\tilde{N}^{\prime}}\right)_{+} \in L^{1}\left(d^{\tilde{N}^{\prime}} \mu\right)$.

(2) Although we shall show that (a) and (b) insure that $(\mathfrak{5}$ possesses a unique maximum, the example of the previous subsection indicates that these conditions are not sufficient to prove the existence of an inverse map $V(\varrho)$, i.e., if $\mathfrak{G}$ has a maximum in $\mathscr{V}_{\mathfrak{G}}$, it is not necessarily true that $\varrho_{V}=\varrho$. However, if condition $(\mathbf{a})$ is replaced by the stronger condition:

(A) For every $c \in \mathbb{R}, c \in \mathscr{V}_{\mathfrak{G}}$,

then it will be shown that an inverse map does indeed exist. Condition (A) is of course equivalent to (8.6).

(3) For future reference, we note that (b) implies that, for every $V \in \mathscr{V}_{(\mathfrak{G}}$, $\Xi(V)>Z_{0} \equiv 1$.

(4) As before, we shall find that the interacting measures

$$
d \lambda_{N}=e^{-W_{N}} d^{N} \mu
$$

play a central role. 
We note that condition (b) guarantees that at least one of the measures $d \lambda_{\tilde{N}}$ is equivalent to the underlying product measure $d^{\tilde{N}} \mu$.

We also observe that condition (a) implies that, for all $N, d \lambda_{N}$ is absolutely continuous with respect to the corresponding product measure $d^{N} \mu$. Were this not the case, then for some $N$, we would have $\exp \left(-W_{N}\right)=\infty$ on a set of nonzero $\left[d^{N} \mu\right]$ measure. However, this would mean that for every $V \in L^{1}(d \mu)$, we would have $Z_{N}(V)=\infty$; hence $\mathscr{V}_{\mathfrak{5}}=\emptyset$.

The natural measure space to consider for the grand canonical ensemble is the direct sum $\bigoplus_{N=0}^{\infty}\left\langle\Lambda^{N}, d \mu_{N} / N !\right\rangle$. The relevant space of functions is then the generalized Fock space

$$
\mathbb{L}^{p}(d \lambda)=\ell^{p}\left[\bigoplus_{N=0}^{\infty} L^{p}\left(\Lambda^{N}, d \lambda_{N} / N !\right)\right],
$$

where $L^{P}\left(\Lambda^{0}, d \lambda_{0}\right)=\mathbb{R}$. We note that this differs from the usual notion of a Fock space (even for $p=2$ ) in that the measures $d \lambda_{N}$ are not cartesian products of $d \lambda_{1}$. The only case in which $\mathbb{L}^{2}(d \lambda)$ coincides with the standard Fock space is the ideal gas.

A function $f$ in $\mathbb{L}^{p}(d \lambda)$ is a sequence of functions $f=\left(f_{N} \in L^{P}\left(\Lambda^{N}, d \lambda_{N}\right) \mid N=0,1,2, \ldots\right)$ such that

$$
\|f\|_{p(d \lambda)} \equiv\left[\left|f_{0}\right|^{p}+\sum_{N=1}^{\infty} \frac{1}{N !}\left\|f_{N}\right\|_{p\left(d \lambda_{N}\right)}^{p}\right]^{(1 / p)}<\infty .
$$

Of particular interest for the inverse problem is the product sector of $\mathbb{L}^{p}(d \lambda)$, i.e. functions $f$ such that, for all $N, f_{N}$ is a product function. In this subsection, we are especially concerned with products in which all factors are identical. For such functions, we introduce the notation

$$
\pi(g)=\left(1, g(x), \ldots, \prod_{i=1}^{N} g\left(x_{i}\right), \ldots\right) .
$$

Then, the $\mathbb{L}^{1}(d \lambda)$ norm of the function $\pi\left(e^{-V}\right)$ is simply $\Xi(V)$.

We begin the proof of existence by showing that $\mathfrak{H}_{\mathfrak{5}}$ is bounded in $\mathscr{V}_{\mathfrak{G}}$.

Proposition 8.1. Under conditions (a) and (b), 65 is (uniformly) bounded above in $\mathscr{V}_{\mathfrak{6}}$.

Proof. Let $V \in \mathscr{V}_{\mathfrak{G}}$. Then

$$
\begin{aligned}
(\mathfrak{G}(V) & \leqq \exp \left(-n \int V d \mu\right) /\left[1+Z_{\tilde{N}}(V) /(\tilde{N} !)\right] \\
& \leqq \max \left\{1,(\tilde{N} !) \exp \left\|\left(W_{\tilde{N}}\right)_{+}\right\|_{1\left(d \tilde{N}_{\mu}\right)}\right\}
\end{aligned}
$$

by Jensen's inequality.

Remark. Recall that the functional $\mathfrak{F}$ for the canonical ensemble satisfied $\mathfrak{F}(V+c)=\mathfrak{F}(V)$ for any constant $c \in \mathbb{R}$. The functional $\mathbb{5}$ does not have this property, which is of course a reflection of the fact that the zero of the potential is of physical significance in the grand canonical ensemble.

For $V \in \mathscr{V}_{\mathfrak{G} \text {, }}$, so that in particular $V \in L^{1}(d \mu)$, let us express $V$ in the form

$$
V(x)=H(x)-\log y
$$


with $\int H d \mu=0$. This decomposition, $e^{-V}=y e^{-H}$, may be regarded as a division of the function $e^{-V}$ into a potential term, $e^{-H}$, and an applied fugacity, $y$, which controls the particle number.

In this framework, condition (A) is equivalent to the statement that at $H \equiv 0$ the system is stable for all applied fugacities.

Proposition 8.2. Suppose conditions (a) and (b) are satisfied. Then any maximizing sequence, $\left(V_{k} \in \mathscr{V}_{\mathfrak{G}}\right)$, for $\left(\mathfrak{5}\right.$ has the property that the corresponding fugacities, $\left(y_{k}\right)$ defined by (8.15), are uniformly bounded away from zero and infinity.

Proof. Let $R$ denote

$$
R=\sup _{V \in \mathscr{V}_{\mathfrak{G}}}(\mathfrak{H}(V)>0,
$$

and let $\left(V_{k}\right)$ be a maximizing sequence for $\boldsymbol{6}^{5}$ in $\mathscr{V}_{\mathfrak{F}}$. Since $V_{k} \in L^{1}(d \mu)$, we have $0<y_{k}=\exp \left(-\int V_{k} d \mu\right)<\infty$ for every $k$. To show that the bound is uniform, we note that eventually $\mathfrak{5}\left(V_{k}\right) \geqq R / 2$, so that the corresponding $y_{k}$ satisfy

$$
\begin{aligned}
R / 2 \leqq \mathfrak{G}\left(V_{k}\right) & \leqq y_{k}^{n} /\left[1+y_{k}^{\tilde{N}} Z_{\tilde{N}}\left(H_{k}\right) /(\tilde{N} !)\right] \\
& \leqq \min \left\{y_{k}^{n}, y_{k}^{n-\tilde{N}}(\tilde{N} !) \exp \left\|\left(W_{\tilde{N}}\right)_{+}\right\|_{1\left(d^{\tilde{N}} \mu\right)}\right\}
\end{aligned}
$$

by Jensen's inequality. Since $\tilde{N}>n>0$, it is clear that the inequality in (8.17) is violated if the $y_{k}$ get too large or too small.

Proposition 8.3. Suppose conditions (a) and (b) are satisfied. Then, for each maximizing sequence $\left(V_{k} \in \mathscr{V}_{\mathfrak{5}}\right)$ for $\mathfrak{6}$, there is a subsequence, again denoted by $\left(V_{k}\right)$, such that $\lim _{k \rightarrow \infty} \Xi\left(V_{k}\right) \equiv \Xi_{0}$ with $0<\Xi_{0}<\infty$.

Moreover, there is a measurable function $V: \Lambda \rightarrow \mathbb{R}$ such that

$$
\pi\left(e^{-V_{k} / 2}\right) \rightarrow \pi\left(e^{-V / 2}\right) \text { weakly in } \mathbb{L}^{2}(d \lambda) .
$$

Proof. Let $\left(V_{k}\right)$ be a maximizing sequence in $\mathscr{V}_{\mathfrak{G}}$ for $\mathfrak{5}$. By Proposition 8.2 , the corresponding fugacities $\left(y_{k}\right)$ are uniformly bounded away from zero and infinity. Since $\left(\mathfrak{5}\left(V_{k}\right)=y_{k}^{n} / \Xi\left(V_{k}\right) \leqq R\right.$, it follows immediately that $\Xi\left(V_{k}\right)$ is uniformly bounded above and below. Hence there is a subsequence, which we shall again denote by $\left(V_{k}\right)$, such that $\Xi\left(V_{k}\right)$ converges to a finite nonzero constant, $\Xi_{0}$.

Next, consider the sequence of functions $\pi\left(e^{-V_{k} / 2}\right)$. Since $\left\|\left(e^{-V_{k} / 2}\right)\right\|_{2(d \lambda)}^{2}=\Xi\left(V_{k}\right)$, the sequence is norm-bounded and thus, by the Banach-Alaoglu theorem, it has a weak limit in $\mathbb{L}^{2}(d \lambda)$. It is, in fact, nontrivial to establish that the weak limit is a product function. However, as proved in Theorem A.6, it is sufficient that one of the measures, here $d \lambda_{\tilde{N}}$, be equivalent to the underlying product measure $d^{\tilde{N}} \mu$, and that all the other measures be absolutely continuous with respect to their corresponding product measures. As remarked earlier, these conditions are satisfied and thus the weak limit is of the asserted form.

Theorem 8.4. Under conditions (a) and (b), there exists a $V \in \mathscr{V}_{\mathfrak{G}}$, which maximizes $\mathbb{6}$.

Proof. Let $\left(V_{k}\right)$ be a maximizing sequence for $(\mathfrak{5}$ with an associated weak limit, $V$, as given by Eq. (8.18). 
Let us verify that $V \in \mathscr{V}_{\mathfrak{G}}$. By the weak $\mathbb{L}^{2}(d \lambda)$ convergence of $\pi\left(e^{-V_{k} / 2}\right)$, we have

$$
\begin{aligned}
\Xi_{0} & =\lim _{k \rightarrow \infty} \Xi\left(V_{k}\right)=\lim _{k \rightarrow \infty}\left\|\pi\left(e^{-V_{k} / 2}\right)\right\|_{2(d \lambda)}^{2} \\
& \geqq\left\|\pi\left(e^{-V / 2}\right)\right\|_{2(d \lambda)}^{2}=\Xi(V) .
\end{aligned}
$$

Thus $\Xi(V)<\infty$.

The remainder of this proof is essentially the same as the corresponding arguments for the canonical ensemble (cf. Theorem 2.2).

Since $Z_{\tilde{N}}(V)<\infty$ and $\left(W_{\tilde{N}}\right)_{+} \in L^{1}\left(d^{\tilde{N}} \mu\right)$, reasoning along the lines of Eqs. (2.19) and (2.20) establishes that $V_{-} \in L^{1}(d \mu)$.

Next, a derivation identical to that of Eqs. (2.21)-(2.28) with $W$ replaced by $W_{\tilde{N}}$ proves that

$$
R \Xi_{0}=\lim _{k \rightarrow \infty} \exp \left(-n \int V_{k} d \mu\right) \leqq \exp \left(-n \int V d \mu\right)
$$

This inequality shows that $V_{+} \in L^{1}(d \mu)$ and hence $V \in \mathscr{V}_{\mathfrak{F}}$.

Moreover, combining (8.19) and (8.20), we have

$$
\mathfrak{G}(V) \geqq \lim _{k \rightarrow \infty} \mathfrak{G}\left(V_{k}\right)=\sup _{V \in \mathscr{V}_{\mathfrak{G}}} \mathfrak{G}(V) .
$$

Corollary. If $\left(V_{k} \in \mathscr{V}_{\mathfrak{G}}\right)$ is any maximizing sequence for $(\mathfrak{5}$, then there is a subsequence such that the functions $\pi\left(e^{-V_{k} / 2}\right)$ converge strongly in $\mathbb{L}^{2}(d \lambda)$.

Proof. Weak convergence of a subsequence was established in Proposition 8.3. Thus it suffices to prove convergence of the norm, i.e. $\lim _{k \rightarrow \infty} \Xi\left(V_{k}\right)=\Xi(V)$. If this convergence failed, we should have $\lim _{k \rightarrow \infty} \Xi\left(V_{k}\right)>\Xi(V)$, which implies that $R<\mathfrak{b}(V)$, a contradiction.

Theorem 8.5. Under conditions (a) and (b), the $V$ which maximizes $\mathbb{5}$ is unique a.e. $[d \mu]$ in $\mathscr{V}_{\mathfrak{G}}$.

Proof. Suppose there exists $V_{1}, V_{2} \in \mathscr{V}_{\mathfrak{G}}$ such that $\mathbb{6}\left(V_{1}\right)=\mathfrak{G}\left(V_{2}\right)=\sup _{V \in \mathscr{V}_{\mathfrak{G}}} \mathfrak{6}(V)$. Then, using Cauchy-Schwarz in a manner entirely analogous to the proof of Proposition 6.4 , we conclude that the inequality

$$
\Xi\left[(1 / 2)\left(V_{1}+V_{2}\right)\right] \leqq \sqrt{\Xi\left(V_{1}\right)} \sqrt{\Xi\left(V_{2}\right)}
$$

must be saturated. This implies that

$$
\pi\left(e^{-V_{1} / 2}\right)=(\text { const }) \pi\left(e^{-V_{2} / 2}\right) \text { a.e. }[d \lambda] .
$$

However, for products of identical factors, the only consistent choice for the scale factor is 1 . Thus (8.23), and equivalence of $d \lambda_{\tilde{N}}$ and $d^{\tilde{N}} \mu$, imply that $V_{1}(x)=V_{2}(x)$ a.e. $[d \mu]$.

Corollary. If $\left(V_{k}\right)$ is any maximizing sequence for $\mathbb{5}$ in $\mathscr{V}_{\mathfrak{F}}$, then

$$
\pi\left(e^{-V_{k} / 2}\right) \rightarrow \pi\left(e^{-V / 2}\right) \text { strongly in } \mathbb{L}^{2}(d \lambda),
$$

where $V$ is the maximizing potential. 
Proof. This follows immediately from the strong convergence of any subsequence and uniqueness.

Remark. We have seen that conditions (a) and (b) are sufficient to prove the existence of a unique maximizer for the functional $\mathbf{6}$. However, these conditions alone are not enough to prove that $\left(55\right.$ has a functional derivative in $\mathscr{V}_{\mathfrak{6}}$, and hence that the maximizing $V$ satisfies $\varrho=\varrho_{V}$.

In fact, without any additional assumptions, we can show that 05 has a "onesided" functional derivative in $\mathscr{V}_{\mathfrak{G}}$, which directly implies the variational inequality $\varrho_{V} \leqq \varrho$. Thus if $\langle N\rangle_{V}=\int \varrho_{V}(x) d x$ is the expected number of particles in the state with external potential $U=-\log (\varrho / n)+V$, conditions (a) and (b) only imply that $\langle N\rangle_{V} \leqq n$. This is precisely the difficulty encountered in the example in subsection (i), for which the system (at any external potential) could not support $n \gtrsim 0.747$ particles.

In the following lemma and proposition, it is shown that, at the maximizing potential, systems which satisfy condition (A) always have the correct number of particles.

Lemma 8.6. Suppose condition (A) is satisfied. Let $V \in \mathscr{V}_{\mathfrak{F}}$ and $\varepsilon>0$, and define

$$
V_{\varepsilon}=V+\varepsilon^{2} V_{-}
$$

where $V_{-}$is the negative part of $V$. Then

(1) For every $\sigma \in \mathbb{R},\left(V_{\varepsilon}+\sigma \varepsilon\right) \in \mathscr{V}_{\mathfrak{G}}$.

(2) $\mathfrak{G}\left(V_{\varepsilon}\right) \geqq \mathfrak{G}(V)\left[1+O\left(\varepsilon^{2}\right)\right]$.

(3) $\varepsilon\left[\langle N\rangle_{V}-\langle N\rangle_{V_{\varepsilon}}\right]=o(\varepsilon)$.

Proof. (1) First we note that if $V_{1}(x) \geqq V_{2}(x)$, then $\Xi\left(V_{1}\right) \leqq \Xi\left(V_{2}\right)$. Also observe that, by Hölder's inequality, $\Xi(V)$ is log convex, i.e.

$$
\Xi\left(\lambda V_{1}+(1-\lambda) V_{2}\right) \leqq \Xi\left(V_{1}\right)^{\lambda} \Xi\left(V_{2}\right)^{1-\lambda}
$$

for any $V_{1}, V_{2}$, and $\lambda \in[0,1]$.

Let $V \in \mathscr{V}_{\mathfrak{G}}, \varepsilon>0$, and $\sigma \in \mathbb{R}$. Define $V_{\varepsilon}$ as in (8.25). Clearly $\left(V_{\varepsilon}+\sigma \varepsilon\right) \in L^{1}(d \mu)$, and hence it suffices to show that $\Xi\left(V_{\varepsilon}+\sigma \varepsilon\right)<\infty$. Noting that $V_{\varepsilon} \geqq\left(1-\varepsilon^{2}\right) V$, and using log convexity, we have

$$
\Xi\left(V_{\varepsilon}+\sigma \varepsilon\right) \leqq \Xi\left(\left(1-\varepsilon^{2}\right) V+\sigma \varepsilon\right) \leqq \Xi(V)^{1-\varepsilon^{2}} \Xi\left(\frac{\sigma}{\varepsilon}\right)^{\varepsilon^{2}},
$$

which is finite by condition (A).

(2) Let us express $V_{\varepsilon}$ in the form $V_{\varepsilon}=\left(1-\varepsilon^{2}\right) V+\varepsilon^{2} V_{+}$, where $V_{+}$is the positive part of $V$. Then, using the log convexity of $\Xi$, we have

$$
\mathfrak{5}\left(V_{\varepsilon}\right) \geqq \exp \left[-\int \varrho V-\varepsilon^{2} \int \varrho V_{-}\right] / \Xi(V)^{1-\varepsilon^{2}} \Xi\left(V_{+}\right)^{\varepsilon^{2}}=\mathfrak{5}(V)\left[1+O\left(\varepsilon^{2}\right)\right] .
$$

(3) For any $V,\langle N\rangle_{V}$ may be written in the form

$$
\langle N\rangle_{V}=\left[\sum_{N=1}^{\infty} N Z_{N}(V) / N !\right] /\left[\sum_{N=0}^{\infty} Z_{N}(V) / N !\right] .
$$

By monotone convergence, the numerator and denominator of $\langle N\rangle_{V_{\varepsilon}}$ (separately) converge to the numerator and denominator of $\langle N\rangle_{V}$ as $\varepsilon \rightarrow 0$. Thus $\varepsilon\left[\langle N\rangle_{V}-\langle N\rangle_{V_{\varepsilon}}\right]=o(\varepsilon)$. 
Proposition 8.7. Suppose condition (A) is satisfied. Then for every $V \in \mathscr{V}_{\tilde{\sigma}}$, either

(1) $\langle N\rangle_{V}=n$, or

(2) there exists $\bar{V} \in \mathscr{V}_{\mathfrak{G}}$ such that $\mathfrak{5}(\bar{V})>\mathfrak{G}(V)$.

Proof. Let $V \in \mathscr{V}_{\tilde{F}}$. If $\langle N\rangle_{V} \neq n$, define

$$
\sigma_{V}=\left\{\begin{array}{lll}
+1 & \text { if } & \langle N\rangle_{V}>n \\
-1 & \text { if } & \langle N\rangle_{V}<n
\end{array} .\right.
$$

Then by part (1) of Lemma 8.6, $\left(V_{\varepsilon}+\sigma_{V} \varepsilon\right) \in \mathscr{V}_{\mathfrak{G}}$. We have

$$
\begin{aligned}
\mathfrak{5}\left(V_{\varepsilon}+\sigma_{V} \varepsilon\right) & =\mathfrak{G}\left(V_{\varepsilon}\right)\left[1+\sigma_{V} \varepsilon\left(\langle N\rangle_{V_{\varepsilon}}-n\right)\right]+O\left(\varepsilon^{2}\right) \\
& \geqq \mathfrak{b}(V)\left[1+\varepsilon\left|\langle N\rangle_{V}-n\right|\right]+o(\varepsilon),
\end{aligned}
$$

where the second step follows from parts (2) and (3) of Lemma 8.6. However, (8.31) implies that if $\langle N\rangle_{V} \neq n$, then for $\varepsilon$ sufficiently small, $\left(\mathfrak{5}\left(V_{\varepsilon}+\sigma_{V} \varepsilon\right)>\mathfrak{5}(V)\right.$.

Theorem 8.8. Suppose conditions (A) and (b) are satisfied. Then there exists a $V \in \mathscr{V}_{\mathfrak{G}}$ such that $\varrho(x)=\varrho_{V}(x)$.

Proof. First recall (cf. remark following Theorem 2.3) that it is sufficient to demonstrate the existence of a $V \in \mathscr{V}_{0 \mathfrak{5}}$ such that $\varrho(x)=\varrho_{V}(x)$ a.e. $[d x]$.

Now by Theorem 8.4, there exists a $V \in \mathscr{V}_{\mathfrak{G}}$ which maximizes $V$. For this $V$, calculate $\varrho_{V}$ and define $T=\left\{x \in \Lambda \mid \varrho_{V}(x)>\varrho(x)\right\}$. Since $\chi_{T}$, the characteristic function of the set $T$, is a nonnegative $L^{\infty}$ function, it is clear that $\left(V+\varepsilon \chi_{T}\right) \in \mathscr{V}_{\mathfrak{G}}$ for all $\varepsilon \geqq 0$. Thus

$$
0 \geqq \limsup _{\varepsilon \rightarrow 0} \varepsilon^{-1}\left[\mathfrak{5}\left(V+\varepsilon \chi_{T}\right)-\mathfrak{G}(V)\right]=\mathfrak{G}(V) \int_{T}\left|\varrho_{V}-\varrho\right| d x,
$$

which implies that $\varrho_{V}(x) \leqq \varrho(x)$ a.e. $[d x]$.

However, since $V$ is the maximizing potential, Proposition 8.7 implies that $\int \varrho_{V}(x) d x=n=\int \varrho(x) d x$. Thus $\varrho_{V}(x)=\varrho(x)$ a.e. $[d x]$.

Theorem 8.9. Under conditions (A) and (b), there exists a unique $V: \Lambda \rightarrow \mathbb{R}$ in the class of all $[d \mu]$-measurable functions such that $\varrho(x)=\varrho_{V}(x)$.

Proof. First, we show that there is a unique $V \in \mathscr{V}_{\mathfrak{G}}$ such that $\varrho(x)=\varrho_{V}(x)$. By Theorem 8.8, the $V$ which maximizes $\mathfrak{G}^{2}$ satisfies $\varrho=\varrho_{V}$, and, by Theorem 8.5 , the maximizing $V$ is unique a.e. $[d \mu]$. In order to prove that there are no other $V \in \mathscr{V}_{\mathfrak{G}}$ such that $\varrho=\varrho_{V}$, we must show that $\mathfrak{5}$ has no other extrema. This follows immediately from the fact that $\mathbb{6}$ is strictly log concave in $V$.

Next, we prove that any measurable function $V$ which satisfies $\varrho=\varrho_{V}$ must be in $\mathscr{V}_{\mathfrak{G}}$. If $\varrho=\varrho_{V}$, then evidently

$$
Z_{0}<\Xi(V)<\infty
$$

The upper bound immediately implies that $Z_{\tilde{N}}(V)<\infty$. We claim that the lower bound implies that $Z_{\tilde{N}}(V)>0$. In fact, suppose $Z_{\tilde{N}}(V)=0$. Then, since $d \lambda_{\tilde{N}}$ and $d^{\tilde{N}} \mu$ are equivalent, it follows that $\sum_{i=1}^{N} V\left(x_{i}\right)=\infty$ a.e. $\left[d^{\tilde{N}} \mu\right]$. However, this implies $V(x)=\infty$ a.e. $[d \mu]$, from which we conclude that $Z_{N}(V)=0$ for every $N>0$. Thus 
$\Xi(V)=Z_{0}$, a contradiction. Hence we have

$$
0<Z_{\tilde{N}}(V)<\infty .
$$

Using an argument along the lines of the proof of Theorem 2.4(a), $Z_{\tilde{N}}(V)<\infty$ implies $V_{-} \in L^{1}(d \mu)$. Similarly, noting that the hypothesis $\mathscr{V}_{\mathfrak{W}} \neq \emptyset$ means that there exists a $V_{0}$ with $V_{0} \in L^{1}(d \mu)$ and $Z_{\tilde{N}}\left(V_{0}\right)>0$, the lower bound $Z_{\tilde{N}}(V)>0$ implies that $V_{+} \in L^{1}(d \mu)$.

Thus $V \in \mathscr{V}_{\mathfrak{F}}$ as asserted.

(iii) Continuity of the Inverse Map. In Sect. 3, we proved that the inverse map for the canonical ensemble is sequentially continuous. Here we prove an analogous result for the grand canonical ensemble.

Given a system characterized by some family of interactions $W_{N}: \Lambda^{N} \rightarrow \mathbb{R}$, $N=1,2, \ldots$, we shall say that a nonnegative function $\varrho \in L^{1}(d x)$ is an admissible density if conditions (A) and (b) of subsection (ii) are satisfied.

By condition (A), if $\varrho$ is admissible then $\pi\left[(\varrho / n) e^{-c}\right] \in \mathbb{L}^{1}(d \sigma)$ for every $c \in \mathbb{R}$, where the generalized Fock space $\mathbb{L}^{1}(d \sigma)$ is defined as in Eq. (8.11) with the measures $d \lambda_{N}$ replaced by the measures

$$
d \sigma_{N}=e^{-W_{N}} d^{N} x
$$

Condition (b) says that there must exist some $\tilde{N}>n$ such that $\int\left(W_{\tilde{N}}\right)_{+} \prod^{\tilde{N}}(\varrho / n) d^{\tilde{N}} x<\infty$. Let us consider the space of all admissible densities which satisfy (b) for a fixed (common) value $\tilde{N}$ :

$$
\begin{gathered}
\mathscr{D}=\left\{\varrho: \Lambda \rightarrow \mathbb{R}^{+} \mid \varrho \in L^{1}(d x), \forall c \in \mathbb{R} \quad \pi\left[(\varrho / n) e^{-c}\right] \in \mathbb{L}^{1}(d \sigma),\right. \\
\left.\int\left(W_{\tilde{N}}\right)_{+} \prod^{\tilde{N}}(\varrho / n) d^{\tilde{N}} x<\infty, \tilde{N}>\int \varrho d x=n\right\} .
\end{gathered}
$$

Reference to $\tilde{N}$ will be suppressed in our notation.

Our definition of convergence in $\mathscr{D}$ is analogous to that of Sect. 3. In particular, we say that a sequence $\left(\varrho_{k} \in \mathscr{D}\right)$ converges to $\varrho \in \mathscr{D}$ if

$$
\pi\left(\varrho_{k} / n_{k}\right) \rightarrow \pi(\varrho / n) \quad \text { weakly in } \mathbb{L}^{1}(d \sigma)
$$

and

$$
\prod^{\tilde{N}}\left(\varrho_{k} / n_{k}\right) \rightarrow \prod^{\tilde{N}}(\varrho / n) \quad \text { strongly in } L^{1}\left(d \sigma_{\tilde{N}}\right)
$$

and

$$
\varrho_{k} \rightarrow \varrho \quad \text { weakly in } L^{1}(d x)
$$

and

$$
\int\left(W_{\tilde{N}}\right)_{+} \prod^{\tilde{N}}\left(\varrho_{k} / n_{k}\right) d^{\tilde{N}} x \rightarrow \int\left(W_{\tilde{N}}\right)_{+} \prod^{\tilde{N}}(\varrho / n) d^{\tilde{N}} x
$$

and, for every $c \in \mathbb{R}$,

$$
\underset{k \rightarrow \infty}{\limsup } \mid\left\|\pi\left[\left(\varrho_{k} / n_{k}\right) e^{-c}\right]\right\|_{1(d \sigma)}<\infty .
$$


We shall denote this type of convergence by $\varrho_{k} \rightarrow \varrho$ in $\mathscr{D}$. We note that $\varrho_{k} \rightarrow \varrho$ in $\mathscr{D}$ implies that $\varrho$ is admissible.

Remarks. (1) The above convergence is in fact weaker than the naive generalization of the convergence required for the canonical ensemble. In this case, the space $\mathbb{L}^{1}(d \sigma)$ replaces $L^{1}(d \sigma)$, and hence the generalization of the convergence in (3.5) would be that $\pi\left(\varrho_{k} / n_{k}\right) \rightarrow \pi(\varrho / n)$ strongly in $\mathbb{L}^{1}(d \sigma)$. Here, however, we only require weak convergence in $\mathbb{L}^{1}(d \sigma)$, provided that the $\tilde{N}^{\text {th }}$ term in $\pi\left(\varrho_{k} / n_{k}\right)$ converges strongly in $L^{1}\left(d \sigma_{\tilde{N}}\right)$. Likewise, we only require [Eq. (8.40)] that the $\tilde{N}^{\text {th }}$ term satisfy the analog of condition (3.7).

Thus the grand canonical inverse function is stabilized by the properties of a single potential, $W_{\hat{N}}$.

(2) As discussed previously, the condition $\Xi(c)<\infty$ for all $c \in \mathbb{R}$ guarantees that the system, at potential $V=0$, is stable for all applied fugacities. Equation (8.41) simply requires uniformity in this condition. The analog of (8.41) is trivially satisfied for the canonical ensemble.

We note that if there exists a $B \in \mathbb{R}$ such that $\int \prod^{N}\left(\varrho_{k} / n_{k}\right) d \sigma_{N} \leqq e^{B N}$ for all $k$, then condition (8.41) is satisfied.

By the results of subsection (ii), for any $\varrho \in \mathscr{D}$, there exists a unique inverse function $U=-\log (\varrho / n)+V$ satisfying $\pi\left(e^{-U}\right) \in \mathbb{L}^{1}(d \sigma)$. Let us consider this space of potentials with the strong $\mathbb{L}^{1}(d \sigma)$ topology.

It will be shown that the inverse map is sequentially continuous in the sense that if $\varrho_{k} \rightarrow \varrho$ in $\mathscr{D}$, then $\pi\left[\exp \left(-U_{k}\right)\right] \rightarrow \pi[\exp (-U)]$ strongly in $\mathbb{L}^{1}(d \sigma)$.

Notation. For a convergent sequence of densities $\varrho_{k} \rightarrow \varrho$ in $\mathscr{D}$, we shall denote by $n$, $Z_{N}, \Xi$, $\left(\mathfrak{5}\right.$, and $\mathscr{V}_{\mathfrak{G}}$ the quantities defined in subsections (i) and (ii) for the limit function $\varrho$. The maximizing potential and corresponding external potential for $\varrho$ will be denoted by $V$ and $U$.

The analogous quantities for each term in the sequence shall be indexed by $k$.

We will also use the notation

$$
R=\sup _{S \in \mathscr{V}_{\mathfrak{G}}} \mathfrak{G}(S)
$$

and denote by $R_{k}=R\left(\varrho_{k}\right)$ the corresponding quantity for $\varrho_{k}$.

Remark. Proposition 8.1 implies that $R(\varrho)$ is finite for every $\varrho \in \mathscr{D}$.

As in Sect. 3, the first step in the proof of continuity is to show that $R(\varrho)$ is sequentially lower semicontinuous. This is established in the following two lemmas.

Lemma 8.10. Suppose $\varrho_{k} \rightarrow \varrho$ in $\mathscr{D}$. Then for every function $S$ with $S_{-} \in L^{\infty}(d \mu)$, and $S(x)=0$ whenever $\varrho(x)=0$,

$$
\Xi_{k}(S) \rightarrow \Xi(S)
$$

Remark. Functions $S \in L^{\infty}(d \mu)$ with $S(x)=0$ whenever $\varrho(x)=0$ also satisfy $S \in L^{\infty}\left(d \mu_{k}\right)$ for every $k$, with $\|S\|_{\infty\left(d \mu_{k}\right)} \leqq\|S\|_{\infty(d \mu)}=\|S\|_{\infty(d x)}$.

All statements in the following proof will refer to the subset, $K$, of $\Lambda$, which is the union of the supports of all densities in the sequence. Then, since for every $N$ and $k$ the measure $\prod^{N}\left(\varrho_{k} / n_{k}\right) d \sigma_{N}$ is absolutely continuous with respect to 
$\prod^{N}\left(\varrho_{k} / n_{k}\right) d^{N} x$ [see remark in subsection (ii)], it follows that $d \sigma_{N}$ is absolutely continuous with respect to $d^{N} x$ on $K^{N}$.

Thus, for the functions $S$ under consideration, $\prod^{N} e^{-S} \in L^{\infty}\left(d \sigma_{N}\right)$ on $K^{N}$.

Note that functions of the form $\pi\left(e^{-S}\right)$ are typically not in $\mathbb{L}^{\infty}(d \sigma)$ despite the fact that, for each $N, \prod^{N} e^{-S} \in L^{\infty}\left(d \sigma_{N}\right)$. Thus this lemma does not follow directly from the assumption $\pi\left(\varrho_{k} / n_{k}\right) \rightarrow \pi(\varrho / n)$ in $\mathbb{L}^{1}(d \sigma)$.

Proof. First observe that $\pi\left(\varrho_{k} / n_{k}\right) \rightarrow \pi(\varrho / n)$ weakly in $\mathbb{L}^{1}(d \sigma)$ implies that, for each $N$,

$$
\prod^{N}\left(\varrho_{k} / n_{k}\right) \rightarrow \prod^{N}(\varrho / n) \quad \text { weakly in } L^{1}\left(d \sigma_{N}\right)
$$

Then, since any $S$ satisfying the hypotheses also satisfies $\prod^{N} e^{-S} \in L^{\infty}\left(d \sigma_{N}\right)$ on $K^{N}$, we have

$$
Z_{k}^{N}(S) \rightarrow Z^{N}(S)
$$

for every $N$.

Let $c \equiv\left\|S_{-}\right\|_{\infty}$. By $(8.41)$

$$
\sum \frac{1}{N !} e^{2 N c} Z_{k}^{N}(0) \equiv A<\infty .
$$

It follows immediately that, for every $N$,

$$
\frac{1}{N !} Z_{k}^{N}(S) \leqq \frac{e^{N c}}{N !} Z_{k}^{N}(0) \leqq A e^{-N c}
$$

Thus by dominated $\ell^{1}$ convergence,

$$
\sum \frac{1}{N !} Z_{k}^{N}(S) \rightarrow \sum \frac{1}{N !} Z^{N}(S)
$$

Lemma 8.11. Suppose $\varrho_{k} \rightarrow \varrho$ in $\mathscr{D}$. Then

$$
\liminf _{k \rightarrow \infty} R_{k} \geqq R \text {. }
$$

Proof. It is clear that a maximizing sequence for $\mathbf{5}$ may be taken among functions $S \in L^{\infty}(d \mu)$ with $S(x)=0$ whenever $\varrho(x)=0$. Then, using the results of Lemma 8.10 and Eq. (8.39), the proof of this lemma is identical to that of Lemma 3.1.

Proposition 8.12. Suppose $\varrho_{k} \rightarrow \varrho$ in $\mathscr{D}$. Then if $\left(V_{k}\right)$ is the corresponding sequence of maximizing potentials, $\Xi_{k}\left(V_{k}\right)$ is (uniformly) bounded above.

Proof. The proof of this proposition is similar to that of Propositions 8.2 and 8.3.

Lemma 8.11 shows that, eventually, $\mathfrak{G}_{k}\left(V_{k}\right) \geqq \frac{1}{2} \mathfrak{G}(V)>0$. By reasoning analogous to the proof of Proposition 8.2, we have

$$
0<\frac{1}{2} \mathfrak{G}(V) \leqq \min \left\{y_{k}^{n_{k}}, y_{k}^{\tilde{N}-n_{k}}(\tilde{N} !) \exp \left(-\int\left(W_{\tilde{N}}\right)_{+} d^{\tilde{N}} \mu_{k}\right)\right\},
$$


where $y_{k}=\exp \left(-\int V_{k} d \mu_{k}\right)<\infty$. Conditions (8.39) and (8.40) imply that $n_{k} \rightarrow n$ and $\int\left(W_{\tilde{N}}\right)_{+} d^{\tilde{N}} \mu_{k} \rightarrow \int\left(W_{\tilde{N}}\right)_{+} d^{\tilde{N}} \mu$. Thus it follows from (8.50) that the $y_{k}$ are uniformly bounded above. This of course implies a uniform upper bound on $\Xi_{k}\left(V_{k}\right)$ $=y_{k}^{n_{k}} / \mathfrak{G}_{k}\left(V_{k}\right)$.

Theorem 8.13. The inverse map is sequentially continuous in the sense that if $\varrho_{k} \rightarrow \varrho$ in $\mathscr{D}$, and if $\left(U_{k}\right)$ is the corresponding sequence of external potentials defined by $\varrho_{k}=\varrho\left(U_{k}\right)$, then

$$
\pi\left[\exp \left(-U_{k}\right)\right] \rightarrow \pi[\exp (-U)] \text { strongly in } \mathbb{L}^{1}(d \sigma),
$$

where $U$ is the potential such that $\varrho=\varrho(U)$.

Proof. The proof of this statement parallels the proofs of Proposition 3.2 and Theorem 3.3.

Let $\left(V_{k}\right)$ be the sequence of maximizing potentials for the densities $\left(\varrho_{k}\right)$. Since $\Xi_{k}\left(V_{k}\right)$ is uniformly bounded, a subsequence of the functions $\pi\left[\left(\varrho_{k} / n_{k}\right)^{1 / 2} \exp \left(-V_{k} / 2\right)\right]$ converges weakly in $\mathbb{L}^{2}(d \sigma)$. Theorem A.6 shows that the weak limit is of the form $\pi[\exp (-\hat{U} / 2)]$.

As in Eq. (3.15), we define the function $\hat{V}: \Lambda \rightarrow \mathbb{R}$ by $e^{-\hat{U}}=(\varrho / n) e^{-\hat{V}}$ whenever $\varrho>0$, and $\hat{V}=0$ when $\varrho=0$.

Clearly $\pi\left[\left(\varrho_{k} / n_{k}\right)^{1 / 2} \exp \left(-V_{k} / 2\right) \rightarrow \pi[\exp (-\hat{U} / 2)]\right.$ weakly in $\mathbb{L}^{2}(d \sigma)$ implies that $\prod^{\grave{N}}\left(\varrho_{k} / n_{k}\right)^{1 / 2} \exp \left(-V_{k} / 2\right) \rightarrow \prod^{\tilde{N}} \exp (-\hat{U} / 2)$ weakly in $L^{2}\left(d \sigma_{\tilde{N}}\right)$. Using this and condition (8.38), we easily derive the analog of the corollary to Proposition 3.2:

$$
\prod^{\tilde{N}}\left(\varrho_{k} / n_{k}\right) \exp \left(-V_{k} / 2\right) \rightarrow \prod^{\tilde{N}}(\varrho / n) \exp (-\hat{V} / 2) \text { weakly in } L^{1}\left(d \sigma_{\tilde{N}}\right) .
$$

Next, the fact that $\Xi_{k}\left(V_{k}\right)$ is uniformly bounded implies the analog of Eq. (3.19):

$$
\infty>\Xi_{0}=\lim _{k \rightarrow \infty} \Xi_{k}\left(V_{k}\right) \geqq \Xi(\hat{V}),
$$

which shows that $\hat{V}_{-} \in L^{1}(d \mu)$.

Finally, repeating the analysis of Eqs. (3.20)-(3.34) with $W$ replaced by $W_{\tilde{N}}$ yields the inequality

$$
\exp \left(-\int \hat{V} \varrho d x\right) \geqq \limsup _{k \rightarrow \infty} \exp \left(-\int V_{k} \varrho_{k} d x\right),
$$

which shows that $\hat{V} \in \mathscr{V}_{\mathfrak{G}}$ and that $\mathscr{\mathfrak { G }}(\hat{V}) \geqq \limsup _{k \rightarrow \infty} \mathfrak{G}_{k}\left(V_{k}\right)$. Then, by Lemma 8.11, $\hat{V}$ maximizes $(5)$.

An argument along the lines of Eqs. (3.37)-(3.41) establishes convergence of the original sequence $\pi\left[\left(\varrho_{k} / n_{k}\right)^{1 / 2} \exp \left(-V_{k} / 2\right)\right] \rightarrow \pi\left[(\varrho / n)^{1 / 2} \exp (-\hat{V} / 2)\right]$ strongly in $\mathbb{L}^{2}(d \sigma)$. Thus we have $\hat{V}=V$ and

$$
\pi\left[\left(\varrho_{k} / n_{k}\right) \exp \left(-V_{k}\right)\right] \rightarrow \pi[(\varrho / n) \exp (-V)] \text { strongly in } \mathbb{L}^{1}(d \sigma) .
$$

(iv) Differentiability of the Inverse Map. In this subsection, we establish conditions under which the grand canonical inverse map possesses a Fréchet derivative. Recall that in order to prove the analogous result for the canonical ensemble, we required that $W$ be bounded above and below (i.e., $W \in L^{\infty}$ ). Here we 
impose much weaker conditions on the family of interactions $\left(W_{N}\right)$. In particular, we require that each $W_{N}$ be bounded below according to

$$
W_{N}\left(x_{1}, \ldots, x_{N}\right) \geqq-B N,
$$

but we only require that a single $W_{N}, N>0$, be bounded above.

Equation (8.56) is essentially a strong version of condition (A). It is, in fact, the stability condition that is often imposed to exclude systems with catastrophic behavior (see, for example, Ruelle [10]).

The second condition is satisfied by most physical systems, because one typically has $W_{1}=0$. For convenience in the subsequent proofs we shall take $W_{1}=0$, but we emphasize that this is easily replaced by the condition $\left(W_{N}\right)_{+} \in L^{\infty}\left(d^{N} x\right)$ for some $N>0$.

It is also assumed, without future reference, that there is some $N \neq 1$ such that $e^{-W_{N}} \neq 0$. Otherwise, the problem is trivial.

It is worth pointing out that there are systems which satisfy all the above conditions, which do not satisfy condition (b) of subsection (ii) (and therefore to which our previously established results may not be applied). Notable among such systems are those with hard core interactions. Our proof of differentiability will, in fact, also establish local existence of an inverse map for any system with $W_{1}=0$ and $W_{N} \geqq-B N$.

Remark. As in Sect. 4, we find it convenient to replace each $e^{-W_{N}}$ by $\left(e^{-W_{N}}\right)_{\text {sym }}$, the symmetric part of $e^{-W_{N}}$. This can of course be done without loss of generality since $e^{-W_{N}}$ is summed over permutations in all relevant expressions, and since $W_{N} \geqq-B N$ implies that $-\log \left(e^{-W_{N}}\right)_{\text {sym }} \geqq-B N$. Henceforth, the subscript sym will be suppressed.

We shall also use the convention $U=-\log \varrho+V$, rather than $U=-\log (\varrho / n)+V$, to simplify our notation. Then the measure which appears in the partition function is

$$
d \mu=\varrho d x
$$

which, although no longer a probability measure, is still finite.

With these conventions, the definition of $\varrho_{V}$ is

$$
\varrho_{V}=[\Xi(V)]^{-1} \sum_{N=1}^{\infty} \frac{N}{N !} \int e^{-W_{N}} \prod^{N} \varrho e^{-V} d^{N-1} x .
$$

Our method of proof is very similar to that used in Sect. 4.

We first show that the inverse image, should it exist, is in $L^{\infty}(d \mu)$.

Proposition 8.14. Suppose $W_{1}=0$ and $W_{N} \geqq-B N$ for each $N$. Then, if $V$ satisfies $\varrho=\varrho_{V}, V \in L^{\infty}(d \mu)$.

Proof. If $V$ satisfies $\varrho=\varrho_{V}$, then on $\{x \mid \varrho(x)>0\}$

$$
\begin{aligned}
e^{+V(x)} & =[\Xi(V)]^{-1} \sum_{N=1}^{\infty} \frac{N}{N !} e^{-W_{N}} \prod^{N-1} \varrho e^{-V} d^{N-1} x \\
& \geqq[\Xi(V)]^{-1},
\end{aligned}
$$

since $W_{1}=0$. This implies that $V(x)$ is bounded below and, in particular, that $e^{-V} \in L^{\infty}(d \mu)$. 
Then, since $W_{N} \geqq-B N$,

$$
e^{+V(x)} \leqq[\Xi(V)]^{-1} \sum_{N=1}^{\infty} \frac{N}{N !} e^{N B}\left\|e^{-V}\right\|_{\infty(d \mu)}^{N-1},
$$

so that $V(x)$ is also bounded above on $\{x \mid \varrho(x)>0\}$.

Remark. Since the value of $V$ is undetermined whenever $\varrho=0$, we again define $V$ so that

$$
e^{+V(x)}=[\Xi(V)]^{-1} \sum_{N=1}^{\infty} \frac{N}{N !} \int e^{-W_{N}} \prod^{N-1} \varrho e^{-V} d^{N-1} x
$$

is satisfied pointwise on $\Lambda$. Note that the $V$ so defined is in $L^{\infty}(d x)$.

Lemma 8.15. Suppose $W_{1}=0$ and $W_{N} \geqq-B N$ for each $N$. Then the map $P: L^{1}(d x) \times L^{\infty}(d x) \rightarrow L^{\infty}(d x)$, defined by

$$
P(\varrho, V)=e^{-V(x)}[\Xi(V)]^{-1} \sum_{N=1} \frac{N}{N !} \int e^{-W_{N}} \prod^{N-1} \varrho e^{-V} d^{N-1} x,
$$

satisfies:

(a) $P(\varrho, V)$ is (norm) continuous in $\varrho$ and $V$.

(b) $\left.\frac{\delta P}{\delta V}\right|_{\varrho, V}$ exists and is (norm) continuous in $\varrho$ and $V$.

(c) $\left.\frac{\delta P}{\delta \varrho}\right|_{\varrho, V}$ exists and is (norm) continuous in $\varrho$ and $V$.

(d) $\left[\frac{\delta P}{\delta V}\right]_{\varrho_{0}, V_{0}}^{-1}$ exists for any pair $\left(\varrho_{0}, V_{0}\right)$ satisfying $\varrho_{0}=\varrho_{V_{0}}$.

Proof. This proof parallels that of Lemma 4.2. Again, it is straightforward to verify (a)-(c). In order to prove (d), we evaluate $\frac{\delta P}{\delta V}$ at $\left(\varrho_{0}, V_{0}\right)$ :

$$
-\left.\frac{\delta P}{\delta V}\right|_{\varrho_{0}, V_{0}}(h ; y)=h(y)-\int h(x) d \mu(x)+\int C(x, y) h(x) d \mu(x) .
$$

In (8.63), $d \mu(x)=\varrho_{0}(x) d x$ and

$$
\begin{aligned}
C(x, y)= & \exp \left(-V_{0}(x)\right) \exp \left(-V_{0}(y)\right)[\Xi(V)]^{-1} \\
& \cdot \sum_{N=1}^{\infty} \frac{N(N-1)}{N !} \int e^{-W_{N}} \prod_{i=1}^{N-2} \varrho_{0}\left(x_{i}\right) \exp \left(-V_{0}\left(x_{i}\right)\right) d^{N-2} x .
\end{aligned}
$$

The assumption $W_{N} \geqq-B N$ implies $C(x, y) \in L^{\infty}(d x d y)$.

By reasoning entirely analogous to the proof of Lemma 4.2, it suffices to show that the equation $-\left.\frac{\delta P}{\delta V}\right|_{\varrho_{0}, V_{0}}(h ; y)=0$, for $h \in L^{2}(d \mu)$, possesses only the trivial solution. To this end, suppose that the above equation is satisfied for some $h \in L^{2}(d \mu)$. Then

$$
0=\int h^{2}(y) d \mu(y)-\left[\int h(y) d \mu(y)\right]^{2}+\int C(x, y) h(x) h(y) d \mu(x) d \mu(y),
$$


which may be rewritten in the form

$$
\begin{aligned}
0= & {[\Xi(V)]^{-1} \sum_{N=1}^{\infty}\left[\frac{1}{N !} \int e^{-W_{N}} \prod_{i=1}^{N} \varrho_{0}\left(x_{i}\right) \exp \left(-V_{0}\left(\dot{x}_{i}\right)\right)\right] } \\
& \cdot\left(\sum_{j=1}^{N}\left[h\left(x_{j}\right)-\frac{1}{N} \int h d \mu\right]\right)^{2} d^{N} x .
\end{aligned}
$$

Recalling that $W_{1}=0,(8.66)$ implies

$$
\int d x \varrho_{0}(x) \exp \left(-V_{0}(x)\right)\left[h(x)-\int h d \mu\right]^{2}=0,
$$

and thus $h$ is a constant. However, for a constant $h$, Eq. (8.65) becomes

$$
h^{2}\left(\left\langle N^{2}\right\rangle_{0}-\langle N\rangle_{0}^{2}\right)=0 \text {, }
$$

where \langle\rangle$_{0}$ denotes the expectation value in the state with external potential $U_{0}=-\log \varrho_{0}+V_{0}$. Hence $h \equiv 0$.

The remainder of the proof is identical to that of Lemma 4.2.

Corollary. Suppose $W_{1}=0$ and $W_{N} \geqq-B N$ for each $N$. Then if there exists a $V_{0}$ satisfying $\varrho_{0}=\varrho_{V_{0}}$ :

(1) The inverse map $V(\varrho)$ exists and is continuous in some neighborhood of $\varrho_{0}$.

(2) The inverse map $V(\varrho)$ possesses a Fréchet derivative at $\varrho_{0}$.

Proof. This follows immediately from (a)-(d) and the implicit function theorem.

Remarks. (1) If the $W_{N}$ satisfy the above conditions, and if, in addition, condition (b) of subsection (ii) is satisfied (i.e., if there is some $\tilde{N}>n$ such that $\left.\int W_{\tilde{N}} \prod^{\tilde{N}} \varrho d^{\tilde{N}} x<\infty\right)$, then we already know by the results of subsections (ii) and (iii) that the inverse map exists and is continuous. In this case, the content of the corollary is that the map possesses a Fréchet derivative.

(2) As remarked previously, systems with hard core interactions fail to satisfy condition (b), but do satisfy the above conditions. In this case, the lemma amounts to a proof of local existence of an inverse map (and, moreover, to a proof that it is continuous and differentiable). This means that if we can find a pair $\left(\varrho_{0}, V_{0}\right)$ satisfying $\varrho_{0}=\varrho_{V_{0}}$, then the inverse map exists for densities sufficiently close to $\varrho_{0}$. Of course, it is always easy to find such pairs $\left(\varrho_{0}, V_{0}\right)$, since one can simply compute the density $\varrho(U ; x)$ for some external potential $U$, and then define $V$ by $e^{-U}=\varrho e^{-V}$.

(v) The Inverse Problem for Several Species. In this subsection, we will extend the results of 8 (ii)-8(iv) to systems with several species of particles. It will be shown that, within the formalism of the grand canonical ensemble, the multispecies inverse problem is in fact equivalent to that for a single species.

Let us first define the problem for $K$ species. We denote by $\left\langle\Lambda_{1}, d x_{1}\right\rangle, \ldots,\left\langle\Lambda_{K}, d x_{K}\right\rangle$ the single particle measure spaces of the $K$ individual species. For the purposes of this section, we shall regard the $K$ measure spaces as distinct (i.e., disjoint) even if they are identifiable as the same space.

Let $\mathbf{N}$ be a sequence of $K$ nonnegative integers, $\mathbf{N}=\left(N_{1}, \ldots, N_{K}\right)$. Consider the measure space $\left\langle\Lambda^{\mathbf{N}}, d^{\mathbf{N}} x\right\rangle$, where $\Lambda^{\mathbf{N}}$ denotes the cartesian product, 
$\Lambda^{\mathbf{N}}=\bigotimes_{j=1}^{N_{1}} \Lambda_{1} \ldots \bigvee_{j=1}^{N_{K}} \Lambda_{K}$, and $d^{\mathbf{N}} x=d^{N_{1}} x_{1} \ldots d^{N_{K}} x_{K}$ is the associated product measure. A system of $K$ species is specified by a sequence of measurable functions $\left(W_{\mathbf{N}}: \Lambda^{\mathbf{N}} \rightarrow \mathbb{R}\right)$, one for each $\mathbf{N}=\left(N_{1}, \ldots, N_{K}\right)$.

As in Sect. 5, an external potential is really a collection of $K$ external potentials, $\mathbf{U}=\left(U_{I}: \Lambda_{I} \rightarrow \mathbb{R} \mid I=1, \ldots, K\right)$. The grand canonical partition function in the presence of the external potential $\mathbf{U}$ is given by

$$
\Xi_{\mathbf{U}}=\sum_{\mathbf{N}} \frac{1}{(\mathbf{N}) !} Z_{\mathbf{N}}(\mathbf{U})
$$

where

$$
Z_{\mathbf{N}}(\mathbf{U})=\int_{\Lambda^{\mathbf{N}}} \exp \left(-W_{\mathbf{N}}\right) \prod_{I=1}^{K} \prod_{I_{j}=1}^{N_{I}} \exp \left(-U_{I}\left(x_{I_{J}}\right)\right) d^{\mathbf{N}} x,
$$

and $(\mathbf{N}) !=N_{1} ! \ldots N_{K} !$.

The densities $\varrho_{I}(\mathbf{U} ; x)$ for the $K$ particle species are given by the usual expressions.

In this context, the inverse problem is the following. Given a family of $K$ nonnegative $L^{1}$ functions $\left(\varrho_{I}: \Lambda_{I} \rightarrow \mathbb{R}^{+} \mid I=1, \ldots, K\right)$, does there exist a family of external potentials $\mathbf{U}$ such that $\varrho_{I}=\varrho_{I}(\mathbf{U})$ a.e. $\left[d x_{I}\right]$ for every $I$ ?

We shall show that the above problem is indeed equivalent to a single species inverse problem. Let $\Lambda$ denote the direct sum of the $K$ single particle spaces:

$$
\Lambda=\bigoplus_{I=1}^{K} \Lambda_{I}
$$

(We again emphasize the $K$ spaces are to be regarded as distinct.) For $x \in A$, we may define the $K$ projections

$$
\delta_{I}(x)=\left\{\begin{array}{ll}
1 & x \in \Lambda_{I} \\
0 & \text { otherwise }
\end{array},\right.
$$

so that $\sum_{I} \delta_{I}(x)=1$. Then $\langle\Lambda, d x\rangle$ with $d x \equiv \sum_{I=1}^{K} \delta_{I}(x) d x_{I}$ is of the form of a single particle measure space.

The families of potentials and densities, $\left(U_{I}\right)$ and $\left(\varrho_{I}\right)$, may be identified with the single particle quantities

$$
U(x)=\sum_{I=1}^{K} \delta_{I}(x) U_{I}(x)
$$

and

$$
\varrho(x)=\sum_{I=1}^{K} \delta_{I}(x) \varrho_{I}(x)
$$

Finally, consider all sequences $\mathbf{N}=\left(N_{1}, \ldots, N_{K}\right)$ with $|\mathbf{N}| \equiv \sum_{I=1}^{K} N_{I}=N$. We may construct a single $N$-particle interaction $W_{N}$ from all $\mathbf{N}$-particle interactions $W_{N}$ with $|\mathbf{N}|=N$ according to

$$
W_{N}\left(x_{1}, \ldots, x_{N}\right)=\sum_{|\mathbf{N}|=N_{N}} C_{\mathbf{N}} \delta_{\mathbf{N}}\left(x_{1}, \ldots, x_{N}\right) W_{\mathbf{N}}\left(x_{1_{1}}, \ldots, x_{1_{N_{1}}}, \ldots, x_{K_{1}}, \ldots, x_{K_{N_{K}}}\right),
$$


where the $C_{\mathbf{N}}$ are combinatoric factors and

$$
\delta_{\mathbf{N}}\left(x_{1}, \ldots, x_{N}\right)=\prod_{I=1}^{K} \prod_{I_{J}=1}^{N_{I}} \delta_{I}\left(x_{I_{j}}\right)+\text { permutations } .
$$

In terms of the above quantities, the multispecies grand canonical partition function may be written exactly as in Eq. (8.1). Thus this problem is equivalent to the single species case, and all results of subsections 8(ii)-8(iv) are applicable.

Remarks. (1) The inverse problem for several species can also be solved by generalizing the theorems of subsection (ii) (as was done in Sect. 5 for the canonical ensemble). It is not difficult to show that such a generalization can be proved under condition (A) of subsection (ii) and a set of integral conditions on some of the interactions $W_{\mathbf{N}}$. As an example, it suffices that for each $I=1, \ldots, K$, there is an $N_{I}>n=\sum_{I} \int \varrho_{I} d x_{I}$ such that

$$
\int\left(W_{\mathbf{N}}\right)_{+} \prod^{N_{I}} \varrho_{I} d^{N_{I}} x_{I}<\infty \text { for } \mathbf{N}=\left(0, \ldots, 0, N_{I}, 0, \ldots, 0\right) .
$$

The above method is in fact more general than the mapping described here. For this mapping, condition (b) of subsection (ii) amounts to integral conditions on $\left(\begin{array}{c}N+K-1 \\ K-1\end{array}\right)>K$ of the interactions $W_{\mathbf{N}}$.

(2) The equivalence established here may also be applied in the reverse sense. One can simply express the space $\Lambda$ as a union of disjoint subsets, thereby mapping a single species problem into an equivalent multispecies problem. This may in fact be useful for the analysis of some systems for which the methods of subsection (ii) are inapplicable.

(vi) Systems with Charged Coupling. In this subsection, we investigate the grand canonical inverse problem for a system of charged particles; our main result is Theorem 8.17. The system is characterized by $K>1$ distinct nonzero charges $\left(q_{1}, \ldots, q_{k}\right)$. If we regard particles of the same charge as members of a given species, the problem is readily formulated in the notation of subsection (v). For a given set of charges, the system is specified by a sequence of interactions $\left(W_{\mathbf{N}}: \Lambda^{|\mathbf{N}|} \rightarrow \mathbb{R}\right)$, where $\mathbf{N}=\left(N_{1}, \ldots, N_{K}\right),|\mathbf{N}|=\sum_{I=1}^{K} N_{I}(\mathbf{N})$ and $N_{I}(\mathbf{N})=0,1, \ldots$ is the number of particles of charge $q_{I}$ in the $\mathbf{N}$-particle state. The $W_{\mathbf{N}}$ need not have any symmetry properties.

The grand canonical partition function at fugacity $y$ in the presence of an external potential $U$ is given by

$$
\Xi(U ; y)=\sum_{\mathbf{N}} y^{|\mathbf{N}|} Z_{\mathbf{N}}(U) /(\mathbf{N}) !,
$$

with

$$
Z_{\mathbf{N}}(U)=\int \exp \left(-W_{\mathbf{N}}\right) \prod_{I=1}^{K} \prod_{I_{J}=1}^{N_{I}(\mathbf{N})} \exp \left(-q_{I} U\left(x_{I_{j}}\right)\right) d^{\mathbf{N}} x .
$$

As before, we use the convention $Z_{0}=1$. 
The density of the $I_{j}^{\text {th }}$ particle in the state $\mathbf{N}$, as well as the overall density $\varrho_{I}(U, y)(x)$ of particles of charge $q_{I}$, may be computed in accord with expressions given in Sects. 6 and 8 (ii).

We shall investigate the inverse problem for the charge density $C=\sum_{I} q_{I} \varrho_{I}$, rather than for the particle density $\varrho=\sum_{I} \varrho_{I}$ (see discussion in Sect. 6).

For a single particle species the fugacity, as introduced in Eq. (8.15), is merely a bookkeeping device for setting the zero of the potential. For charged systems the fugacity takes on independent significance (shifting the potential by a constant does not amount to a change in the fugacity). In physical systems, it is associated with the "ambient pressure in some external medium surrounding $\Lambda$."

The ability to adjust this external pressure implies some additional control over the system. In particular, it is expected that one can achieve a specified average number of particles which occupy the system in addition to producing a given charge density.

Indeed, one can further generalize the above problem. Suppose it is possible to adjust, independently, the different fugacities of the different charged species. (This amounts to control over the various partial pressures at infinity.) Then, in principle, it should be possible to specify the expected particle numbers of the individual constituents which together produce a given charge density. This new problem and its variants will be considered briefly at the end of the section.

The relevant functional for the former version of the inverse problem is

$$
\mathfrak{b}_{C, n}(U ; y)=y^{n} \exp \left(-\int C U\right) / \Xi(U ; y),
$$

where $n<\infty$ is the desired particle number and $C \in L^{1}(d x)$, the charge density.

The assumptions under which we prove the existence of an inverse map are the natural extensions of the conditions imposed in Sects. 6 and 8(ii). In the canonical ensemble, we introduced the notion of a $\gamma$-decomposition which permitted us to define meaningful (positive) measures $d^{N} \mu_{\gamma}$. It was then required that the positive part of the interaction be summable with respect to some measure of the above type. A similar decomposition will be defined for the grand canonical ensemble. Here, however, we will only require that a few of the $\left(W_{\mathbf{N}}\right)_{+}$be summable with respect to the appropriate measures, so that most of the $W_{\mathbf{N}}$ may be badly behaved (e.g., hard core).

For the existence theorem of subsection 8 (ii), it was required that there exist some admissible potential (which by shifting was taken to be zero) such that the corresponding partition function is finite at finite fugacity. Similar considerations dictate that we impose such a condition here.

Definition. Let $C \in L^{1}(d x)$ and let $n>0$ be a finite number. Without loss of generality, we assume $\int C_{+} \geqq \int C_{-}$.

Let $n_{1}, \ldots, n_{K}$ be a set of nonnegative constants which satisfy $\sum_{I} n_{I}=n, \sum_{I} n_{I} q_{I}$

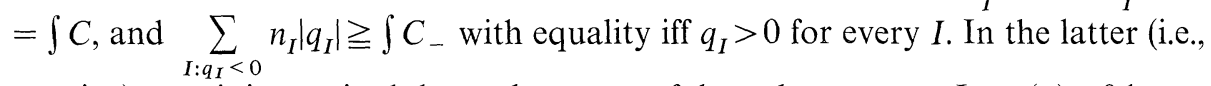
massive) case, it is required that at least two of the $n_{I}$ be nonzero. Let $\gamma(x)>0$ be an $L^{1}$ function of unit norm. 
The functions $\alpha(x)=C_{+}(x)+\Delta \gamma(x)$ and $\beta(x)=C_{-}(x)+\Delta \gamma(x)$, with $\Delta>0$ chosen so that $\int \alpha=\sum_{I: q_{I}>0} n_{I} q_{I}$ (and hence $\left.\int \beta=\sum_{I: q_{I}<0} n_{I}\left|q_{I}\right|\right)$, are referred to as the $\gamma$-decomposition of the charge density $C$ at particle number $n$.

Remark. For the grand canonical ensemble, it is seen that we decompose the desired particle number as well as the given charge density. Note that the existence of numbers $n_{I}$ satisfying the above criteria is not a priori guaranteed. In fact, it is not difficult to show that this is a necessary condition.

For the massive case $\left(q_{I}>0\right.$ for every $\left.I\right)$, this requirement prevents total domination by a single species. Densities of this form cannot be realized, except in some limiting sense.

For the charged case, the conditions on the $n_{I}$ preclude total charge separation, which is also only achievable in a limiting sense unless every $W_{\mathbf{N}}$ forces charge polarization. This latter situation may be regarded as a peculiar and rather unphysical case of hard core interactions. Systems similar to this are briefly discussed in Sect. 9.

We consider pairs $(U, y)$ in the set

$$
\mathscr{U}_{\gamma}=\left\{\left(U: \Lambda \rightarrow \mathbb{R}, y \in \mathbb{R}^{+}\right) \mid U \in L^{1}\left(d \mu_{\alpha}\right) \cap L^{1}\left(d \mu_{\beta}\right), \Xi(U ; y)<\infty\right\} .
$$

As before, $d \mu_{\alpha}=\alpha d x / \int \alpha$ and $d \mu_{\beta}=\beta d x / \int \beta$. We shall often denote either $d \mu_{\alpha}$ or $d \mu_{\beta}$ simply by $d \mu_{\gamma}$; the intended measure should be clear from the context. We shall also use the notation $d^{\mathbf{N}} \mu_{\gamma}$ in analogy with Eq. (6.17).

Proposition 8.15. Suppose there exists a $\gamma$-decomposition of $(C, n)$ satisfying $\alpha \log \alpha \in L^{1}(d x), \beta \log \beta \in L^{1}(d x)$ and such that for each $I$ with $n_{I}>0$ in the $\gamma$-decomposition, there is an $\tilde{N}_{I}>n$ so that for $\mathbf{N}=\left(0, \ldots, \tilde{N}_{I}, \ldots, 0\right),\left(W_{\mathbf{N}}\right)_{+} \in L^{1}\left(d^{\mathbf{N}} \mu_{\gamma}\right)$. Then $\mathfrak{b}_{C, n}(U ; y)$ is uniformly bounded above in $\mathscr{U}_{\gamma}$.

Remark. It will become clear from the proof of this proposition and the subsequent theorem that there are many other choices of $\mathbf{N}$ such that summability of $\left(W_{\mathbf{N}}\right)_{+}$with respect to some $\gamma$-decomposition provides the necessary stability. The above is only an example, perhaps the simplest, of many possible sufficient conditions.

Proof. For $U \in \mathscr{U}_{\gamma}$, we use the identity

$$
y^{n} \exp \left(-\int C U\right)=\prod_{I=1}^{K}\left[y \exp \left(-q_{I} \int U d \mu_{\gamma}\right)\right]^{n_{I}} .
$$

Then

$$
\mathfrak{G}_{C, n}(U ; y) \leqq \prod_{I=1}^{K}\left[y \exp \left(-q_{I} \int U d \mu_{\gamma}\right)\right]^{n_{I}} /\left\{1+\sum_{I=1}^{K} D_{I}\left[y \exp \left(-q_{I} \int U d \mu_{\gamma}\right)\right]^{\tilde{N}_{I}}\right\},
$$

where the coefficients $D_{I}$ have been obtained by using Jensen's inequality for the $\tilde{W}_{\mathrm{N}}$, as in Eq. (6.21). Thus

$$
\mathfrak{b}_{C, n}(U ; y) \leqq \max _{I}\left\{1,1 / D_{I}\right\} .
$$

Theorem 8.16. Under the hypotheses of Proposition 8.15 and the additional assumption that $\mathscr{U}_{\gamma} \neq \emptyset$, there is a unique $(U, y) \in \mathscr{U}_{\gamma}$ which maximizes $\mathfrak{b}_{C, n}$ in $\mathscr{U}_{\gamma}$. 
Proof. Let $\left(U_{m}, y_{m}\right)$ be a maximizing sequence for $\mathfrak{G}_{C, n}$ in $\mathscr{U}_{\gamma}$. By an argument similar to that in Propositions 8.2 and 8.3, both the numerator and denominator of $\tilde{\mathfrak{b}}_{C, n}$ are eventually bounded above and below. In fact a stronger statement can be made. For every $I, y_{m} \exp \left(-q_{I} \int U_{m} d \mu_{\gamma}\right)$ is bounded away from zero and infinity.

The functions $\pi\left[y_{m}^{1 / 2} \exp \left(-Q \cdot U_{m} / 2\right)\right]$ may be regarded as elements of the space $\mathbb{L}^{2}(d \sigma)$, which is the direct sum of the spaces $L^{2}\left(d \sigma_{\mathbf{N}}\right), d \sigma_{\mathbf{N}}=e^{-W_{\mathbf{N}}} d^{\mathbf{N}} x$, defined as in Eq. (8.11). Since by the above argument $\Xi\left(U_{m} ; y_{m}\right)=\| \pi\left[y_{m}^{1 / 2} \exp \left(-Q \cdot U_{m} / 2\right) \|_{2(d \sigma)}^{2}\right.$ is bounded, some subsequence converges weakly in $\mathbb{L}^{2}(d \sigma)$. It is not difficult to extend the reasoning of Theorem 6.2 to show that this subsequence converges strongly in $\mathbb{L}^{2}(d \sigma)$.

We claim that the strong limit is a pair $(U, y) \in \mathscr{U}_{\gamma}$. First, let us show that the fugacities $y_{m}$ are bounded away from zero and infinity. Observe that the hypotheses imply there are at least two (single charge species) states, which we shall call $\mathbf{L}$ and $\mathbf{M}$, such that the measures $d \sigma_{\mathbf{L}}$ and $d \sigma_{\mathbf{M}}$ are equivalent to the underlying product measures. Furthermore, the functions $\prod_{i=1}^{L} y_{m}^{1 / 2}$ $\cdot \exp \left(-q_{L} U_{m}\left(x_{i}\right) / 2\right)$ and $\prod_{i=1}^{M} y_{m}^{1 / 2} \exp \left(-q_{M} U_{m}\left(x_{i}\right) / 2\right)$ have strong, and hence pointwise, limits with respect to the measures $d \sigma_{\mathbf{L}}$ and $d \sigma_{\mathbf{M}}, q_{L} \neq q_{M}$. Let us denote these limits by $F_{L}\left(x_{1}, \ldots, x_{L}\right), F_{M}\left(x_{1}, \ldots, x_{M}\right)<\infty$ a.e., and let $d^{L} \sigma_{\mathbf{M}}$ denote the measure $\bigotimes_{p=1}^{L} d \sigma_{\mathbf{M}}$ on $\Lambda^{L M}$, and similarly for $d^{M} \sigma_{\mathbf{L}}$. Using an argument along the lines of Theorem 6.5 and the fact that each term in the numerator of $\mathbf{5}_{C, n}$ is individually bounded above and below, it can be shown that $F_{L}$ and $F_{M}$ are nonzero a.e. Now

$$
\prod_{p=1}^{L} \prod_{i=1}^{M} y_{m}^{1 / 2} \exp \left(-q_{M} U_{m}\left(x_{i}^{p}\right) / 2\right) \rightarrow \prod_{p=1}^{L} F_{M}\left(x_{1}^{p}, \ldots, x_{M}^{p}\right)
$$

pointwise a.e. However, this limit also equals

$$
\lim _{m \rightarrow \infty}\left[\left(y_{m}^{M L / 2}\right)^{\left(q_{L} / q_{M}\right)-1}\right] \prod_{p=1}^{M} F_{L}\left(x_{1}^{p}, \ldots, x_{L}^{p}\right) .
$$

Thus, at least for a further subsequence, the limit of the $y_{m}$ exists and is not zero or infinite. Then, it is not difficult to see that the $U_{m}(x)$ converge pointwise to some function $U$ such that $\Xi(U ; y)<\infty$.

The inequality $\lim _{m \rightarrow \infty} y_{m}^{n} \exp \left(-\int C U_{m}\right) \leqq y^{n} \exp \left(-\int C U\right)$ is easily established by methods used previously. This implies $(U, y) \in \mathscr{U}_{\gamma}$ and $\mathfrak{6}_{C, n}(U ; y)$ $=\sup _{(U, y) \in \mathscr{U}} \mathfrak{G}_{C, n}(U ; y)$. Uniqueness ${ }^{2}$ is established using Cauchy-Schwarz as in Proposition 6.4.

Theorem 8.17. Under the hypotheses of Theorem 8.16 and the additional assumption that there is a $U_{0} \in \mathscr{U}_{\gamma}$ (which, by shifting, can be set to zero) such that $\Xi\left(U_{0} ; y\right)<\infty$ for all finite $y, C_{(U, y)}(x)=C(x)$ a.e. and $\langle N\rangle_{(U, y)}=n$.

2 There is one exception to the uniqueness, and that is when $W_{N} \equiv \infty$ for all nonneutral states. In this case, we have uniqueness up to a constant. (Although such a system violates the hypotheses of Theorem 8.16, it is not difficult to provide similar conditions under which the theorem holds for this case.) Such systems do in fact occur in the semiclassical approximation to certain nonabelian quantum field theories 
Proof. First we establish that the expected particle number in the maximizing state is finite (and thus that the charge density is well-defined). We note that this is independent of the additional hypothesis. For any pair $(U, y)$ such that $\Xi(U ; y)<\infty, \Xi\left(U ; y^{\prime}\right)$ is analytic for all $y^{\prime}<y$. Thus by lowering the fugacities of a maximizing sequence $\left(U_{m}, y_{m}\right)$ by an amount tending to zero, we may with no loss of generality assume that, for each $m, \Xi\left(U_{m}, y\right)$ is analytic provided that $\left|y_{m}-y\right|$ is sufficiently small. Now if the partition function is analytic about some fugacity $y_{m}$, the particle number surely exists. Suppose $\langle N\rangle_{\left(U_{m}, y_{m}\right)}>n$. Since $y \partial / \partial y[\mathfrak{G}(U ; y)]$ $=\mathfrak{G}(U ; y)\left[n-\langle N\rangle_{(U, y)}\right]$, it is clear that by lowering the fugacity further, we obtain an improved maximizing sequence which satisfies $\langle N\rangle_{\left(U_{m}, y_{m}\right)} \leqq n$. Recalling the strong convergence, $\Xi\left(U_{m}, y_{m}\right) \rightarrow \Xi(U ; y)$, it is seen, by Fatou, that the above inequality persists in the limit (i.e., $\langle N\rangle_{(U, y)} \leqq n$ ).

Thus the positive and negative particle densities, $\varrho_{+}(U, y) \equiv \sum_{I: q_{I}>0} \varrho_{I}(U, y)$ and $\varrho_{-}(U, y)$, are separately well-defined. (These are not, of course, the positive and negative parts of $C_{(U, y)}$.

Next consider the pair $((1-\varepsilon) U, y)$ with $\varepsilon>0$. It is clear that $((1-\varepsilon) U, y) \in \mathscr{U}_{\gamma}$ since

$$
\Xi((1-\varepsilon) U ; y) \leqq \Xi(U ; y)^{1-\varepsilon} \Xi(0 ; y)^{\varepsilon}
$$

by Hölder's inequality. In fact, (8.84) also establishes that $\lim _{\varepsilon \rightarrow 0} \Xi((1-\varepsilon) U ; y)$ $=\Xi(U ; y)$ since, by Fatou, $\lim _{\varepsilon \rightarrow 0} \Xi((1-\varepsilon) U ; y) \geqq \Xi(U ; y)$.

By similar reasoning, we will show that $\varrho_{ \pm}((1-\varepsilon) U, y) \rightarrow \varrho_{ \pm}(U, y)$ weakly in $L^{1}(d x)$. First observe that

$$
\varrho_{ \pm}((1-\varepsilon) U, y)(x) \leqq \frac{\Xi(U ; y)^{1-\varepsilon} \Xi(0 ; y)^{\varepsilon}}{\Xi((1-\varepsilon) U ; y)} \varrho_{ \pm}(U, y)^{1-\varepsilon}(x) \varrho_{ \pm}(0, y)^{\varepsilon}(x) .
$$

Weak convergence is established by integrating (8.85) over any set of positive measure and using the Hölder inequality.

It is now straightforward to show that $C_{(U, y)}=C$ a.e. and that $\langle N\rangle_{(U, y)}=n$. Let

$$
h(x)=\left\{\begin{array}{ll}
+1 & C_{(U, y)}(x) \geqq C(x) \\
-1 & C_{(U, y)}(x)<C(x)
\end{array},\right.
$$

and consider the trial function $T_{\varepsilon}=\left(1-\varepsilon^{2}\right) U+\varepsilon h$. As before, $T_{\varepsilon} \in \mathscr{U}_{\gamma}$. Now $\mathfrak{5}\left(T_{\varepsilon} ; y\right)$ $=\mathfrak{G}\left(\left(1-\varepsilon^{2}\right) U ; y\right) \cdot\left[1+\varepsilon \int\left[C_{\left(\left(1-\varepsilon^{2}\right) U, y\right)}-C\right] h+O\left(\varepsilon^{2}\right)\right]$. However $\quad\left(\mathfrak{G}\left(\left(1-\varepsilon^{2}\right) U ; y\right)\right.$ $=\mathfrak{b}(U ; y)+O\left(\varepsilon^{2}\right)$, so that

$$
\mathfrak{5}\left(T_{\varepsilon} ; y\right)=\mathfrak{5}(U ; y)\left[1+\int\left|C_{(U, y)}-C\right|\right]+o(\varepsilon),
$$

by the weak $L^{1}$ convergence demonstrated above. Since $(U, y)$ is the maximizer, the integrand in (8.87) is zero a.e. [ $d x]$. In analogous fashion, it is easily shown that $\langle N\rangle_{(U, y)}=n$.

Remarks. 1) In certain systems one can control the partial pressures of the different species and thus it should be possible to produce a specified charge density and a set of expected particle numbers $\left(C, n_{1}, \ldots, n_{K}\right)$. It should be reasonably clear that this problem is solvable by the method of this section, with only minor 
modifications. The relevant functional is

$$
\mathfrak{G}\left(U ; y_{1}, \ldots, y_{K}\right)=\prod_{I=1}^{K} y_{I}^{n_{I}} \exp \left(-\int C U\right) / \Xi\left(U ; y_{1}, \ldots, y_{K}\right),
$$

where

$$
\Xi\left(U ; y_{1}, \ldots, y_{K}\right)=\sum_{\mathbf{N}} \prod_{I} y_{I}^{N_{I}(\mathbf{N})} Z_{\mathbf{N}}(U) /(\mathbf{N}) ! .
$$

Of course, any intermediate case in which only certain fugacities are adjustable is also solvable.

2) Finally, for any of the above problems, we claim that among all the potentials and fugacities for which one can define a charge density, only one pair $(U, \mathbf{y})$ gives the correct charge density and particle number(s). To see this, first note that a proof along the lines of previous uniqueness theorems shows that for a fixed fugacity $\mathbf{y}$, there is at most one potential which produces a given charge density. However, if $C_{\left(U_{1}, \mathbf{y}_{1}\right)}=C_{\left(U_{2}, \mathbf{y}_{2}\right)}$, it can be shown that, for every $I,\left\langle N_{I}\right\rangle_{\left(U_{1}, \mathbf{y}_{1}\right)}$ $>\left\langle N_{I}\right\rangle_{\left(U_{2}, \mathbf{y}_{2}\right)} \Leftrightarrow y_{I}\left(\mathbf{y}_{1}\right)>y_{I}\left(\mathbf{y}_{2}\right)$. This implies the asserted uniqueness.

\section{Hard Core Interactions}

In this section, we briefly discuss systems with hard core interactions and show that these systems are capable of exhibiting counterexamples to the fundamental existence and uniqueness theorems of Sects. 2 and 8.

A set of necessary conditions for existence of an inverse map will be derived in the context of the canonical ensemble. (The generalization of these conditions to the grand canonical ensemble is straightforward and, in practice, may be easier to verify.) Finally, for the grand canonical ensemble, we shall discuss the implications of our partial results [derived in Sect. 8(iv)] on the set of densities for which an inverse map exists.

Definition. Let $\langle\Lambda, d x\rangle$ be a $\sigma$-finite measure space. A measurable function $W_{N}: \Lambda^{N} \rightarrow \mathbb{R} \cup\{\infty\}$ is said to be a hard core interaction if the set $H_{N}=\left\{X \in \Lambda^{N} \mid W_{N}(X)=\infty\right\}$ has a positive $\left[d^{N} x\right]$ measure. A sequence of interaction potentials $\left(W_{N} \mid N=1,2, \ldots\right)$ is said to be hard core if for every $N \geqq 2, W_{N}$ is hard core.

Example. The best known example is the case of hard spheres for which $\Lambda \subset \mathbb{R}^{d}$ and

$$
W_{N}\left(x_{1}, \ldots, x_{N}\right)= \begin{cases}\infty ; & \text { if for some } i \neq j,\left|x_{i}-x_{j}\right|<2 a \\ T_{N}\left(x_{1}, \ldots, x_{N}\right) ; & \text { otherwise }\end{cases}
$$

Here $a$ is the so-called hard core radius, and $T_{N}\left(x_{1}, \ldots, x_{N}\right)$ is the "tail" which satisfies $-\infty<T_{N}<\infty$ a.e. $\left[d^{N} x\right]$.

Remarks. 1) For the grand canonical ensemble, it was demonstrated that if, for a single $N>\int \varrho, W_{N}$ is not hard core, then under quite general assumptions an inverse map exists. However, it is usually the case that if a system has hard core interactions for some $\tilde{N}$, then for all $N>\tilde{N}$ the $W_{N}$ are also hard core. Typically $\tilde{N}=2$; hence the restriction " $W_{N}$ hard core for all $N \geqq 2$ " in the previous definition. 
On the other hand, most systems of physical interest satisfy $Z_{0}=1$ and $e^{-W_{1}}=1$. Under these circumstances, the results of Sect. 8(ii) guarantee that the inverse map exists whenever $\int \varrho<1$. This, however, is only a small deviation from the uninteresting case of a single isolated particle and we do not consider it a meaningful result on hard core systems.

2) We also do not consider interactions for which $\Lambda^{N} \backslash H_{N}$ is a rectangle (i.e., of the form $\left.a_{1} \times a_{2} \times \ldots \times a_{N}, a_{i} \subset A\right)$ to be realistic hard core potentials. For the canonical ensemble, this is simply a generalization of the problem treated in Sect. 5, and can be handled by the methods of this paper, with the following result:

Provided that $\int\left(T_{N}\right)_{+} \prod^{N} \varrho d^{N} x<\infty$ and $Z_{N}(0)<\infty$, the necessary and sufficient condition for the existence of an inverse map is that there exist non-negative functions $\eta_{1}, \ldots, \eta_{N}, \int \eta_{i} d x=1,\left.\eta_{i}\right|_{\Lambda \backslash a_{i}}=0$ with $\sum_{i} \eta_{i}(x)=\varrho(x)$.

The hard core part of interactions of the above form is not an "interacting" hard core; it is simply a restriction on the sets on which the various particles are permitted to live. Henceforth, when we discuss hard core interactions, it is the example of hard spheres that should be kept in mind.

Counterexamples. Most types of counterexamples can be found in the simplest nontrivial hard core system: a one-dimensional gas of (no more than) two particles.

Let $W(x, y)$ be a potential of the form (9.1) on the region $\Lambda^{2}=(0,2 a+b)^{2}$ with $b<a$.

1) Non-Uniqueness (Canonical ensemble). Let $U(x)$ be any potential and let $\varrho(U ; x)$ be the corresponding density. It is easily seen that a pointwise identical density is produced if the applied potential is $U(x)+\alpha \chi_{(0, b)}+\beta \chi_{(2 a, 2 a+b)}$ for any constants $\alpha, \beta \in \mathbb{R}$. An entire family of potentials all produce the same density! This is a consequence of a phenomenon which we call trapping.

2) Non-Existence (Canonical ensemble). Let $U(x)$ be any measurable function which satisfies $0<Z(U)<\infty$. (In other words, $U$ must be a function from which it is possible to compute a density.) It is easy to verify that $\int_{0}^{b} \varrho(U ; x) d x$ $=\int_{2 a}^{2 a+b} \varrho(U ; x) d x=1$. Evidently any function $\varrho$ which does not satisfy the above integral constraints cannot be interpreted as the equilibrium density of this system under the action of any external potential.

3) Non-Existence (Grand canonical ensemble). Here we set $Z_{0}=1$ and $e^{-W_{1}}=1$. Then for any potential $U(x)$ satisfying $0<\Xi(U)<\infty$, we have $\int_{b}^{2 a} \varrho(U ; x)<1$. Densities not satisfying this conditional restriction cannot be achieved.

This example and the previous one illustrate a phenomenon which we call caging.

We now derive necessary conditions for existence (and uniqueness) of an inverse map. We shall supplement all definitions with examples from the hard sphere gas. For notational simplicity, assume that all interactions are symmetric. 
Definitions. 1) Let $x \in \Lambda$. Let $E(x)$ be the set $\left\{y \in \Lambda \mid \exp \left[-W\left(x, y, x_{3}, \ldots, x_{N}\right)\right]=0\right.$ for all $\left.\left(x_{3}, \ldots, x_{N}\right) \in \Lambda^{N-2}\right\}$. We call $E(x)$ the environment of $x$.

Example. For hard spheres $E(x) \supset B_{2 a}^{\Lambda}(x)$, where $B_{2 a}^{\Lambda}(x)$ is the intersection of $\Lambda$ with a ball of radius $2 a$, centered at $x$. If the gas is sufficiently dilute, $B_{2 a}^{\Lambda}(x)=E(x)$, but under extreme packing $E(x)$ contains a great deal more of $\Lambda$.

2) Let $C \subset A$ be a measurable set. $C$ is said to be a cage if $C \subset \bigcap_{x \in C} E(x)$.

Simply stated, if a given particle occupies a cage, then with probability one, all other particles are excluded.

Example. For hard spheres, $B_{a}^{\Lambda}(x)$ is a cage for every $x \in \Lambda$.

3) A set $T \subset \Lambda$ is said to be a trap if

(i) $T$ is a cage, and

(ii) $\exp \left[-W\left(x_{1}, \ldots, x_{N}\right)\right]=0$ unless, for some $i, x_{i} \in T$.

Example. The sets $(0, b)$ and $(2 a, 2 a+b)$ in the counterexample to uniqueness are traps.

Theorem 9.1. Let $W\left(x_{1}, \ldots, x_{N}\right)$ be a hard core potential and let $U(x)$ be any external potential such that $0<Z(U)<\infty$. Let $\varrho(U ; x)$ be the canonical density of the system. Then:

(a) For every cage $A \subset \Lambda$,

$$
\int_{A} \varrho(U ; x) \leqq 1
$$

with equality iff $A$ is a trap.

(b) For every trap $A \subset A$, the potential $U_{\alpha}(x)=U(x)+\alpha \chi_{A}(x), \alpha \in \mathbb{R}$ satisfies $\varrho\left(U_{\alpha} ; x\right)=\varrho(U ; x)$ pointwise.

Proof. These are straightforward computations.

Corollary. For any nonnegative $\varrho \in L^{1}(d x)$ and for every cage $A \subset \Lambda,(9.2)$ is a necessary condition for the inverse problem to possess a solution.

In particular, for a gas of $N>1$ hard spheres, any density $\varrho$ for which an inverse map exists must satisfy

$$
\int_{B_{a}^{A}(x)} \varrho \leqq 1
$$

for every $x$, with equality iff $B_{a}^{A}(x)$ contains a trap. This is also necessary in the case of the grand canonical ensemble (see Remark 2 below).

Remarks. 1) There are higher order generalizations of all the above notions (e.g. 2environments, 2-cages, etc.). These are nontrivial if the hard core part of the interaction does not obey two-body decomposition.

2) The extension of the above ideas to the grand canonical ensemble is straightforward. Simply define the relevant set for each $N$ and take their intersection. For example, the environment of a point $x$ is given by $E(x)$ $=\bigcap_{N=1}^{\infty} E_{N}(x)$. By convention, the set $C_{1}=\left\{x \in \Lambda \mid e^{-W_{1}}>0\right\}$ is an environment of every point in the single particle state. 
Generically, there are no traps in grand canonical systems. In addition, there are no constraints which require that any admissible density must vanish on certain measurable sets. (Compare counterexamples 2 and 3.) For these reasons, we believe that the analysis of hard core interactions should be easier in the context of the grand canonical ensemble.

In particular, under some additional hypotheses, it was shown in Sect. 8(iv) that if $\varrho$ is any admissible density, then $\varrho^{\prime}$ is also admissible provided that $\left\|\varrho-\varrho^{\prime}\right\|_{1(d x)}$ is sifficiently small. This implies that the set of admissible densities is open. (It may also be possible to show that this set is convex.) We therefore expect that in quite general circumstances, sufficient conditions are of the form $\int_{A_{\alpha}} \varrho<C_{\alpha}$, where $C_{\alpha} \in \mathbb{R}$ and the $A_{\alpha}$ are some collection of sets determined exclusively by the hard core part of the interactions.

The necessary conditions given in the corollary to Theorem 9.1 are precisely of the above form. In this context, it is instructive to examine the formal solutions by Percus [6,7] and Robledo and Varea [8] of the one-dimensional two-body problem. Their expressions for the inverse function are well-defined if and only if, for every $x, \int_{x}^{x+2 a} \varrho(\xi) d \xi<1$. The implication of these solutions is that (9.3) is also a sufficient condition, at least in one dimension.

\section{Appendix: Convergence of Product Functions}

In this appendix we investigate convergence of product functions on a space with a nonproduct measure. Our principal result (Theorem A.3) shows that if the given measure is equivalent to a product measure, then the weak limit of a sequence of product functions is a product function. Counterexamples are provided which show that the hypothesis of equivalence is not superfluous. The method of proof (due to M. Aizenman and E. Nelson) is to use duplication of variables. We treat the case $N=2$; the generalization is obvious.

Additional results on convergence of product functions are found in later theorems.

Definition. Let $\mu$ and $v$ be two measures on a space $\Lambda$. If $\mu(S)=0 \Rightarrow v(S)=0$ for $S \subset \Lambda$, then $v$ is said to be absolutely continuous with respect to $\mu$, denoted by $v \ll \mu$. If $v \ll \mu$ and $\mu \ll v, \mu$ and $v$ are said to be equivalent, denoted by $\mu \sim v$.

Let $\left\langle\Lambda_{x}, \mu_{x}\right\rangle$ and $\left\langle\Lambda_{y}, \mu_{y}\right\rangle$ be $\sigma$-finite measure spaces, and let $\left\langle\Lambda^{2}, \mu_{x y}\right\rangle$ denote their cartesian product. We shall denote by $v_{x y}$ another measure on $\Lambda^{2}$ with $v_{x y} \sim \mu_{x y}$. Finally, we denote by $\Lambda^{4}$ the direct product of two copies of $\Lambda^{2}$.

Definition. A $\mu_{x y}$-measurable function $F: \Lambda^{2} \rightarrow \mathbb{R}$ is said to be a product function if there exist $\mu_{x^{-}}$and $\mu_{y}$-measurable functions, $f: \Lambda_{x} \rightarrow \mathbb{R}$ and $g: \Lambda_{y} \rightarrow \mathbb{R}$, such that $F(x, y)=f(x) g(y)$ a.e. $\left[\mu_{x y}\right]$.

Proposition A.1. If $H: \Lambda^{4} \rightarrow \mathbb{R}$ is $v_{x_{1} y_{1}} v_{x_{2} y_{2}}$-measurable, then $H^{*}: \Lambda^{4} \rightarrow \mathbb{R}$ defined by

$$
H^{*}\left(x_{1}, y_{1}, x_{2}, y_{2}\right)=H\left(x_{1}, y_{2}, x_{2}, y_{1}\right)
$$

is also $v_{x_{1} y_{1}} v_{x_{2} y_{2}}$-measurable. 
Proof. First, we claim

$$
v_{x_{1} y_{1}} v_{x_{2} y_{2}} \sim v_{x_{1} y_{2}} v_{x_{2} y_{1}}
$$

Clearly $v_{x_{1} y_{1}} v_{x_{2} y_{2}} \sim \mu_{x_{1} y_{1}} \mu_{x_{2} y_{2}}$ and $v_{x_{1} y_{2}} v_{x_{2} y_{1}} \sim \mu_{x_{1} y_{2}} \mu_{x_{2} y_{1}}$. Thus it suffices to demonstrate that $\mu_{x_{1} y_{1}} \mu_{x_{2} y_{2}} \sim \mu_{x_{1} y_{2}} \mu_{x_{2} y_{1}}$. Indeed, the latter two measures are identical. To see this, let $T_{x}$ and $T_{y}$ denote the $\sigma$-algebras of $\left\langle\Lambda_{x}, \mu_{x}\right\rangle$ and $\left\langle\Lambda_{y}, \mu_{y}\right\rangle$, respectively. The ( $\sigma$-finite) product measure $\mu_{x y}$ is determined uniquely by the condition that for any measurable rectangle, $R_{x} \times R_{y}$, with $R_{x} \in T_{x}$ and $R_{y} \in T_{y}, \mu_{x y}\left(R_{x} \times R_{y}\right)$ $=\mu_{x}\left(R_{x}\right) \mu_{y}\left(R_{y}\right)$. Noting the analogous conditions which determine the product measures $\mu_{x_{1} y_{1}} \mu_{x_{2} y_{2}}$ and $\mu_{x_{1} y_{2}} \mu_{x_{2} y_{1}}$, it follows from uniqueness that the measures are identical.

Next we note that if the set $S \subset \Lambda^{4}$ is $v_{x_{1} y_{1}} v_{x_{2} y_{2}}$-measurable, then $S^{*}=\left\{\left(x_{1}, y_{1}, x_{2}, y_{2}\right) \in \Lambda^{4} \mid\left(x_{1}, y_{2}, x_{2}, y_{1}\right) \in S\right\} \quad$ is $v_{x_{1} y_{2}} v_{x_{2} y_{1}}$-measurable and hence by Eq. (A-2), $S^{*}$ is $v_{x_{1} y_{1}} v_{x_{2} y_{2}}$-measurable. In fact, $v_{x_{1} y_{1}} v_{x_{2} y_{2}}\left(S^{*}\right)=v_{x_{1} y_{2}} v_{x_{2} y_{1}}(S)$.

Let $H: \Lambda^{4} \rightarrow \mathbb{R}$ be $v_{x_{1} y_{1}} v_{x_{2} y_{2}}$-measurable. Then by the above reasoning, for every $a, b \in \mathbb{R},\left(H^{-1}(a, b)\right)^{*}$ is $v_{x_{1} y_{1}} v_{x_{2} y_{2}}$-measurable. However, $\left(H^{-1}(a, b)\right)^{*}=\left(H^{*}\right)^{-1}(a, b)$, and hence the latter set is also $v_{x_{1} y_{1}} v_{x_{2} y_{2}}$-measurable.

Remark. The switching transformation employed in Proposition A.1 is only welldefined if $v_{x y} \sim \mu_{x y}$. In particular, if the measures are not equivalent, then the sets $S^{*}$, and hence the functions $H^{*}$, are in general not measurable.

Proposition A.2. Let $F: \Lambda^{2} \rightarrow \mathbb{R}, F \in L^{p}\left(v_{x y}\right)$ with $1 \leqq p<\infty$. Then $F$ is a product function iff the function $H: \Lambda^{4} \rightarrow \mathbb{R}$ defined by $H\left(x_{1}, y_{1}, x_{2}, y_{2}\right)=\left|F\left(x_{1}, y_{1}\right) F\left(x_{2}, y_{2}\right)\right|$ satisfies $H=H^{*}$ a.e.

Proof. Here we only consider the case $F \in L^{1}\left(v_{x y}\right)$ and $F \geqq 0$ a.e.; to prove the other cases, simply replace $F$ by $|F|^{p}$ where appropriate.

The condition is clearly necessary.

Suppose the condition is satisfied. Let $g: \Lambda_{y} \rightarrow \mathbb{R}$ be the $\mu_{y}$-measurable function defined by $g(y)=\int F(x, y) v(x, y) d \mu_{x}$, where $v: \Lambda^{2} \rightarrow \mathbb{R}$ is the $\mu_{x y}$-measurable function satisfying $0<v(x, y)<\infty$ a.e. and $v_{x y}(\cdot)=\int(\cdot) v(x, y) d \mu_{x y}$. Clearly, the set $S=\left\{y \in \Lambda_{y} \mid g(y)>0\right\}$ is $\mu_{y}$-measurable.

If $\mu_{y}(S)=0$, then $F \equiv 0$ is certainly a product function. Hence suppose $\mu_{y}(S)>0$. For a.e. $y_{1} \in \Lambda_{y}$,

$$
g\left(y_{1}\right) F\left(x_{2}, y_{2}\right)=F\left(x_{2}, y_{1}\right) \int F\left(x_{1}, y_{2}\right) v\left(x_{1}, x_{2}\right) d \mu_{x_{1}},
$$

for a.e. $x_{2} \in \Lambda_{x}$ and $y_{2} \in \Lambda_{y}$. For fixed $y_{1} \in S$, Eq. (A-3) expresses $F\left(x_{2}, y_{2}\right)$ as a product function of the desired form.

Theorem A.3. Let $\left(F_{n}: \Lambda^{2} \rightarrow \mathbb{R}\right)$ be a sequence of product functions converging weakly in $L^{p}\left(v_{x y}\right), 1 \leqq p<\infty$ :

$$
F_{n} \rightarrow F
$$

Then $F$ is also a product function.

Proof. The sequence of functions $\left(F_{n}\left(x_{1}, y_{1}\right) F_{n}\left(x_{2}, y_{2}\right): \Lambda^{4} \rightarrow \mathbb{R}\right)$ converges weakly in $L^{p}\left(v_{x_{1} y_{1}} v_{x_{2} y_{2}}\right)$ to $F\left(x_{1}, y_{1}\right) F\left(x_{2}, y_{2}\right)$. By Proposition A.2, it suffices to show that the weak limit equals $F\left(x_{1}, y_{2}\right) F\left(x_{2}, y_{1}\right)$ a.e. 
Let $R(p)$ be the set of $L^{p}$ functions which are invariant under the switching transformation [Eq. (A-2)]:

$$
R(p)=\left\{G \in L^{p}\left(v_{x_{1} y_{1}} v_{x_{2} y_{2}}\right) \mid G^{*}=G \text { a.e. }\left[v_{x_{1} y_{1}} v_{x_{2} y_{2}}\right]\right\} .
$$

The desired result is established by showing that $R(p)$ is weakly closed. Since $R(p)$ is linear, and hence convex, this is of course equivalent to showing that $R(p)$ is strongly closed.

Suppose the sequence $\left(G_{n} \in R(p)\right)$ converges $L^{p}\left(v_{x_{1} y_{1}} v_{x_{2} y_{2}}\right)$ strongly to $G$. Then there is a subsequence, which we shall also denote by $\left(G_{n}\right)$, which converges pointwise. By making a change of variables, using the definition of $R(p)$ and applying Fatou's lemma, we have

$$
\begin{aligned}
0 & =\lim _{n \rightarrow \infty} \int\left|G_{n}\left(x_{1}, y_{1}, x_{2}, y_{2}\right)-G\left(x_{1}, y_{1}, x_{2}, y_{2}\right)\right|^{p} d v_{x_{1} y_{1}} d v_{x_{2} y_{2}} \\
& \geqq \int\left|G\left(x_{1}, y_{1}, x_{2}, y_{2}\right)-G\left(x_{1}, y_{2}, x_{2}, y_{1}\right)\right|^{p} d v_{x_{1} y_{2}} d v_{x_{2} y_{1}} .
\end{aligned}
$$

Since $v_{x_{1} y_{2}} v_{x_{2} y_{1}} \sim v_{x_{1} y_{1}} v_{x_{2} y_{2}}$, evidently $G=G^{*}$ a.e. $\left[v_{x_{1} y_{1}} v_{x_{2} y_{2}}\right]$.

Remarks. 1) The general case $(N \geqq 2)$ follows directly from Theorem A.3, either by grouping coordinates or by induction.

2) If each function in the weakly convergent sequence is a product of identical factors, this symmetry also holds for the weak limit.

3) The assumption $v_{x y} \sim \mu_{x y}$ in Theorem A.3 is required. In particular, as the following two examples demonstrate, neither the condition $\mu_{x y} \ll v_{x y}$ nor the condition $v_{x y} \ll \mu_{x y}$ is sufficient. In fact, the second example shows that the condition $v_{x y} \ll \mu_{x y}$ does not even guarantee that the product sector is strongly closed.

Example 1 (M. Aizenman). Let $I$ be the unit interval $[0,1]$ and let $d \mu_{x y}=d x d y$ be ordinary Lebesgue measure on $I^{2}$. Let $d v_{x y}$ be Lebesgue measure on $I^{2}$ plus a unit measure concentrated on the diagonal (i.e., $d v_{x y}=[1+\delta(x-y)] d x d y$ ). Consider the sequence of functions $F_{n}(x, y)=e^{i n(x-y)}$. For every $n, F_{n}$ is a product function on $I^{2}$, and yet the weak $L^{2}\left(d v_{x y}\right)$ limit is

$$
F= \begin{cases}1 & x=y \\ 0 & \text { otherwise }\end{cases}
$$

which is manifestly not a product in the $d v_{x y}$ measure.

Example 2. Again, let $d \mu_{x y}=d x d y$ be ordinary Lebesgue measure on the unit square $I^{2}$. Now choose $a \in(0,1)$ and let $S=I^{2} \backslash[0, a]^{2}$. Let $d v_{x y}=\chi_{S} d x d y$. The sequence of functions $F_{n}(x, y)=f_{n}(x) g_{n}(y)$, with

$$
\begin{aligned}
& f_{n}= \begin{cases}1 & x \in[0, a) \\
1 / n & x \in[a, 1]\end{cases} \\
& g_{n}= \begin{cases}n & y \in[0, a) \\
1 & y \in[a, 1],\end{cases}
\end{aligned}
$$


converges uniformly on $S$ to

$$
F=\left\{\begin{array}{llll}
1 & x \in[0, a) & \text { and } & y \in[a, 1] \\
1 & x \in[a, 1] & \text { and } & y \in[0, a), \\
0 & x \in[a, 1] & \text { and } & y \in[a, 1]
\end{array}\right.
$$

which cannot be expressed as a product.

Next, we establish a set of sufficient conditions for convergence of product functions of the form $F_{n}=\prod_{i}\left[f_{n}\left(x_{i}\right)\right]^{q_{2}}$.

Theorem A.4. Let $\langle\Lambda, d \mu\rangle$ be a $\sigma$-finite measure space. Let $\left(q_{1}, \ldots, q_{n}\right)$ be a set of distinct nonzero constants. Suppose $\left(f_{n}: \Lambda \rightarrow \mathbb{R}\right)$ is a sequence of strictly positive functions such that

$$
\prod_{i=1}^{N}\left[f_{n}\left(x_{i}\right)\right]^{q_{2}} \rightarrow F\left(x_{1}, \ldots, x_{N}\right)<\infty \quad \text { pointwise a.e. on } \Lambda^{N} .
$$

If there exists a set $A \subset \Lambda$ with $\mu(A)>0$ such that $F>0$ a.e. on $A^{N}$, then there is a measurable function $f: \Lambda \rightarrow \mathbb{R}$ such that $F=\prod_{i=1}^{N}\left[f\left(x_{i}\right)\right]^{q_{i}}$ a.e. on $\Lambda^{N}$.

Proof. Without loss of generality, we may assume $q_{1}>0$.

Choose $\tilde{x}_{2}, \ldots, \tilde{x}_{N} \in A$, so that

$$
\left[f_{n}(y)\right]^{q_{1}} \prod_{i=2}^{N}\left[f_{n}\left(\tilde{x}_{i}\right)\right]^{q_{2}} \rightarrow F\left(y, \tilde{x}_{2}, \ldots, \tilde{x}_{N}\right)<\infty
$$

for a.e. $y \in \Lambda$ and $F\left(y, \tilde{x}_{2}, \ldots, \tilde{x}_{N}\right)>0$ for a.e. $y \in A$. Since $q_{1}>0$, we may define $F\left(y, \tilde{x}_{2}, \ldots, \tilde{x}_{N}\right)=[g(y)]^{q_{1}}$ for a.e. $y \in \Lambda$.

Let $B=\{y \in \Lambda \mid g(y)>0\} \supset A$. For a.e. $y_{1}, \ldots, y_{N} \in B$

$$
C_{n}^{Q} \prod_{i=1}^{N}\left[f_{n}\left(y_{i}\right)\right]^{q_{i}} \rightarrow \prod_{i=1}^{N}\left[g\left(y_{i}\right)\right]^{q_{i}},
$$

where $C_{n}=\prod_{i=2}^{N}\left[f_{n}\left(\tilde{x}_{i}\right)\right]^{q_{i} / q_{1}}$ and $Q=\sum_{i=1}^{N} q_{i}$. Taking $y_{1}, \ldots, y_{N} \in A$, Eq. (A-7) implies that $\lim _{n \rightarrow \infty} C_{n}^{Q}$ exists and is strictly positive.

If all the charges $q_{i}$ are strictly positive, then (A-7) holds on $\Lambda^{N}$ and the proof is complete. Otherwise, Eq. (A-7) just establishes the desired result on $B^{N}$. However, it is easily verified that if there is even a single $q_{i}<0$, then $\mu(\Lambda \backslash B)=0$.

Remark. The assumption that there exist a diagonal set of positive measure on which $F>0$ a.e. is crucial for the above theorem; it is easy to construct counterexamples if this hypothesis is violated.

Finally, we generalize Theorem A.3 to convergence of product functions on direct sums of $L^{p}$ spaces. The desired result is a consequence of the following obvious proposition. 
Proposition A.5. Let $\langle\Lambda, \mu\rangle$ and $\langle\Lambda, v\rangle$ be $\sigma$-finite measure spaces with $v \ll \mu$. If $\left(f_{n}\right)$ is a sequence of functions converging weakly in $L^{p}(d \mu)$ and $L^{p}(d v)$ :

$$
\begin{array}{ll}
f_{n} \rightarrow f & \text { weakly in } L^{p}(d \mu) \\
f_{n} \rightarrow g & \text { weakly in } L^{p}(d v)
\end{array},
$$

then $f=g$ a.e. $[d v]$.

Proof. Let $\mathscr{B}$ be a (countable) partition of $\Lambda$ into disjoint measurable sets with $v(B)=1$ for every $B \in \mathscr{B}$. Let $A$ be the set on which (say) $f>g$. If $v(A)>0$, there must be a set $B \in \mathscr{B}$ with $v(B \cap A)>0$. Since $v \ll \mu$, there is a nonnegative $L^{1}(d \mu)$ function $\lambda$ with $\left.d v\right|_{B}=\lambda d \mu$. Let $B_{N}=\{x \in B \mid \lambda(x) \leqq N\}$. For all $1 \leqq q \leqq \infty, \lambda \chi_{B_{N}} \in L^{q}(d \mu)$ and $\chi_{B_{N}} \in L^{q}(d v)$. However, this clearly implies $\int_{A \cap B_{N}}(f-g) d v=0 \Rightarrow v\left(A \cap B_{N}\right)=0$. Letting $N$ tend to infinity, we obtain the desired contradiction.

Definition. Let $\langle\Lambda, d \mu\rangle$ be a $\sigma$-finite measure space and let $\left\langle\Lambda^{N}, d^{N} \mu\right\rangle$ denote its $N$-fold cartesian product. For each $N$, let $d \sigma_{N}$ be another $\sigma$-finite measure on $\Lambda^{N}$. Denote by $\langle\Lambda, d \Sigma\rangle$ the direct sum (over $N$ ) of the measure spaces $\left\langle\Lambda^{N}, d \sigma_{N}\right\rangle$. The generalized Fock space, $\mathbb{L}^{p}(d \Sigma)$, may be defined in the usual fashion [see Eq. (8.11)]. As in Sect. 8, we say that a function $F \in \mathbb{L}^{p}(d \Sigma)$ is a product function if $F=\left(F_{N}\right)$ is a collection of functions of the form $F_{N}=\prod_{i=1}^{N} f\left(x_{i}\right)$, with $f: \Lambda \rightarrow \mathbb{R}$. We
will denote this function by $\pi(f)$.

Theorem A.6. Let $\left(\pi\left(f_{n}\right)\right)$ be a sequence of product functions converging weakly in $\mathbb{L}^{p}(d \Sigma)$. If all of the measures $d \sigma_{N}$ satisfy $d \sigma_{N} \ll d^{N} \mu$ and at least one of the measures satisfies $d \sigma_{N} \sim d^{N} \mu$, then the weak $\mathbb{L}^{p}(d \Sigma)$ limit is of the form $\pi(f)$.

Proof. Weak $\mathbb{L}^{p}(d \Sigma)$ convergence implies that each term converges weakly in $L^{p}\left(d \sigma_{N}\right)$.

Let $d \sigma_{M}$ be the measure satisfying $d \sigma_{M} \sim d^{M} \mu$. Then by Theorem A.3, there is a measurable function $f: \Lambda \rightarrow \mathbb{R}$ such that $\prod_{i=1}^{M} f_{n}\left(x_{i}\right) \rightarrow \prod_{i=1}^{M} f\left(x_{i}\right)$ weakly in $L^{p}\left(d \sigma_{M}\right)$. It suffices to show that for all $N, \prod_{i=1}^{N} f_{n}\left(x_{i}\right) \rightarrow \prod_{i=1}^{N} f\left(x_{i}\right)$ weakly in $L^{p}\left(d \sigma_{N}\right)$.

Let $F\left(x_{1}, \ldots, x_{N}\right)$ denote the weak $L^{p}\left(d \sigma_{N}\right)$ limit of $\prod^{N} f_{n}\left(x_{i}\right)$. Then $\prod_{i=1}^{N M} f_{n}\left(x_{i}\right) \rightarrow \prod_{i=1}^{M} F\left(x_{1}^{i}, \ldots, x_{N}^{i}\right) \quad$ weakly in $L^{p}\left(\prod^{M} d \sigma_{N}\right)$, while $\prod_{i=1}^{N M} f_{n}\left(x_{i}\right) \rightarrow \prod_{i=1}^{N M} f\left(x_{i}\right)$ weakly in $L^{p}\left(\prod^{N} d \sigma_{M}\right)$. By Proposition A.5, the weak limits agree a.e. $\left[d \sigma_{N}\right]$.

Acknowledgements. It is a pleasure to thank J. Percus for bringing the inverse problem to our attention. We are deeply indebted to M. Aizenman and E. Nelson for numerous discussions on product functions and for independently suggesting the duplication of variables technique used in the proof of Theorem A.3. We should also like to thank M. Robert for a useful discussion on the physical implications of our results. 


\section{References}

1. Lieb, E.H.: Density functionals for Coulomb systems. In: Physics as Natural Philosophy: Essays in Honor of Laszlo Tisza on His 75 th Birthday, Shimony, A., Feshback, H. (eds.) Cambridge: M.I.T. Press 1982, p. 111

2. Levy, M.: Electron densities in search of Hamiltonians. Phys. Rev. 26 A, 1200 (1982)

3. Englisch, H., Englisch, R.: Hohenberg-Kohn theorem and non- $V$-representable densities. Physica A (in press)

4. Hugenholtz, N.M.: On the inverse problem in statistical mechanics. Commun. Math. Phys. 85, 27 (1982)

5. Evans, R.: The nature of the liquid-vapour interface and other topics in the statistical mechanics of non-uniform fluids. Adv. Phys. 28, 143 (1979)

6. Percus, J.K.: Equilibrium state of a classical fluid of hard rods in an external field. J. Stat. Phys. 15, $505(1976)$

7. Percus, J.K.: One-dimensional classical fluid with nearest-neighbor interaction in arbitrary external field. J. Stat. Phys. 28, 67 (1982)

8. Robledo, A., Varea, C.: On the relationship between the density functional formalism and the potential distribution theory for nonuniform fluids. J. Stat. Phys. 26, 513 (1981)

9. Mazur, S.: Über die kleinste konvexe Menge, die eine gegebene kompakte Menge enthält. Studia Math. 2, 7 (1930)

10. Ruelle, D.: Statistical mechanics. Reading, MA.: W. A. Benjamin, Inc. 1969

Communicated by A. Jaffe

Received July 29, 1983 
